Article

\title{
Global Mittag-Leffler Stability and Stabilization Analysis of Fractional-Order Quaternion-Valued Memristive Neural Networks
}

\author{
Grienggrai Rajchakit ${ }^{1, *}$, , Pharunyou Chanthorn ${ }^{2}$, Pramet Kaewmesri $^{3}$, \\ Ramalingam Sriraman ${ }^{4}$ and Chee Peng Lim ${ }^{5}$ \\ 1 Department of Mathematics, Faculty of Science, Maejo University, Chiang Mai 50290, Thailand \\ 2 Research Center in Mathematics and Applied Mathematics, Department of Mathematics, Faculty of Science, \\ Chiang Mai University, Chiang Mai 50200, Thailand; pharunyou.chanthorn@cmu.ac.th \\ 3 Department of Mathematics, Faculty of Science, King Mongkut's University of Technology Thonburi \\ (KMUTT), 126 Pracha-Uthit Road, Bang mod, Thung Khru 10140, Thailand; prametkae1990@gmail.com \\ 4 Vel Tech High Tech Dr.Rangarajan Dr.Sakunthala Engineering College, Avadi, Tamil Nadu-600 062, India; \\ sriraman225@gmail.com \\ 5 Institute for Intelligent Systems Research and Innovation, Deakin University, \\ Waurn Ponds, VIC 3216, Australia; chee.lim@deakin.edu.au \\ * Correspondence: kreangkri@mju.ac.th
}

Received: 13 February 2020; Accepted: 11 March 2020; Published: 14 March 2020

check for updates

\begin{abstract}
This paper studies the global Mittag-Leffler stability and stabilization analysis of fractional-order quaternion-valued memristive neural networks (FOQVMNNs). The state feedback stabilizing control law is designed in order to stabilize the considered problem. Based on the non-commutativity of quaternion multiplication, the original fractional-order quaternion-valued systems is divided into four fractional-order real-valued systems. By using the method of Lyapunov fractional-order derivative, fractional-order differential inclusions, set-valued maps, several global Mittag-Leffler stability and stabilization conditions of considered FOQVMNNs are established. Two numerical examples are provided to illustrate the usefulness of our analytical results.
\end{abstract}

Keywords: stability; stabilization; memristor; fractional calculus; quaternion-valued neural networks

\section{Introduction}

Memristor is regarded as the fourth basic circuit element, which was first proposed by Chua [1] In 2008, the first practical memristor device was invented by the HP company [2]. Recently, the memristor device has developed a large potential application due to its role in demonstrating the relationship between the magnetic flow and electric charge [3,4]. The memristor system has significant potential for synaptic use and for simulating the human brain, which can be used in artificial neural network hardware and software. One of these applications is the memristive neural networks (MNNs), which is formulated by introducing a memristor into the connection weights [5-8]. Until now, many efforts have been made to develop the dynamical behaviors of MNNs. The dynamics for various MNNs, including boundedness, stability, Mittag-Leffler stability, stabilization, synchronization, and global exponential stability, have been frequently studied in recent years [9-12].

In artificial intelligence science, neural networks (NNs) have been considered as the most important nonlinear model for their successful applications in signal processing, pattern recognition, optimization, and other engineering fields $[13,14]$. On the other side, real-valued neural networks (RVNNs) and complex-valued neural networks (CVNNs) have been successfully applied in modeling, control, associative memory, and image recognition $[15,16]$. Recently, compared to RVNNs, the CVNNs 
have shown the best performance in solving problems. For example, when encountering the problem of detection of symmetry and XOR problem, RVNNs may become helpless, while this problem can be resolved ideally with CVNNs $[17,18]$. Therefore, the occurrence of CVNNs has attracted a lot of interest from various aspects. Until now, many efforts have been made to develop the dynamical behaviors of CVNNs and numerous achievements have sprung up [12,16,19-21]. For example, the problems of global stability, global Lagrange stability, global exponential stability, passivity, and finite-time stability for CVNNs have been studied $[12,16,20,21]$.

On the other hand, as the most generalization of the RVNNs and CVNNs, QVNNs ones have been obtained, with the signals states, connection weights, and activation functions defined on the quaternion-valued [22]. In practice, the main advantage of using quaternion is that it can view and operate three-dimensional or four-dimensional vectors as a single entity that significantly reduces computational complexity in multidimensional problems and by employing quaternion variables can achieve efficient information processing directly [23-25]. Therefore, QVNNs have been successfully implemented in body images, attitude control of satellites, computer graphics, $3 D$ wind forecasting, 4D signals, color-face recognition, and vector sensor processing [26-33]. The problem of global $\mu$ stability for QVNNs with mixed time delays was studied in [22]. In [26], based on the non-commutativity of quaternion multiplication, global exponential stability for QVNNs was analyzed by separating QVNNs into four RVNNs. In [27], leakage delay-dependent synchronization conditions for fractional-order QVNNs with discrete delays have been studied. Based on the parameter uncertainties, sufficient conditions are obtained for stability and synchronization of fractional-order memristive QVNNs [30]. The problem of global Mittag-Leffler stability and synchronization for fractional-order QVNNs was studied in [31]. The problem of exponential input-to-state stability of QVNNs with time delay was studied in [32].

On the other hand, the fractional calculus has been obtained as an extension of the integer-order calculus and has received growing research attention in the fields of engineering and physics [34,35]. In addition, fractional-order derivatives have many advantages compared to integer order calculus in defining an infinite memory and inherited properties [36]. Therefore, the use of fractional-order calculus in NN models could describe better dynamic behavior, and many excellent results on fractional-order NNs have been published, such as the problem of global robust synchronization, Mittag-Leffler synchronization, quasi-pinning synchronization, and finite-time Mittag-Leffler stability [37-40]. To the best of our knowledge, the current results of this paper have not yet been proposed for FOQVMNNs. This motivates us to this current research.

Motivated by the above debates, our paper focuses primarily on analyzing the global Mittag-Leffler stability and stabilization of FOQVMNNs. On the basis of non-commutative quaternion multiplication of the Hamilton principle, the considered FOQVMNNs have been divided into four RVNNs. By use of the homeomorphism principle, Lyapunov fractional-order derivative, set-valued maps, and some analytical methods, new sufficient conditions for global Mittag-Leffler stability and stabilization of FOQVMNNs are derived. Two numerical examples are provided to illustrate our analytical results. The organization of the paper is as follows: the problem model is formally defined in the next section. The main results are described in Section 3. Section 4 yields numerical examples with their simulations. Conclusions are declared in Section 5.

\section{Preliminaries and Problem Statement}

\subsection{Notations}

$\mathcal{D}^{\sigma}$ denote $\sigma$-order fractional derivative operator. $\mathbb{R}, \mathbb{C}$, and $\mathbb{Q}$ stand for the real field, the complex field and the skew field of quaternion, respectively. $\mathbb{R}^{n}, \mathbb{C}^{n}$, and $\mathbb{Q}^{n}$ stands for $n$-dimension vector whose elements are $\mathbb{R}, \mathbb{C}$, and $\mathbb{Q}$, respectively. $\mathbb{R}^{n \times n}, \mathbb{C}^{n \times n}$ and $\mathbb{Q}^{n \times n}$ represents $n \times n$ matrices with entries from $\mathbb{R}, \mathbb{C}$ and $\mathbb{Q}$, respectively. The quaternion-valued function is denoted by $z=\left\{z^{(R)}+i z^{(I)}+j z^{(J)}+k z^{(K)} \mid z^{(R)}, z^{(I)}, z^{(J)}, z^{(K)} \in \mathbb{R}\right\}$, where $i, j, k$ are standard imaginary 
units satisfying the Hamilton rules $i j k=i^{2}=j^{2}=k^{2}=-1, i j=k=-j i, j k=i=-k j$, $k i=j=-i k$. The quaternion multiplication is non-commutative under the above Hamilton law. For any $z(t) \in \mathbb{Q}$, the modulus of $z$ is defined as $|z|=\sqrt{z z^{*}}=\sqrt{\left(z^{(R)}\right)^{2}+\left(z^{(I)}\right)^{2}+\left(z^{(J)}\right)^{2}+\left(z^{(K)}\right)^{2}}$, where $z^{*}=z^{R}(t)-i z^{I}(t)-j z^{J}(t)-k z^{K}(t)$ represents the conjugate transpose of $z$. The norm of $z$ is defined as $\|z\|=\sqrt{\sum_{x=1}^{n}\left(z^{(R)}\right)^{2}+\sum_{x=1}^{n}\left(z^{(I)}\right)^{2}+\sum_{x=1}^{n}\left(z^{(J)}\right)^{2}+\sum_{x=1}^{n}\left(z^{(K)}\right)^{2}}, N=1,2, \ldots, n$. The operation of addition and subtraction in quaternion field are similar to those in complex numbers. For the objective of simpleness, the following notations are adopted in the resulting parts:

$$
\begin{aligned}
& \left|\mathscr{G}_{y}^{R}\right|:=\left|g_{y}^{R}\left(a_{y}(t), b_{y}(t), c_{y}(t), d_{y}(t)\right)\right|, \\
& \left|\mathscr{G}_{y}^{I}\right|:=\left|g_{y}^{I}\left(a_{y}(t), b_{y}(t), c_{y}(t), d_{y}(t)\right)\right|, \\
& \left|\mathscr{G}_{y}^{J}\right|:=\left|g_{y}^{J}\left(a_{y}(t), b_{y}(t), c_{y}(t), d_{y}(t)\right)\right|, \\
& \left|\mathscr{G}_{y}^{K}\right|:=\left|g_{y}^{K}\left(a_{y}(t), b_{y}(t), c_{y}(t), d_{y}(t)\right)\right|, \\
& \left(\mathscr{G}_{y}^{*}\right):=\left(\wp_{y}^{R R}\left|a_{y}(t)\right|+\wp_{y}^{R I}\left|b_{y}(t)\right|+\wp_{y}^{R J}\left|c_{y}(t)\right|+\wp_{y}^{R K}\left|d_{y}(t)\right|\right), \\
& \left(\mathscr{G}_{y}^{* *}\right):=\left(\wp_{y}^{I R}\left|a_{y}(t)\right|+\wp_{y}^{I I}\left|b_{y}(t)\right|+\wp_{y}^{I J}\left|c_{y}(t)\right|+\wp_{y}^{I K}\left|d_{y}(t)\right|\right), \\
& \left(\mathscr{G}_{y}^{* * *}\right):=\left(\wp_{y}^{J R}\left|a_{y}(t)\right|+\wp_{y}^{J I}\left|b_{y}(t)\right|+\wp_{y}^{J J}\left|c_{y}(t)\right|+\wp_{y}^{J K}\left|d_{y}(t)\right|\right), \\
& \left(\mathscr{G}_{y}^{* * * *}\right):=\left(\wp_{y}^{K R}\left|a_{y}(t)\right|+\wp_{y}^{K I}\left|b_{y}(t)\right|+\wp_{y}^{K J}\left|c_{y}(t)\right|+\wp_{y}^{K K}\left|d_{y}(t)\right|\right), \\
& \left|a_{x}\right|^{*}\left|a_{y}\right|:=\left|a_{x}(t)\right|^{\varsigma-1}\left|a_{y}(t)\right|, \\
& \left|a_{x}\right|^{*}\left|b_{y}\right|:=\left|a_{x}(t)\right|^{\varsigma-1}\left|b_{y}(t)\right| \text {, } \\
& \left|a_{x}\right|^{*}\left|c_{y}\right|:=\left|a_{x}(t)\right|^{\varsigma-1}\left|c_{y}(t)\right|, \\
& \left|a_{x}\right|^{*}\left|d_{y}\right|:=\left|a_{x}(t)\right|^{\zeta-1}\left|d_{y}(t)\right|, \\
& \left|b_{x}\right|^{*}\left|a_{y}\right|:=\left|b_{x}(t)\right|^{\varsigma-1}\left|a_{y}(t)\right|, \\
& \left|b_{x}\right|^{*}\left|b_{y}\right|:=\left|b_{x}(t)\right|^{\varsigma-1}\left|b_{y}(t)\right|, \\
& \left|b_{x}\right|^{*}\left|c_{y}\right|:=\left|b_{x}(t)\right|^{\varsigma-1}\left|c_{y}(t)\right|, \\
& \left|b_{x}\right|^{*}\left|d_{y}\right|:=\left|b_{x}(t)\right|^{\zeta-1}\left|d_{y}(t)\right|, \\
& \left|c_{x}\right|^{*}\left|a_{y}\right|:=\left|c_{x}(t)\right|^{\varsigma-1}\left|a_{y}(t)\right|, \\
& \left|c_{x}\right|^{*}\left|b_{y}\right|:=\left|c_{x}(t)\right|^{\varsigma-1}\left|b_{y}(t)\right|, \\
& \left|c_{x}\right|^{*}\left|c_{y}\right|:=\left|c_{x}(t)\right|^{\varsigma-1}\left|c_{y}(t)\right|, \\
& \left|c_{x}\right|^{*}\left|d_{y}\right|:=\left|c_{x}(t)\right|^{\varsigma-1}\left|d_{y}(t)\right|, \\
& \left|d_{x}\right|^{*}\left|a_{y}\right|:=\left|d_{x}(t)\right|^{\zeta-1}\left|a_{y}(t)\right|, \\
& \left|d_{x}\right|^{*}\left|b_{y}\right|:=\left|d_{x}(t)\right|^{\zeta-1}\left|b_{y}(t)\right|, \\
& \left|d_{x}\right|^{*}\left|c_{y}\right|:=\left|d_{x}(t)\right|^{\zeta-1}\left|c_{y}(t)\right|, \\
& \left|d_{x}\right|^{*}\left|d_{y}\right|:=\left|d_{x}(t)\right|^{\varsigma-1}\left|d_{y}(t)\right|, \\
& \left|a^{*} a\right|_{y}:=\frac{\varsigma-1}{\varsigma}\left|a_{x}(t)\right|^{\varsigma} v_{1}+\frac{1}{\zeta}\left|a_{y}(t)\right|^{\varsigma} \frac{1}{v_{1}^{\varsigma-1}}, \\
& \left|a^{*} b\right|_{y}:=\frac{\varsigma-1}{\varsigma}\left|a_{x}(t)\right|^{\varsigma} v_{2}+\frac{1}{\varsigma}\left|b_{y}(t)\right|^{\varsigma} \frac{1}{v_{2}^{\varsigma-1}}, \\
& \left|a^{*} c\right|_{y}:=\frac{\varsigma-1}{\varsigma}\left|a_{x}(t)\right|^{\varsigma} v_{3}+\frac{1}{\varsigma}\left|c_{y}(t)\right|^{\varsigma} \frac{1}{v_{3}^{\varsigma-1}}, \\
& \left|a^{*} d\right|_{y}:=\frac{\varsigma-1}{\varsigma}\left|a_{x}(t)\right|^{\varsigma} v_{4}+\frac{1}{\varsigma}\left|d_{y}(t)\right|^{\varsigma} \frac{1}{v_{4}^{\varsigma-1}}, \\
& \left|b^{*} a\right|_{y}:=\frac{\varsigma-1}{\varsigma}\left|b_{x}(t)\right|^{\varsigma} v_{5}+\frac{1}{\varsigma}\left|a_{y}(t)\right|^{\varsigma} \frac{1}{v_{5}^{\zeta-1}}, \\
& \left|b^{*} b\right|_{y}:=\frac{\varsigma-1}{\varsigma}\left|b_{x}(t)\right|^{\varsigma} v_{6}+\frac{1}{\zeta}\left|b_{y}(t)\right|^{\varsigma} \frac{1}{v_{6}^{\varsigma-1}}, \\
& \left|b^{*} c\right|_{y}:=\frac{\varsigma-1}{\varsigma}\left|b_{x}(t)\right|^{\varsigma} v_{7}+\frac{1}{\varsigma}\left|c_{y}(t)\right|^{\varsigma} \frac{1}{v_{7}^{\varsigma-1}}, \\
& \left|b^{*} d\right|_{y}:=\frac{\varsigma-1}{\varsigma}\left|b_{x}(t)\right|^{\varsigma} v_{8}+\frac{1}{\varsigma}\left|d_{y}(t)\right|^{\varsigma} \frac{1}{v_{8}^{\varsigma-1}}, \\
& \left|c^{*} a\right|_{y}:=\frac{\varsigma-1}{\varsigma}\left|c_{x}(t)\right|^{\varsigma} v_{9}+\frac{1}{\varsigma}\left|a_{y}(t)\right|^{\varsigma} \frac{1}{v_{9}^{\zeta-1}},
\end{aligned}
$$




$$
\begin{aligned}
& \left|c^{*} b\right|_{y}:=\frac{\varsigma-1}{\varsigma}\left|c_{x}(t)\right|^{\varsigma} v_{10}+\frac{1}{\varsigma}\left|b_{y}(t)\right|^{\varsigma} \frac{1}{v_{10}^{\varsigma-1}}, \\
& \left|c^{*} c\right|_{y}:=\frac{\varsigma-1}{\varsigma}\left|c_{x}(t)\right|^{\varsigma} v_{11}+\frac{1}{\varsigma}\left|c_{y}(t)\right|^{\varsigma} \frac{1}{v_{11}^{\varsigma-1}}, \\
& \left|c^{*} d\right|_{y}:=\frac{\varsigma-1}{\varsigma}\left|c_{x}(t)\right|^{\varsigma} v_{12}+\frac{1}{\varsigma}\left|d_{y}(t)\right|^{\varsigma} \frac{1}{v_{12}^{\zeta-1}}, \\
& \left|d^{*} a\right|_{y}:=\frac{\varsigma-1}{\varsigma}\left|d_{x}(t)\right|^{\varsigma} v_{13}+\frac{1}{\zeta}\left|a_{y}(t)\right|^{\varsigma} \frac{1}{v_{13}^{\varsigma-1}}, \\
& \left|d^{*} b\right|_{y}:=\frac{\varsigma-1}{\varsigma}\left|d_{x}(t)\right|^{\varsigma} v_{14}+\frac{1}{\varsigma}\left|b_{y}(t)\right|^{\varsigma} \frac{1}{v_{14}^{\varsigma-1}}, \\
& \left|d^{*} c\right|_{y}:=\frac{\varsigma-1}{\varsigma}\left|d_{x}(t)\right|^{\varsigma} v_{15}+\frac{1}{\zeta}\left|c_{y}(t)\right|^{\varsigma} \frac{1}{v_{15}^{\zeta-1}}, \\
& \left|d^{*} d\right|_{y}:=\frac{\varsigma-1}{\varsigma}\left|d_{x}(t)\right|^{\varsigma} v_{16}+\frac{1}{\varsigma}\left|d_{y}(t)\right|^{\varsigma} \frac{1}{v_{16}^{\varsigma-1}} .
\end{aligned}
$$

\subsection{Caputo Fractional-Order Derivative}

We give the definition of Euler's gamma function $\Gamma(s)$ as

$$
\Gamma(s)=\int_{0}^{+\infty} t^{\mathfrak{r}-1} \exp (-t) d t .
$$

Definition 1 ([11]). The fractional-order of Caputo derivative of order $\sigma$ for a function $\bar{w}(t) \in C^{n}\left(\left[t_{0},+\infty\right), \mathbb{R}\right)$ is defined as

$$
{ }_{t_{0}}^{C} \mathcal{D}_{t}^{\sigma} \bar{w}(t)=\frac{1}{\Gamma(n-\sigma)} \int_{t_{0}}^{t} \frac{\bar{w}^{(n)}(\ell)}{(t-\ell)^{\sigma-n+1}} d \ell,
$$

where $t \geq t_{0}$ and $n$ is the positive integer such that $n-1<\sigma<n$, and $n \in \mathbb{Z}^{+} . \Gamma(\cdot)$ is gamma function.

Definition 2 ([11]). The fractional-order of integral order $\sigma \in(0,1)$ for a integral function $\bar{w} \in$ $C^{n}([0,+\infty), \mathbb{R})$ is defined as

$$
{ }_{t_{0}} \mathcal{D}_{t}^{-\sigma} \bar{w}(t)=\frac{1}{\Gamma(\sigma)} \int_{0}^{t} \frac{\bar{w}(\ell)}{(t-\ell)^{1-\sigma}} d \ell, t>0, \sigma>0 .
$$

\subsection{Problem Statement}

In this section, we consider a class of FOQVMNNs as follows:

$$
{ }^{C} \mathcal{D}_{0, t}^{\sigma} z_{x}(t)=-\mathbf{a}_{x} z_{x}(t)+\sum_{y=1}^{n} \mathbf{b}_{x y}\left(z_{x}(t)\right) f_{y}\left(z_{y}(t)\right)+I_{x}, t>0, x \in N,
$$

where $0<\sigma<1, z_{x}(t)$ is the state variable of the $x^{t h}$ neuron (the voltage of capacitor), $a_{x}>0$ is the self-regulating parameters of the neurons, $\mathbf{b}_{x y}\left(z_{x}(t)\right)$ is the memristive connective weights, which denote the neuron interconnection matrix. $f_{y}(\cdot): \mathbb{Q} \rightarrow \mathbb{Q}$ is the bounded feedback function between the $y^{\text {th }}$ dimension of the memristor. $I_{x}$ represents the external input. According to the feature of memristor, denote

$$
\mathbf{b}_{x y}\left(z_{x}(t)\right)= \begin{cases}\dot{\mathbf{b}}_{x y}, & \left|z_{x}(t)\right|<\mathscr{T}_{y} \\ \grave{\mathbf{b}}_{x y}, & \left|z_{x}(t)\right|>\mathscr{T}_{y}\end{cases}
$$

where $\mathbf{b}_{x y}\left( \pm \mathscr{T}_{y}\right)=\mathfrak{\mathbf { b }}_{x y}$ or $\grave{\mathbf{b}}_{x y}, \boldsymbol{\mathbf { b }}_{x y}, \grave{\mathbf{b}}_{x y}$ are constants and $\mathscr{T}_{y}>0$ is the switching jumps. 
It is obvious that $\mathbf{b}_{x y}\left(z_{x}(t)\right)$ in NN (1) is discontinuous. Similar to the work [5], by applying the theories of set-valued maps and differential inclusions, NN (1) can be written as the following differential inclusion:

$$
{ }^{C} \mathcal{D}_{0, t}^{\sigma} z_{x}(t) \in-\mathbf{a}_{x} z_{x}(t)+\sum_{y=1}^{n} c o\left[\mathbf{b}_{x y}\left(z_{x}(t)\right)\right] f_{y}\left(z_{y}(t)\right)+I_{x}, t>0, x \in N,
$$

where

$$
\operatorname{co}\left[\mathbf{b}_{x y}\left(z_{x}(t)\right)\right]=\left\{\begin{array}{l}
\hat{\mathbf{b}}_{x y},\left|z_{x}(t)\right|<\mathscr{T}_{y} \\
\operatorname{co}\left\{\hat{\mathbf{b}}_{x y}, \grave{\mathbf{b}}_{x y}\right\}, \quad\left|z_{x}(t)\right|=\mathscr{T}_{y}, \\
\grave{\mathbf{b}}_{x y},\left|z_{x}(t)\right|>\mathscr{T}_{y}
\end{array}\right.
$$

and $\operatorname{co}\left\{\hat{\mathbf{b}}_{x y}, \grave{\mathbf{b}}_{x y}\right\}=\left[\underline{\mathbf{b}}_{x y}, \overline{\mathbf{b}}_{x y}\right], \underline{\mathbf{b}}_{x y}=\min \left\{\hat{\mathbf{b}}_{x y}, \grave{\mathbf{b}}_{x y}\right\}, \overline{\mathbf{b}}_{x y}=\max \left\{\hat{\mathbf{b}}_{x y}, \grave{\mathbf{b}}_{x y}\right\}, \tilde{\alpha}_{x y}=\max \left\{\left|\hat{\mathbf{b}}_{x y}\right|,\left|\grave{\mathbf{b}}_{x y}\right|\right\}$.

Remark 1. When the NNs (2) can be divided into real and imaginary parts, by applying the theories of set-valued maps and differential inclusions, we have co $\left\{\mathbf{b}_{x y}^{R}, \grave{\mathbf{b}}_{x y}^{R}\right\}=\left[\underline{\mathbf{b}}_{x y}^{R}, \overline{\mathbf{b}}_{x y}^{R}\right], \underline{\mathbf{b}}_{x y}^{R}=\min \left\{\mathbf{b}_{x y}^{R}, \grave{\mathbf{b}}_{x y}^{R}\right\}$, $\overline{\mathbf{b}}_{x y}^{R}=\max \left\{\hat{\mathbf{b}}_{x y}^{R}, \grave{\mathbf{b}}_{x y}^{R}\right\}, \tilde{\alpha}_{x y}^{R}=\max \left\{\left|\hat{\mathbf{b}}_{x y}^{R}\right|,\left|\grave{\mathbf{b}}_{x y}^{R}\right|\right\}, \operatorname{co}\left\{\hat{\mathbf{b}}_{x y}^{I}, \grave{\mathbf{b}}_{x y}^{I}\right\}=\left[\underline{\mathbf{b}}_{x y}^{I}, \overline{\mathbf{b}}_{x y}^{I}\right], \underline{\mathbf{b}}_{x y}^{I}=\min \left\{\hat{\mathbf{b}}_{x y}^{I}, \grave{\mathbf{b}}_{x y}^{I}\right\}$, $\overline{\mathbf{b}}_{x y}^{I}=\max \left\{\hat{\mathbf{b}}_{x y}^{I}, \grave{\mathbf{b}}_{x y}^{I}\right\}, \tilde{\alpha}_{x y}^{I}=\max \left\{\left|\hat{\mathbf{b}}_{x y}^{I}\right|,\left|\grave{\mathbf{b}}_{x y}^{I}\right|\right\}, \operatorname{co}\left\{\hat{\mathbf{b}}_{x y}^{J}, \grave{\mathbf{b}}_{x y}^{J}\right\}=\left[\underline{\mathbf{b}}_{x y}^{J}, \overline{\mathbf{b}}_{x y}^{J}\right], \underline{\mathbf{b}}_{x y}^{J}=\min \left\{\hat{\mathbf{b}}_{x y}^{J}, \grave{\mathbf{b}}_{x y}^{J}\right\}$, $\overline{\mathbf{b}}_{x y}^{J}=\max \left\{\hat{\mathbf{b}}_{x y}^{J}, \grave{\mathbf{b}}_{x y}^{J}\right\}, \tilde{\alpha}_{x y}^{J}=\max \left\{\left|\grave{\mathbf{b}}_{x y}^{J}\right|,\left|\grave{\mathbf{b}}_{x y}^{J}\right|\right\}, \operatorname{co}\left\{\hat{\mathbf{b}}_{x y}^{K}, \grave{\mathbf{b}}_{x y}^{K}\right\}=\left[\underline{\mathbf{b}}_{x y}^{K}, \overline{\mathbf{b}}_{x y}^{K}\right], \underline{\mathbf{b}}_{x y}^{K}=\min \left\{\hat{\mathbf{b}}_{x y}^{K}, \grave{\mathbf{b}}_{x y}^{K}\right\}$, $\overline{\mathbf{b}}_{x y}^{K}=\max \left\{\hat{\mathbf{b}}_{x y}^{K}, \grave{\mathbf{b}}_{x y}^{K}\right\}, \tilde{\alpha}_{x y}^{K}=\max \left\{\left|\hat{\mathbf{b}}_{x y}^{K}\right|,\left|\grave{\mathbf{b}}_{x y}^{K}\right|\right\}$.

Based on the measurable selection theorem in [5], there exist measurable functions $\alpha_{x y}\left(z_{x}(t)\right) \in$ $c o\left[\mathbf{b}_{x y}\left(z_{x}(t)\right)\right]$ for $x, y \in N$, such that

$$
{ }^{C} \mathcal{D}_{0, t}^{\sigma} z_{x}(t)=-\mathbf{a}_{x} z_{x}(t)+\sum_{y=1}^{n} \alpha_{x y}\left(z_{x}(t)\right) f_{y}\left(z_{y}(t)\right)+I_{x}, t>0, x \in N .
$$

The initial condition of (3) is defined as $z_{x}(0)=z_{0} \in \mathbb{Q}$, where $z_{0}=z^{R}(0)+i z^{I}(0)+j z^{J}(0)+k z^{K}(0)$. A1: In the quaternion domain, the function $f_{y}(\cdot), y \in N$ is bounded, and $f_{y}\left( \pm \mathscr{T}_{y}\right)=0$ satisfies the Lipschitz condition with Lipschitz constant $l_{y}>0, y \in N$,

$$
\left|f_{y}\left(z_{1}\right)-f_{y}\left(z_{2}\right)\right| \leq l_{y}\left|z_{1}-z_{2}\right|, \forall z_{1}, z_{2} \in \mathbb{Q}, y \in N \text {. }
$$

A2: A constant vector $z^{*}(t)=\left[z_{1}^{*}(t), \ldots, z_{n}^{*}(t)\right]^{T} \in \mathbb{Q}^{n}$ is called an equilibrium point of NNs (3) if

$$
0=-a_{x} z_{x}^{*}(t)+\sum_{y=1}^{n} \alpha_{x y}\left(z_{x}^{*}(t)\right) f_{y}\left(z_{y}^{*}(t)\right)+I_{x}, x \in N .
$$

\subsection{Preliminaries}

To derive the main results, the following definitions and lemmas are needed.

Definition 3 ([41]). The Mittag-Leffler function with one parameter is defined as

$$
\mathscr{E}_{\sigma}(z)=\sum_{x=0}^{\infty} \frac{z^{x}}{\Gamma(x \sigma+1)}
$$


where $\sigma>0$. The Mittag-Leffler function with one parameter is defined as

$$
\mathscr{E}_{\sigma, \rho}(z)=\sum_{x=0}^{\infty} \frac{z^{x}}{\Gamma(x \sigma+\rho)}
$$

where $\sigma>0, \rho>0$. When $\rho=1$, one has $\mathscr{E}_{\sigma, 1}=\mathscr{E}(z)$ and, when $\sigma=1, \rho=1$, one further has $\mathscr{E}_{1,1}=e^{z}$.

Definition 4 ([41]). The Laplace transform of Mittag-Leffler function with two parameters is

$$
\mathcal{L}\left\{t^{\rho-1} \mathscr{E}_{\sigma, \rho}\left(-\lambda t^{\sigma}\right)\right\}=\frac{\tau^{\sigma-\rho}}{\tau^{\sigma}+\lambda}, \operatorname{Re}(\tau)>|\lambda|^{\frac{1}{\sigma}}
$$

where $t$ and $\tau$ are, respectively, the variables in the Laplace domain and time domain, $\lambda$ is real number, $\mathcal{L}(\cdot)$ represents the Laplace transform.

Lemma 1 ([42]). Let $\eta_{1}>0, \eta_{2}>0, \eta_{3}>1, \eta_{4}>1$, and $\frac{1}{\eta_{3}}+\frac{1}{\eta_{4}}=1$. Then, for any $\varsigma>0$, we have

$$
\eta_{1} \eta_{2} \leq \frac{1}{\eta_{3}}\left(\eta_{1} \varsigma\right)^{\eta_{3}}+\frac{1}{\eta_{4}}\left(\eta_{2} \frac{1}{\varsigma}\right)^{\eta_{4}}
$$

the equality holds iff $\left(\eta_{1} \varsigma\right)^{\eta_{3}}=\left(\eta_{2} \frac{1}{\zeta}\right)^{\eta_{4}}$.

Lemma 2 ([42]). Let $\mathscr{F}(t)$ be a continuous function on $[0,+\infty)$ satisfying

$$
{ }^{C} \mathcal{D}_{0, t}^{\sigma} \mathscr{F}(t) \leq-\eta_{1} \mathscr{F}(t)+\eta_{2}, t \geq 0 .
$$

for constant $\eta_{1}>0, \eta_{2} \geq 0$, then

$$
\left.\mathscr{F}(t) \leq \mathscr{F}(0) \mathscr{E}_{\sigma}\left(-k_{1} t^{\sigma}\right)+k_{2} t^{\sigma} \mathscr{E}_{\sigma, \sigma+1}\left(-k_{1} t^{\sigma}\right)\right), t \geq 0 .
$$

where $0<\sigma<1, \mathscr{E}_{\sigma, \sigma+1}(\cdot)$ and $\mathscr{E}_{\sigma}(\cdot)$ are, respectively, two-parameter Mittag-Leffler function and one-parameter Mittag-Leffler function.

Definition 5 ([41]). The zero solution of the system (3) is said to be globally Mittag-Leffler stable if there exist two positive constants $\xi$ and $\lambda$ such that, for any solutions $z(t)$ of the system (3) with different initial condition denoted by $z(0)$, one has

$$
\|z(t)\| \leq \xi\|z(0)\| \mathscr{E}_{\sigma}\left(-\lambda t^{\sigma}\right), t>0
$$

Definition 6 ([42]). The system (3) is globally Mittag-Leffler stabilizable if there exists a suitable feedback control law, such that the closed-loop system (3) is globally Mittag-Leffler-stable.

\section{Main Results}

This section will present new sufficient conditions for global Mittag-Leffler stability and stabilization of FOQVMNNs (3) under A1. In order to prove the equilibrium point $z^{*}(t)$ is global Mittag-Leffler stability by the transformation $\sim w_{x}(t)=z_{x}(t)-z^{*}(t)$, the equilibrium point for system (3) can be shifted to the origin and obtain

$$
{ }^{C} \mathcal{D}_{0, t}^{\sigma} w_{x}(t)=-\left[\mathbf{a}_{x} z_{x}(t)-\mathbf{a}_{x} z_{x}^{*}(t)\right]+\sum_{y=1}^{n}\left[\alpha_{x y}\left(z_{x}(t)\right) f_{y}\left(z_{y}(t)\right)-\alpha_{x y}\left(z_{x}^{*}(t)\right) f_{y}\left(z_{y}^{*}(t)\right)\right], t>0, x \in N,
$$


or, equivalently,

$$
{ }^{C} \mathcal{D}_{0, t}^{\sigma} w_{x}(t)=-\mathbf{a}_{x} w_{x}(t)+\sum_{y=1}^{n} \alpha_{x y} g_{y}\left(w_{y}(t)\right), t>0, x \in N
$$

where $g_{y}\left(w_{y}(t)\right)=\left(f_{y}\left(w_{y}(t)\right)+f\left(z_{y}^{*}(t)\right)\right)-f\left(z_{y}^{*}(t)\right)$.

Remark 2. It should be noted that the multiplication of quaternion does not comply with commutative law, which varies entirely from complex and real numbers. Therefore, CVNN or RVNN analysis approaches and techniques are not explicitly used to evaluate QVNNs. In order to analyze QVNNs easily, a suitable approach is to divide the QVNN into two CVNNs or four RVNNs systems based on the non-commutative quaternion multiplication.

Let $w_{x}(t)=a_{x}(t)+i b_{x}(t)+j c_{x}(t)+k d_{x}(t)$; then, the system can be expressed as follows:

$$
\left\{\begin{aligned}
&{ }^{C} \mathcal{D}_{0, t}^{\sigma} a_{x}(t)=-\mathbf{a}_{x} a_{x}(t)+\sum_{y=1}^{n} \alpha_{x y}^{R}\left(a_{x}(t)\right) g_{y}^{R}\left(a_{y}(t), b_{y}(t), c_{y}(t), d_{y}(t)\right)-\sum_{y=1}^{n} \alpha_{x y}^{I}\left(b_{x}(t)\right) g_{y}^{I}\left(a_{y}(t), b_{y}(t), c_{y}(t), d_{y}(t)\right) \\
&-\sum_{y=1}^{n} \alpha_{x y}^{I}\left(c_{x}(t)\right) g_{y}^{I}\left(a_{y}(t), b_{y}(t), c_{y}(t), d_{y}(t)\right)-\sum_{y=1}^{n} \alpha_{x y}^{K}\left(d_{x}(t)\right) g_{y}^{K}\left(a_{y}(t), b_{y}(t), c_{y}(t), d_{y}(t)\right) \\
&{ }^{C} \mathcal{D}_{0, t}^{\sigma} b_{x}(t)=-\mathbf{a}_{x} b_{x}(t)+\sum_{y=1}^{n} \alpha_{x y}^{R}\left(a_{x}(t)\right) g_{y}^{I}\left(a_{y}(t), b_{y}(t), c_{y}(t), d_{y}(t)\right)+\sum_{y=1}^{n} \alpha_{x y}^{I}\left(b_{x}(t)\right) g_{y}^{R}\left(a_{y}(t), b_{y}(t), c_{y}(t), d_{y}(t)\right) \\
&+\sum_{y=1}^{n} \alpha_{x y}^{I}\left(c_{x}(t)\right) g_{y}^{K}\left(a_{y}(t), b_{y}(t), c_{y}(t), d_{y}(t)\right)-\sum_{y=1}^{n} \alpha_{x y}^{K}\left(d_{x}(t)\right) g_{y}^{I}\left(a_{y}(t), b_{y}(t), c_{y}(t), d_{y}(t)\right) \\
&-\mathbf{a}_{x} c_{x}(t)+\sum_{y=1}^{n} \alpha_{x y}^{R}\left(a_{x}(t)\right) g_{y}^{I}\left(a_{y}(t), b_{y}(t), c_{y}(t), d_{y}(t)\right)+\sum_{y=1}^{n} \alpha_{x y}^{I}\left(c_{x}(t)\right) g_{y}^{R}\left(a_{y}(t), b_{y}(t), c_{y}(t), d_{y}(t)\right) \\
&+\sum_{y=1}^{n} \alpha_{x y}^{K}\left(d_{x}(t)\right) g_{y}^{I}\left(a_{y}(t), b_{y}(t), c_{y}(t), d_{y}(t)\right)-\sum_{y=1}^{n} \alpha_{x y}^{I}\left(b_{x}(t)\right) g_{y}^{K}\left(a_{y}(t), b_{y}(t), c_{y}(t), d_{y}(t)\right) \\
&-\mathbf{a}_{x} d_{x}(t)+\sum_{y=1}^{n} \alpha_{x y}^{R}\left(a_{x}(t)\right) g_{y}^{K}\left(a_{y}(t), b_{y}(t), c_{y}(t), d_{y}(t)\right)+\sum_{y=1}^{n} \alpha_{x y}^{I}\left(b_{x}(t)\right) g_{y}^{I}\left(a_{y}(t), b_{y}(t), c_{y}(t), d_{y}(t)\right) \\
&+\sum_{y=1}^{n} \alpha_{x y}^{K}\left(d_{x}(t)\right) g_{y}^{R}\left(a_{y}(t), b_{y}(t), c_{y}(t), d_{y}(t)\right)-\sum_{y=1}^{n} \alpha_{x y}^{I}\left(c_{x}(t)\right) g_{y}^{I}\left(a_{y}(t), b_{y}(t), c_{y}(t), d_{y}(t)\right) \\
&{ }^{C} \mathcal{D}_{0, t}^{\sigma} d_{x}(t)
\end{aligned}\right.
$$

We can rewrite $\mathrm{NN}(7)$ in the vector form as follows:

$$
\left\{\begin{aligned}
{ }^{C} \mathcal{D}_{0, t}^{\sigma} a(t)= & -\mathbf{A} a(t)+\mathbf{B}^{R}(a(t)) g^{R}(a(t), b(t), c(t), d(t))-\mathbf{B}^{I}(b(t)) g^{I}(a(t), b(t), c(t), d(t)) \\
& -\mathbf{B}^{J}(c(t)) g^{J}(a(t), b(t), c(t), d(t))-\mathbf{B}^{K}(d(t)) g^{K}(a(t), b(t), c(t), d(t)) \\
{ }^{C} \mathcal{D}_{0, t}^{\sigma} b(t)= & -\mathbf{A} b(t)+\mathbf{B}^{R}(a(t)) g^{I}(a(t), b(t), c(t), d(t))+\mathbf{B}^{I}(b(t)) g^{R}(a(t), b(t), c(t), d(t)) \\
& +\mathbf{B}^{J}(c(t)) g^{K}(a(t), b(t), c(t), d(t))-\mathbf{B}^{K}(d(t)) g^{J}(a(t), b(t), c(t), d(t)) \\
{ }^{C} \mathcal{D}_{0, t}^{\sigma} c(t)= & -\mathbf{A} c(t)+\mathbf{B}^{R}(a(t)) g^{J}(a(t), b(t), c(t), d(t))+\mathbf{B}^{J}(c(t)) g^{R}(a(t), b(t), c(t), d(t)) \\
& +\mathbf{B}^{K}(d(t)) g^{I}(a(t), b(t), c(t), d(t))-\mathbf{B}^{I}(b(t)) g^{K}(a(t), b(t), c(t), d(t)) \\
{ }^{C} \mathcal{D}_{0, t}^{\sigma} d(t)= & -\mathbf{A} d(t)+\mathbf{B}^{R}(a(t)) g^{K}(a(t), b(t), c(t), d(t))+\mathbf{B}^{I}(b(t)) g^{J}(a(t), b(t), c(t), d(t)) \\
& +\mathbf{B}^{K}(d(t)) g^{R}(a(t), b(t), c(t), d(t))-\mathbf{B}^{J}(c(t)) g^{I}(a(t), b(t), c(t), d(t))
\end{aligned}\right.
$$

where $a(t)=\left[a_{1}(t), \ldots, a_{n}(t)\right]^{T}, b(t)=\left[b_{1}(t), \ldots, b_{n}(t)\right]^{T}, c(t)=\left[c_{1}(t), \ldots, c_{n}(t)\right]^{T}, d(t)=$ $\left[d_{1}(t), \ldots, d_{n}(t)\right]^{T}, \mathbf{A}=\operatorname{diag}\left\{a_{1}, \ldots, a_{n}\right\}, \mathbf{B}^{R}(a(t))=\left(\alpha_{x y}^{R}\left(a_{x}(t)\right)\right)_{n \times n} \in \mathbb{R}^{n \times n}, \mathbf{B}^{I}(b(t))=$ $\left(\alpha_{x y}^{I}\left(b_{x}(t)\right)\right)_{n \times n} \in \mathbb{R}^{n \times n}, \mathbf{B}^{J}(c(t))=\left(\alpha_{x y}^{J}\left(c_{x}(t)\right)\right)_{n \times n} \in \mathbb{R}^{n \times n}, \mathbf{B}^{K}(d(t))=\left(\alpha_{x y}^{K}\left(d_{x}(t)\right)\right)_{n \times n} \in \mathbb{R}^{n \times n}$.

The initial condition of (8) is defined as $w_{x}(0)=w_{0} \in \mathbb{R}^{n}$, where $w_{0}=$ $\left(\left(a^{R}(0)\right)^{T},\left(b^{I}(0)\right)^{T},\left(c^{J}(0)\right)^{T},\left(d^{K}(0)\right)^{T}\right)^{T}$.

A3: For $w=\mathfrak{a}^{R}+i \mathfrak{b}^{I}+j \mathfrak{c} J+k \mathfrak{d}^{K} \in \mathbb{Q}^{n}$ with $\mathfrak{a}^{R}, \mathfrak{b}^{I}, \mathfrak{c}^{J}, \mathfrak{d}^{K} \in \mathbb{R}, g_{y}(w)$ can be expressed as

$$
g_{y}(w)=g_{y}^{R}\left(\mathfrak{a}^{R}, \mathfrak{b}^{I}, \mathfrak{c}^{J}, \mathfrak{d}^{K}\right)+i g_{y}^{I}\left(\mathfrak{a}^{R}, \mathfrak{b}^{I}, \mathfrak{c}^{J}, \mathfrak{d}^{K}\right)+j g_{y}^{I}\left(\mathfrak{a}^{R}, \mathfrak{b}^{I}, \mathfrak{c}^{J}, \mathfrak{d}^{K}\right)+k g_{y}^{K}\left(\mathfrak{a}^{R}, \mathfrak{b}^{I}, \mathfrak{c}^{J}, \mathfrak{d}^{K}\right),
$$

where $y \in N, g^{R}(., \ldots,),. g^{I}(., \ldots,),. g^{J}(., \ldots, .),. g^{K}(., \ldots, .):. \mathbb{R}^{4} \rightarrow \mathbb{R}$, the real-imaginary parts satisfies 
(1) The partial derivatives of $g_{y}(., \ldots, .$.$) with respect to the variables \mathfrak{a}^{R}, \mathfrak{b}^{I}, \mathfrak{c}^{J}, \mathfrak{d}^{K}$ exists and are continuous.

(2) All the partial derivatives are bounded, i.e., there exist positive constant numbers $\wp_{y}^{R R}, \wp_{y}^{R I}, \wp_{y}^{R J}$, $\wp_{y}^{R K}, \wp_{y}^{I R}, \wp_{y}^{I I}, \wp_{y}^{I J}, \wp_{y}^{I K}, \wp_{y}^{J R}, \wp_{y}^{I I}, \wp_{y}^{J J}, \wp_{y}^{J K}, \wp_{y}^{K R}, \wp_{y}^{K I}, \wp_{y}^{K J}, \wp_{y}^{K K}$, such that

$$
\begin{aligned}
& \left|\frac{\partial g_{y}^{R}}{\partial \mathfrak{a}^{R}}\right| \leq \wp_{y}^{R R},\left|\frac{\partial g_{y}^{R}}{\partial \mathfrak{b}^{I}}\right| \leq \wp_{y}^{R I},\left|\frac{\partial g_{y}^{R}}{\partial \mathfrak{c}^{J}}\right| \leq \wp_{y}^{R J},\left|\frac{\partial g_{y}^{R}}{\partial \mathfrak{d}^{K}}\right| \leq \wp_{y}^{R K}, \\
& \left|\frac{\partial g_{y}^{I}}{\partial \mathfrak{a}^{R}}\right| \leq \wp_{y}^{I R},\left|\frac{\partial g_{y}^{I}}{\partial \mathfrak{b}^{I}}\right| \leq \wp_{y}^{I I},\left|\frac{\partial g_{y}^{I}}{\partial \mathfrak{c}^{J}}\right| \leq \wp_{y}^{I J},\left|\frac{\partial g_{y}^{I}}{\partial \mathfrak{d}^{K}}\right| \leq \wp_{y}^{I K}, \\
& \left|\frac{\partial g_{y}^{J}}{\partial \mathfrak{a}^{R}}\right| \leq \wp_{y}^{J R},\left|\frac{\partial g_{y}^{J}}{\partial \mathfrak{b}^{I}}\right| \leq \wp_{y}^{I I},\left|\frac{\partial g_{y}^{J}}{\partial \mathfrak{c}^{J}}\right| \leq \wp_{y}^{J J},\left|\frac{\partial g_{y}^{J}}{\partial \mathfrak{d}^{K}}\right| \leq \wp_{y}^{J K}, \\
& \left|\frac{\partial g_{y}^{k}}{\partial \mathfrak{a}^{R}}\right| \leq \wp_{y}^{K R},\left|\frac{\partial g_{y}^{K}}{\partial \mathfrak{b}^{I}}\right| \leq \wp_{y}^{K I},\left|\frac{\partial g_{y}^{K}}{\partial \mathfrak{c}^{J}}\right| \leq \wp_{y}^{K J},\left|\frac{\partial g_{y}^{K}}{\partial \mathfrak{d}^{K}}\right| \leq \wp_{y}^{K K} .
\end{aligned}
$$

Then, one can obtain that the following inequalities

$$
\begin{aligned}
& \left|g_{y}^{R}\left(\mathfrak{\mathfrak { a }}^{R}, \mathfrak{\mathfrak { b }}^{I}, \mathfrak{\mathfrak { c }}^{J}, \mathfrak{d}^{K}\right)-g_{y}^{R}\left(\grave{\mathfrak{a}}^{R}, \grave{\mathfrak{b}}^{I}, \grave{\mathfrak{c}}^{J}, \grave{\mathfrak{d}}^{K}\right)\right| \leq \wp_{y}^{R R}\left|\mathfrak{\mathfrak { a }}^{R}-\grave{\mathfrak{a}}^{R}\right|+\wp_{y}^{R I}\left|\mathfrak{\mathfrak { b }}^{I}-\grave{\mathfrak{b}}^{I}\right|+\wp_{y}^{R J}\left|\mathfrak{\mathfrak { c }}^{J}-\grave{\mathfrak{c}}^{J}\right|+\wp_{y}^{R K}\left|\mathfrak{\mathfrak { d }}^{K}-\grave{\mathfrak{d}}^{K}\right|, \\
& \left|g_{y}^{I}\left(\hat{\mathfrak{a}}^{R}, \mathfrak{b}^{I}, \mathfrak{\mathfrak { c }}^{J}, \mathfrak{\mathfrak { d }}^{K}\right)-g_{y}^{I}\left(\grave{\mathfrak{a}}^{R}, \mathfrak{\mathfrak { b }}^{I}, \grave{\mathfrak{c}}^{J}, \grave{\mathfrak{d}}^{K}\right)\right| \leq \wp_{y}^{I R}\left|\mathfrak{\mathfrak { a }}^{R}-\grave{\mathfrak{a}}^{R}\right|+\wp_{y}^{I I}\left|\mathfrak{\mathfrak { b }}^{I}-\grave{\mathfrak{b}}^{I}\right|+\wp_{y}^{I J}\left|\mathfrak{\mathfrak { c }}^{J}-\grave{\mathfrak{c}}^{J}\right|+\wp_{y}^{I K}\left|\mathfrak{\mathfrak { d }}^{K}-\grave{\mathfrak{d}}^{K}\right|, \\
& \left|g_{y}^{J}\left(\hat{\mathfrak{a}}^{R}, \mathfrak{\mathfrak { b }}^{I}, \hat{\mathfrak{c}}^{J}, \mathfrak{\mathfrak { d }}^{K}\right)-g_{y}^{J}\left(\grave{\mathfrak{a}}^{R}, \mathfrak{\mathfrak { b }}^{I}, \grave{\mathfrak{c}}^{J}, \grave{\mathfrak{d}}^{K}\right)\right| \leq \wp_{y}^{J R}\left|\mathfrak{\mathfrak { a }}^{R}-\grave{\mathfrak{a}}^{R}\right|+\wp_{y}^{J I}\left|\mathfrak{\mathfrak { b }}^{I}-\grave{\mathfrak{b}}^{I}\right|+\wp_{y}^{J J}\left|\mathfrak{\mathfrak { c }}^{J}-\grave{\mathfrak{c}}^{J}\right|+\wp_{y}^{J K}\left|\mathfrak{\mathfrak { d }}^{K}-\grave{\mathfrak{d}}^{K}\right|, \\
& \left|g_{y}^{K}\left(\hat{\mathfrak{a}}^{R}, \mathfrak{\mathfrak { b }}^{I}, \mathfrak{\mathfrak { c }}^{J}, \mathfrak{d}^{K}\right)-g_{y}^{K}\left(\grave{\mathfrak{a}}^{R}, \grave{\mathfrak{b}}^{I}, \grave{\mathfrak{c}}^{J}, \grave{\mathfrak{d}}^{K}\right)\right| \leq \wp_{y}^{K R}\left|\hat{\mathfrak{a}}^{R}-\grave{\mathfrak{a}}^{R}\right|+\wp_{y}^{K I}\left|\mathfrak{\mathfrak { b }}^{I}-\grave{\mathfrak{b}}^{I}\right|+\wp_{y}^{K J}\left|\mathfrak{\mathfrak { c }}^{J}-\grave{\mathfrak{c}}^{J}\right|+\wp_{y}^{K K}\left|\mathfrak{\mathfrak { d }}^{K}-\grave{\mathfrak{d}}^{K}\right|,
\end{aligned}
$$

hold for any $\mathfrak{a}^{R}, \mathfrak{b}^{I}, \mathfrak{\mathfrak { c }}^{J}, \mathfrak{d}^{K}, \grave{\mathfrak{a}}^{R}, \grave{\mathfrak{b}}^{I}, \grave{\mathfrak{c}}^{J}, \grave{\mathfrak{d}}^{K} \in \mathbb{R}$. Moreover, $g_{y}(0)=0$ for all $y \in N$.

Remark 3. Recently, several activation functions been considered to study the QVNNs $[32,33,38,40]$. There are two different approaches that have been well regarded among them. The first approach is that the activation functions are not expressed directly by dividing real and imaginary parts [33], and the second approach is that the function of activation can be expressed by dividing real and imaginary parts [40]. Accordingly, the main results of this paper will be derived by using the real-imaginary separate type activation function.

\subsection{Global Mittag-Leffler Stability}

This section describes new global Mittag-Leffler stability conditions for NNs (7), which is shown in the following Theorem 1.

Theorem 1. If there exist constants $\varsigma>1$ and $v_{\ell}>0, \ell=1,2, \ldots, 16$, such that, for $x \in N$,

$$
\left\{\begin{array}{l}
\mathbf{a}_{x} \varsigma-\sum_{y=1}^{n}\left[\tilde{\alpha}_{x y}^{R} \wp_{y}^{R R}-\tilde{\alpha}_{x y}^{I} \wp_{y}^{I R}-\tilde{\alpha}_{x y}^{J} \wp_{y}^{J R}-\tilde{\alpha}_{x y}^{K} \wp_{y}^{K R}\right](\varsigma-1) v_{1}-\sum_{y=1}^{n}\left[\tilde{\alpha}_{y x}^{R} \wp_{x}^{R R}-\tilde{\alpha}_{y x}^{I} \wp_{x}^{I R}\right. \\
\left.-\tilde{\alpha}_{y x}^{J} \wp_{x}^{J R}-\tilde{\alpha}_{y x}^{K} \wp_{x}^{K R}\right] \frac{1}{v_{1}^{S-1}}-\sum_{y=1}^{n}\left[\tilde{\alpha}_{x y}^{R} \wp_{y}^{R I}-\tilde{\alpha}_{x y}^{I} \wp_{y}^{I I}-\tilde{\alpha}_{x y}^{J} \wp_{y}^{J I}-\tilde{\alpha}_{x y}^{K} \wp_{y}^{K I}\right](\varsigma-1) v_{2} \\
-\sum_{y=1}^{n}\left[\tilde{\alpha}_{x y}^{R} \wp_{y}^{R I}-\tilde{\alpha}_{x y}^{I} \wp_{y}^{I J}-\tilde{\alpha}_{x y}^{I} \wp_{y}^{J J}-\tilde{\alpha}_{x y}^{K} \wp_{y}^{K J}\right](\varsigma-1) v_{3}-\sum_{y=1}^{n}\left[\tilde{\alpha}_{x y}^{R} \wp_{y}^{R K}-\tilde{\alpha}_{x y}^{I} \wp_{y}^{I K}-\tilde{\alpha}_{x y}^{I} \wp_{y}^{J K}\right. \\
\left.-\tilde{\alpha}_{x y}^{K} \wp_{y}^{K K}\right](\varsigma-1) v_{4}-\sum_{y=1}^{n}\left[\tilde{\alpha}_{y x}^{R} \wp_{x}^{I R}+\tilde{\alpha}_{y x}^{I} \wp_{x}^{R R}+\tilde{\alpha}_{y x}^{I} \wp_{x}^{K R}-\tilde{\alpha}_{y x}^{K} \wp_{x}^{J R}\right] \frac{1}{v_{5}^{S-1}}-\sum_{y=1}^{n}\left[\tilde{\alpha}_{y x}^{R} \wp_{x}^{J R}\right. \\
\left.+\tilde{\alpha}_{y x}^{J} \wp_{x}^{R R}+\tilde{\alpha}_{y x}^{K} \wp_{x}^{I R}-\tilde{\alpha}_{y x}^{I} \wp_{x}^{K R}\right] \frac{1}{v_{9}^{S-1}}-\sum_{y=1}^{n}\left[\tilde{\alpha}_{y x}^{R} \wp_{x}^{K R}+\tilde{\alpha}_{y x}^{I} \wp_{x}^{J R}+\tilde{\alpha}_{y x}^{K} \wp_{x}^{R R}-\tilde{\alpha}_{y x}^{I} \wp_{x}^{I R}\right] \frac{1}{v_{13}^{\zeta-1}}>0,
\end{array}\right.
$$




$$
\begin{aligned}
& \left\{\begin{array}{l}
\mathbf{a}_{x} \zeta-\sum_{y=1}^{n}\left[\tilde{\alpha}_{y x}^{R} \wp_{x}^{R I}-\tilde{\alpha}_{y x}^{I} \wp_{x}^{I I}-\tilde{\alpha}_{y x}^{J} \wp_{x}^{J I}-\tilde{\alpha}_{y x}^{K} \wp_{x}^{K I}\right] \frac{1}{v_{2}^{\varsigma-1}}-\sum_{y=1}^{n}\left[\tilde{\alpha}_{x y}^{R} \wp_{y}^{I R}+\tilde{\alpha}_{x y}^{I} \wp_{y}^{R R}\right. \\
\left.+\tilde{\alpha}_{x y}^{J} \wp_{y}^{K R}-\tilde{\alpha}_{x y}^{K} \wp_{y}^{J R}\right](\varsigma-1) v_{5}-\sum_{y=1}^{n}\left[\tilde{\alpha}_{x y}^{R} \wp_{y}^{I I}+\tilde{\alpha}_{x y}^{I} \wp_{y}^{R I}+\tilde{\alpha}_{x y}^{J} \wp_{y}^{K I}-\tilde{\alpha}_{x y}^{K} \wp_{y}^{J I}\right](\varsigma-1) v_{6} \\
-\sum_{y=1}^{n}\left[\tilde{\alpha}_{y x}^{R} \wp_{x}^{I I}-\tilde{\alpha}_{y x}^{I} \wp_{x}^{R I}+\tilde{\alpha}_{y x}^{J} \wp_{x}^{K I}-\tilde{\alpha}_{y x}^{K} \wp_{x}^{I I}\right] \frac{1}{v_{6}^{\zeta-1}}-\sum_{y=1}^{n}\left[\tilde{\alpha}_{x y}^{R} \wp_{y}^{I J}+\tilde{\alpha}_{x y}^{I I} \wp_{y}^{R J}+\tilde{\alpha}_{x y}^{J} \wp_{y}^{K J}\right. \\
\left.-\tilde{\alpha}_{x y}^{K} \wp_{y}^{J J}\right](\varsigma-1) v_{7}-\sum_{y=1}^{n}\left[\tilde{\alpha}_{x y}^{R} \wp_{y}^{I K}+\tilde{\alpha}_{x y}^{I} \wp_{y}^{R K}+\tilde{\alpha}_{x y}^{J} \wp_{y}^{K K}-\tilde{\alpha}_{x y}^{K} \wp_{y}^{J K}\right](\varsigma-1) v_{8}-\sum_{y=1}^{n}\left[\tilde{\alpha}_{y x}^{R} \wp_{x}^{J I}\right. \\
\left.+\tilde{\alpha}_{y x}^{J} \wp_{x}^{R I}+\tilde{\alpha}_{y x}^{K} \wp_{x}^{I I}-\tilde{\alpha}_{y x}^{I} \wp_{x}^{K I}\right] \frac{1}{v_{10}^{S-1}}-\sum_{y=1}^{n}\left[\tilde{\alpha}_{y x}^{R} \wp_{x}^{K I}+\tilde{\alpha}_{y x}^{I} \wp_{x}^{J I}+\tilde{\alpha}_{y x}^{K} \wp_{x}^{R I}-\tilde{\alpha}_{y x}^{J} \wp_{x}^{I I}\right] \frac{1}{v_{14}^{S-1}}>0,
\end{array}\right. \\
& \left\{\begin{array}{l}
\mathbf{a}_{x} \varsigma-\sum_{y=1}^{n}\left[\tilde{\alpha}_{y x}^{R} \wp_{x}^{R J}-\tilde{\alpha}_{y x}^{I} \wp_{x}^{I J}-\tilde{\alpha}_{y x}^{J} \wp_{x}^{J J}-\tilde{\alpha}_{y x}^{K} \wp_{x}^{K J}\right] \frac{1}{v_{3}^{s-1}}-\sum_{y=1}^{n}\left[\tilde{\alpha}_{y x}^{R} \wp_{x}^{I J}+\tilde{\alpha}_{y x}^{I} \wp_{x}^{R J}+\tilde{\alpha}_{y x}^{J} \wp_{x}^{K J}\right. \\
\left.-\tilde{\alpha}_{y x}^{K} \wp_{x}^{J J}\right] \frac{1}{v_{7}^{S-1}}-\sum_{y=1}^{n}\left[\tilde{\alpha}_{x y}^{R} \wp_{y}^{J R}+\tilde{\alpha}_{x y}^{J} \wp_{y}^{R R}+\tilde{\alpha}_{x y}^{K} \wp_{y}^{I R}-\tilde{\alpha}_{x y}^{I} \wp_{y}^{K R}\right](\varsigma-1) v_{9}-\sum_{y=1}^{n}\left[\tilde{\alpha}_{x y}^{R} \wp_{y}^{J I}\right. \\
\left.+\tilde{\alpha}_{x y}^{J} \wp_{y}^{R I}+\tilde{\alpha}_{x y}^{K} \wp_{y}^{I I}-\tilde{\alpha}_{x y}^{I} \wp_{y}^{K I}\right](\varsigma-1) v_{10}-\sum_{y=1}^{n}\left[\tilde{\alpha}_{x y}^{R} \wp_{y}^{J J}-\tilde{\alpha}_{x y}^{J} \wp_{y}^{R J}+\tilde{\alpha}_{x y}^{K} \wp_{y}^{I J}-\tilde{\alpha}_{x y}^{I} \wp_{y}^{K J}\right](\varsigma-1) v_{11} \\
-\sum_{y=1}^{n}\left[\tilde{\alpha}_{y x}^{R} \wp_{x}^{J J}+\tilde{\alpha}_{y x}^{I} \wp_{x}^{R J}+\tilde{\alpha}_{y x}^{K} \wp_{x}^{I J}-\tilde{\alpha}_{y x}^{I} \wp_{x}^{K J}\right] \frac{1}{v_{11}^{s-1}}-\sum_{y=1}^{n}\left[\tilde{\alpha}_{x y}^{R} \wp_{y}^{J K}+\tilde{\alpha}_{x y}^{J} \wp_{y}^{R K}+\tilde{\alpha}_{x y}^{K} \wp_{y}^{I K}\right. \\
\left.-\tilde{\alpha}_{x y}^{I} \wp_{y}^{K K}\right](\varsigma-1) v_{12}-\sum_{y=1}^{n}\left[\tilde{\alpha}_{y x}^{R} \wp_{x}^{K J}+\tilde{\alpha}_{y x}^{I} \wp_{x}^{J J}+\tilde{\alpha}_{y x}^{K} \wp_{x}^{R J}-\tilde{\alpha}_{y x}^{J} \wp_{x}^{I J}\right] \frac{1}{v_{15}^{S-1}}>0,
\end{array}\right. \\
& \left\{\begin{array}{l}
\mathbf{a}_{x} \varsigma-\sum_{y=1}^{n}\left[\tilde{\alpha}_{y x}^{R} \wp_{x}^{R K}-\tilde{\alpha}_{y x}^{I} \wp_{x}^{I K}-\tilde{\alpha}_{y x}^{J} \wp_{x}^{J K}-\tilde{\alpha}_{y x}^{K} \wp_{x}^{K K}\right] \frac{1}{v_{4}^{S-1}}-\sum_{y=1}^{n}\left[\tilde{\alpha}_{y x}^{R} \wp_{x}^{I K}+\tilde{\alpha}_{y x}^{I} \wp_{x}^{R K}+\tilde{\alpha}_{y x}^{J} \wp_{x}^{K K}\right. \\
\left.-\tilde{\alpha}_{y x}^{K} \wp_{x}^{J K}\right] \frac{1}{v_{8}^{S-1}}-\sum_{y=1}^{n}\left[\tilde{\alpha}_{y x}^{R} \wp_{x}^{J K}+\tilde{\alpha}_{y x}^{J} \wp_{x}^{R K}+\tilde{\alpha}_{y x}^{K} \wp_{x}^{I K}-\tilde{\alpha}_{y x}^{I} \wp_{x}^{K K}\right] \frac{1}{v_{12}^{S-1}}-\sum_{y=1}^{n}\left[\tilde{\alpha}_{x y}^{R} \wp_{y}^{K R}+\tilde{\alpha}_{x y}^{I} \wp_{y}^{J R}\right. \\
\left.+\tilde{\alpha}_{x y}^{K} \wp_{y}^{R R}-\tilde{\alpha}_{x y}^{J} \wp_{y}^{I R}\right](\varsigma-1) v_{13}-\sum_{y=1}^{n}\left[\tilde{\alpha}_{x y}^{R} \wp_{y}^{K I}+\tilde{\alpha}_{x y}^{I} \wp_{y}^{J I}+\tilde{\alpha}_{x y}^{K} \wp_{y}^{R I}-\tilde{\alpha}_{x y}^{J} \wp_{y}^{I I}\right](\varsigma-1) v_{14} \\
-\sum_{y=1}^{n}\left[\tilde{\alpha}_{x y}^{R} \wp_{y}^{K J}+\tilde{\alpha}_{x y}^{I} \wp_{y}^{J J}+\tilde{\alpha}_{x y}^{K} \wp_{y}^{R J}-\tilde{\alpha}_{x y}^{I} \wp_{y}^{I J}\right](\varsigma-1) v_{15}-\sum_{y=1}^{n}\left[\tilde{\alpha}_{x y}^{R} \wp_{y}^{K K}+\tilde{\alpha}_{x y}^{I} \wp_{y}^{J K}+\tilde{\alpha}_{x y}^{K} \wp_{y}^{R K}\right. \\
\left.-\tilde{\alpha}_{x y}^{J} \wp_{y}^{I K}\right](\varsigma-1) v_{16}-\sum_{y=1}^{n}\left[\tilde{\alpha}_{y x}^{R} \wp_{x}^{K K}+\tilde{\alpha}_{y x}^{I} \wp_{x}^{J K}+\tilde{\alpha}_{y x}^{K} \wp_{x}^{R K}-\tilde{\alpha}_{y x}^{J} \wp_{x}^{I K}\right] \frac{1}{v_{16}^{s-1}}>0,
\end{array}\right.
\end{aligned}
$$

where $\tilde{\alpha}_{x y}^{R}=\max \left\{\left|\hat{\mathbf{b}}_{x y}^{R}\right|,\left|\grave{\mathbf{b}}_{x y}^{R}\right|\right\}, \quad \tilde{\alpha}_{x y}^{I}=\max \left\{\left|\dot{\mathbf{b}}_{x y}^{I}\right|,\left|\grave{\mathbf{b}}_{x y}^{I}\right|\right\}, \quad \tilde{\alpha}_{x y}^{I}=\max \left\{\left|\hat{\mathbf{b}}_{x y}^{I}\right|,\left|\grave{\mathbf{b}}_{x y}^{I}\right|\right\}, \quad \tilde{\alpha}_{x y}^{K}=$ $\max \left\{\left|\mathfrak{b}_{x y}^{K}\right|,\left|\grave{\mathbf{b}}_{x y}^{K}\right|\right\}$, then the NN (7) is globally Mittag-Leffler stable.

The detailed proof of Theorem 1 can be referred to in Appendix A.

\subsection{Global Mittag-Leffler Stabilization}

In this subsection, we will give sufficient criteria to stabilize the FOQVMNNs (3). Noting that, if the parameters values $\mathbf{a}_{x}, \sum_{y=1}^{n} \alpha_{x y}, x \in N$ for FOQVMNNs are appropriately chosen, the dynamics of (3) may lead to chaos. Consider the NNs as follows:

$$
{ }^{C} \mathcal{D}_{0, t}^{\sigma} w_{x}(t)=-\mathbf{a}_{x} w_{x}(t)+\sum_{y=1}^{n} \alpha_{x y} g_{y}\left(w_{y}(t)\right)+u_{x}(t), t>0, x \in N,
$$

where $u_{x}(t)$ is the external input. The state feedback stabilizing control law is defined by

$$
u_{x}(t)=\mu_{x} w_{x}(t), x \in N
$$

Take controller (14) in NN (13); then, it becomes

$$
{ }^{C} \mathcal{D}_{0, t}^{\sigma} w_{x}(t)=-\mathbf{a}_{x} w_{x}(t)+\sum_{y=1}^{n} \alpha_{x y} g_{y}\left(w_{y}(t)\right)+\mu_{x} w_{x}(t), t>0, x \in N
$$


Then, the NN (15) can be separated into real and imaginary parts:

$$
\left\{\begin{aligned}
{ }^{C} \mathcal{D}_{0, t}^{\sigma} a_{x}(t)= & -\mathbf{a}_{x} a_{x}(t)+\sum_{y=1}^{n} \alpha_{x y}^{R}\left(a_{x}(t)\right) g_{y}^{R}\left(a_{y}(t), b_{y}(t), c_{y}(t), d_{y}(t)\right)-\sum_{y=1}^{n} \alpha_{x y}^{I}\left(b_{x}(t)\right) g_{y}^{I}\left(a_{y}(t), b_{y}(t), c_{y}(t), d_{y}(t)\right) \\
& -\sum_{y=1}^{n} \alpha_{x y}^{J}\left(c_{x}(t)\right) g_{y}^{I}\left(a_{y}(t), b_{y}(t), c_{y}(t), d_{y}(t)\right)-\sum_{y=1}^{n} \alpha_{x y}^{K}\left(d_{x}(t)\right) g_{y}^{K}\left(a_{y}(t), b_{y}(t), c_{y}(t), d_{y}(t)\right)+\mu_{x} a_{x}(t) \\
{ }^{C} \mathcal{D}_{0, t}^{\sigma} b_{x}(t)= & -\mathbf{a}_{x} b_{x}(t)+\sum_{y=1}^{n} \alpha_{x y}^{R}\left(a_{x}(t)\right) g_{y}^{I}\left(a_{y}(t), b_{y}(t), c_{y}(t), d_{y}(t)\right)+\sum_{y=1}^{n} \alpha_{x y}^{I}\left(b_{x}(t)\right) g_{y}^{R}\left(a_{y}(t), b_{y}(t), c_{y}(t), d_{y}(t)\right) \\
& +\sum_{y=1}^{n} \alpha_{x y}^{J}\left(c_{x}(t)\right) g_{y}^{K}\left(a_{y}(t), b_{y}(t), c_{y}(t), d_{y}(t)\right)-\sum_{y=1}^{n} \alpha_{x y}^{K}\left(d_{x}(t)\right) g_{y}^{J}\left(a_{y}(t), b_{y}(t), c_{y}(t), d_{y}(t)\right)+\mu_{x} b_{x}(t) \\
\mathcal{C}_{\mathcal{D}_{0, t}}^{\sigma} c_{x}(t)=\quad & -\mathbf{a}_{x} c_{x}(t)+\sum_{y=1}^{n} \alpha_{x y}^{R}\left(a_{x}(t)\right) g_{y}^{I}\left(a_{y}(t), b_{y}(t), c_{y}(t), d_{y}(t)\right)+\sum_{y=1}^{n} \alpha_{x y}^{J}\left(c_{x}(t)\right) g_{y}^{R}\left(a_{y}(t), b_{y}(t), c_{y}(t), d_{y}(t)\right) \\
& +\sum_{y=1}^{n} \alpha_{x y}^{K}\left(d_{x}(t)\right) g_{y}^{I}\left(a_{y}(t), b_{y}(t), c_{y}(t), d_{y}(t)\right)-\sum_{y=1}^{n} \alpha_{x y}^{I}\left(b_{x}(t)\right) g_{y}^{K}\left(a_{y}(t), b_{y}(t), c_{y}(t), d_{y}(t)\right)+\mu_{x} c_{x}(t) \\
{ }^{C} \mathcal{D}_{0, t}^{\sigma} d_{x}(t)= & -\mathbf{a}_{x} d_{x}(t)+\sum_{y=1}^{n} \alpha_{x y}^{R}\left(a_{x}(t)\right) g_{y}^{K}\left(a_{y}(t), b_{y}(t), c_{y}(t), d_{y}(t)\right)+\sum_{y=1}^{n} \alpha_{x y}^{I}\left(b_{x}(t)\right) g_{y}^{J}\left(a_{y}(t), b_{y}(t), c_{y}(t), d_{y}(t)\right) \\
& +\sum_{y=1}^{n} \alpha_{x y}^{K}\left(d_{x}(t)\right) g_{y}^{R}\left(a_{y}(t), b_{y}(t), c_{y}(t), d_{y}(t)\right)-\sum_{y=1}^{n} \alpha_{x y}^{J}\left(c_{x}(t)\right) g_{y}^{I}\left(a_{y}(t), b_{y}(t), c_{y}(t), d_{y}(t)\right)+\mu_{x} d_{x}(t)
\end{aligned}\right.
$$

We can rewrite $\mathrm{NN}(16)$ in the vector form as follows:

$$
\left\{\begin{aligned}
{ }^{C} \mathcal{D}_{0, t}^{\sigma} a(t)= & -\mathbf{A} a(t)+\mathbf{B}^{R}(a(t)) g^{R}(a(t), b(t), c(t), d(t))-\mathbf{B}^{I}(b(t)) g^{I}(a(t), b(t), c(t), d(t)) \\
& -\mathbf{B}^{J}(c(t)) g^{J}(a(t), b(t), c(t), d(t))-\mathbf{B}^{K}(d(t)) g^{K}(a(t), b(t), c(t), d(t))+\mu a(t) \\
{ }^{C} \mathcal{D}_{0, t}^{\sigma} b(t)= & -\mathbf{A} b(t)+\mathbf{B}^{R}(a(t)) g^{I}(a(t), b(t), c(t), d(t))+\mathbf{B}^{I}(b(t)) g^{R}(a(t), b(t), c(t), d(t)) \\
& +\mathbf{B}^{J}(c(t)) g^{K}(a(t), b(t), c(t), d(t))-\mathbf{B}^{K}(d(t)) g^{J}(a(t), b(t), c(t), d(t))+\mu b(t) \\
{ }^{C} \mathcal{D}_{0, t}^{\sigma} c(t)= & -\mathbf{A} c(t)+\mathbf{B}^{R}(a(t)) g^{J}(a(t), b(t), c(t), d(t))+\mathbf{B}^{J}(c(t)) g^{R}(a(t), b(t), c(t), d(t)) \\
& +\mathbf{B}^{K}(d(t)) g^{I}(a(t), b(t), c(t), d(t))-\mathbf{B}^{I}(b(t)) g^{K}(a(t), b(t), c(t), d(t))+\mu c(t) \\
{ }^{C} \mathcal{D}_{0, t}^{\sigma} d(t)= & -\mathbf{A} d(t)+\mathbf{B}^{R}(a(t)) g^{K}(a(t), b(t), c(t), d(t))+\mathbf{B}^{I}(b(t)) g^{J}(a(t), b(t), c(t), d(t)) \\
& +\mathbf{B}^{K}(d(t)) g^{R}(a(t), b(t), c(t), d(t))-\mathbf{B}^{J}(c(t)) g^{I}(a(t), b(t), c(t), d(t))+\mu d(t)
\end{aligned}\right.
$$

where $\mu=\operatorname{diag}\left\{\mu_{1}, \ldots, \mu_{n}\right\}$.

In the following, we will derive sufficient conditions to guarantee the global Mittag-Leffler stabilization of considered FOQVNNs (16).

Theorem 2. If there exist constants $\varsigma>1$ and $v_{\ell}>0, \ell=1,2, \ldots, 16$, such that, for $x \in N$,

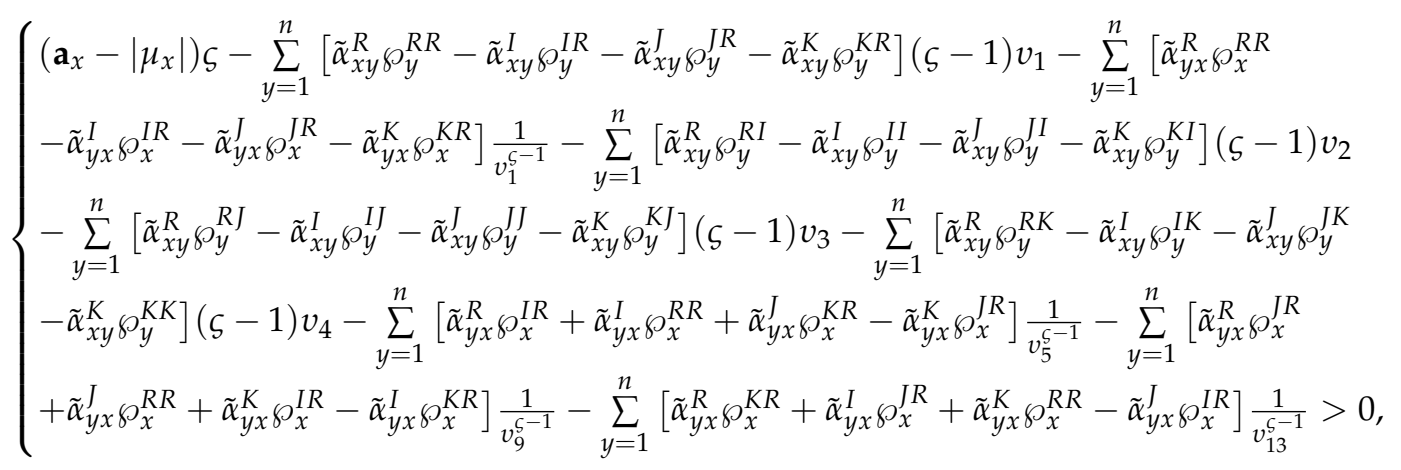




$$
\begin{aligned}
& \left\{\begin{array}{l}
\left(\mathbf{a} x-\left|\mu_{x}\right|\right) \varsigma-\sum_{y=1}^{n}\left[\tilde{\alpha}_{y x}^{R} \wp_{x}^{R I}-\tilde{\alpha}_{y x}^{I} \wp_{x}^{I I}-\tilde{\alpha}_{y x}^{I} \wp_{x}^{J I}-\tilde{\alpha}_{y x}^{K} \wp_{x}^{K I}\right] \frac{1}{v_{2}^{S-1}}-\sum_{y=1}^{n}\left[\tilde{\alpha}_{x y}^{R} \wp_{y}^{I R}+\tilde{\alpha}_{x y}^{I} \wp_{y}^{R R}\right. \\
\left.+\tilde{\alpha}_{x y}^{J} \wp_{y}^{K R}-\tilde{\alpha}_{x y}^{K} \wp_{y}^{J R}\right](\varsigma-1) v_{5}-\sum_{y=1}^{n}\left[\tilde{\alpha}_{x y}^{R} \wp_{y}^{I I}+\tilde{\alpha}_{x y}^{I} \wp_{y}^{R I}+\tilde{\alpha}_{x y}^{J} \wp_{y}^{K I}-\tilde{\alpha}_{x y}^{K} \wp_{y}^{J I}\right](\varsigma-1) v_{6} \\
-\sum_{y=1}^{n}\left[\tilde{\alpha}_{y x}^{R} \wp_{x}^{I I}-\tilde{\alpha}_{y x}^{I} \wp_{x}^{R I}+\tilde{\alpha}_{y x}^{I} \wp_{x}^{K I}-\tilde{\alpha}_{y x}^{K} \wp_{x}^{J I}\right] \frac{1}{v_{6}^{s-1}}-\sum_{y=1}^{n}\left[\tilde{\alpha}_{x y}^{R} \wp_{y}^{I J}+\tilde{\alpha}_{x y}^{I} \wp_{y}^{R J}+\tilde{\alpha}_{x y}^{J} \wp_{y}^{K J}\right. \\
\left.-\tilde{\alpha}_{x y}^{K} \wp_{y}^{J J}\right](\varsigma-1) v_{7}-\sum_{y=1}^{n}\left[\tilde{\alpha}_{x y}^{R} \wp_{y}^{I K}+\tilde{\alpha}_{x y}^{I} \wp_{y}^{R K}+\tilde{\alpha}_{x y}^{J} \wp_{y}^{K K}-\tilde{\alpha}_{x y}^{K} \wp_{y}^{J K}\right](\varsigma-1) v_{8}-\sum_{y=1}^{n}\left[\tilde{\alpha}_{y x}^{R} \wp_{x}^{J I}\right. \\
\left.+\tilde{\alpha}_{y x}^{J} \wp_{x}^{R I}+\tilde{\alpha}_{y x}^{K} \wp_{x}^{I I}-\tilde{\alpha}_{y x}^{I} \wp_{x}^{K I}\right] \frac{1}{v_{10}^{\varsigma-1}}-\sum_{y=1}^{n}\left[\tilde{\alpha}_{y x}^{R} \wp_{x}^{K I}+\tilde{\alpha}_{y x}^{I} \wp_{x}^{J I}+\tilde{\alpha}_{y x}^{K} \wp_{x}^{R I}-\tilde{\alpha}_{y x}^{J} \wp_{x}^{I I}\right] \frac{1}{v_{14}^{S-1}}>0,
\end{array}\right. \\
& \left\{\begin{array}{l}
\left(\mathbf{a}_{x}-\left|\mu_{x}\right|\right) \varsigma-\sum_{y=1}^{n}\left[\tilde{\alpha}_{y x}^{R} \wp_{x}^{R J}-\tilde{\alpha}_{y x}^{I} \wp_{x}^{I J}-\tilde{\alpha}_{y x}^{J} \wp_{x}^{J J}-\tilde{\alpha}_{y x}^{K} \wp_{x}^{K J}\right] \frac{1}{v_{3}^{S-1}}-\sum_{y=1}^{n}\left[\tilde{\alpha}_{y x}^{R} \wp_{x}^{I J}+\tilde{\alpha}_{y x}^{I} \wp_{x}^{R J}\right. \\
\left.+\tilde{\alpha}_{y x}^{J} \wp_{x}^{K J}-\tilde{\alpha}_{y x}^{K} \wp_{x}^{J J}\right] \frac{1}{v_{7}^{S-1}}-\sum_{y=1}^{n}\left[\tilde{\alpha}_{x y}^{R} \wp_{y}^{J R}+\tilde{\alpha}_{x y}^{J} \wp_{y}^{R R}+\tilde{\alpha}_{x y}^{K} \wp_{y}^{I R}-\tilde{\alpha}_{x y}^{I} \wp_{y}^{K R}\right](\varsigma-1) v_{9}-\sum_{y=1}^{n}\left[\tilde{\alpha}_{x y}^{R} \wp_{y}^{J I}\right. \\
\left.+\tilde{\alpha}_{x y}^{J} \wp_{y}^{R I}+\tilde{\alpha}_{x y}^{K} \wp_{y}^{I I}-\tilde{\alpha}_{x y}^{I} \wp_{y}^{K I}\right](\varsigma-1) v_{10}-\sum_{y=1}^{n}\left[\tilde{\alpha}_{x y}^{R} \wp_{y}^{J J}-\tilde{\alpha}_{x y}^{J} \wp_{y}^{R J}+\tilde{\alpha}_{x y}^{K} \wp_{y}^{I J}-\tilde{\alpha}_{x y}^{I} \wp_{y}^{K J}\right](\varsigma-1) v_{11} \\
-\sum_{y=1}^{n}\left[\tilde{\alpha}_{y x}^{R} \wp_{x}^{J J}+\tilde{\alpha}_{y x}^{I} \wp_{x}^{R J}+\tilde{\alpha}_{y x}^{K} \wp_{x}^{I J}-\tilde{\alpha}_{y x}^{I} \wp_{x}^{K J}\right] \frac{1}{v_{11}^{S-1}}-\sum_{y=1}^{n}\left[\tilde{\alpha}_{x y}^{R} \wp_{y}^{J K}+\tilde{\alpha}_{x y}^{J} \wp_{y}^{R K}+\tilde{\alpha}_{x y}^{K} \wp_{y}^{I K}\right. \\
\left.-\tilde{\alpha}_{x y}^{I} \wp_{y}^{K K}\right](\varsigma-1) v_{12}-\sum_{y=1}^{n}\left[\tilde{\alpha}_{y x}^{R} \wp_{x}^{K J}+\tilde{\alpha}_{y x}^{I} \wp_{x}^{J J}+\tilde{\alpha}_{y x}^{K} \wp_{x}^{R J}-\tilde{\alpha}_{y x}^{J} \wp_{x}^{I J}\right] \frac{1}{v_{15}^{S-1}}>0,
\end{array}\right. \\
& \left\{\begin{array}{l}
\left(\mathbf{a}_{x}-\left|\mu_{x}\right|\right) \varsigma-\sum_{y=1}^{n}\left[\tilde{\alpha}_{y x}^{R} \wp_{x}^{R K}-\tilde{\alpha}_{y x}^{I} \wp_{x}^{I K}-\tilde{\alpha}_{y x}^{J} \wp_{x}^{J K}-\tilde{\alpha}_{y x}^{K} \wp_{x}^{K K}\right] \frac{1}{v_{4}^{S-1}}-\sum_{y=1}^{n}\left[\tilde{\alpha}_{y x}^{R} \wp_{x}^{I K}+\tilde{\alpha}_{y x}^{I} \wp_{x}^{R K}\right. \\
\left.+\tilde{\alpha}_{y x}^{J} \wp_{x}^{K K}-\tilde{\alpha}_{y x}^{K} \wp_{x}^{J K}\right] \frac{1}{v_{8}^{S-1}}-\sum_{y=1}^{n}\left[\tilde{\alpha}_{y x}^{R} \wp_{x}^{J K}+\tilde{\alpha}_{y x}^{J} \wp_{x}^{R K}+\tilde{\alpha}_{y x}^{K} \wp_{x}^{I K}-\tilde{\alpha}_{y x}^{I} \wp_{x}^{K K}\right] \frac{1}{v_{12}^{S-1}}-\sum_{y=1}^{n}\left[\tilde{\alpha}_{x y}^{R} \wp_{y}^{K R}\right. \\
\left.+\tilde{\alpha}_{x y}^{I} \wp_{y}^{J R}+\tilde{\alpha}_{x y}^{K} \wp_{y}^{R R}-\tilde{\alpha}_{x y}^{J} \wp_{y}^{I R}\right](\varsigma-1) v_{13} s-\sum_{y=1}^{n}\left[\tilde{\alpha}_{x y}^{R} \wp_{y}^{K I}+\tilde{\alpha}_{x y}^{I} \wp_{y}^{J I}+\tilde{\alpha}_{x y}^{K} \wp_{y}^{R I}-\tilde{\alpha}_{x y}^{J} \wp_{y}^{I I}\right](\varsigma-1) v_{14} \\
-\sum_{y=1}^{n}\left[\tilde{\alpha}_{x y}^{R} \wp_{y}^{K J}+\tilde{\alpha}_{x y}^{I} \wp_{y}^{J J}+\tilde{\alpha}_{x y}^{K} \wp_{y}^{R J}-\tilde{\alpha}_{x y}^{J} \wp_{y}^{I J}\right](\varsigma-1) v_{15}-\sum_{y=1}^{n}\left[\tilde{\alpha}_{x y}^{R} \wp_{y}^{K K}+\tilde{\alpha}_{x y}^{I} \wp_{y}^{J K}+\tilde{\alpha}_{x y}^{K} \wp_{y}^{R K}\right. \\
\left.-\tilde{\alpha}_{x y}^{J} \wp_{y}^{I K}\right](\varsigma-1) v_{16}-\sum_{y=1}^{n}\left[\tilde{\alpha}_{y x}^{R} \wp_{x}^{K K}+\tilde{\alpha}_{y x}^{I} \wp_{x}^{J K}+\tilde{\alpha}_{y x}^{K} \wp_{x}^{R K}-\tilde{\alpha}_{y x}^{J} \wp_{x}^{I K}\right] \frac{1}{v_{16}^{S-1}}>0,
\end{array}\right.
\end{aligned}
$$

where $\tilde{\alpha}_{x y}^{R}=\max \left\{\left|\hat{\mathbf{b}}_{x y}^{R}\right|,\left|\grave{\mathbf{b}}_{x y}^{R}\right|\right\}, \quad \tilde{\alpha}_{x y}^{I}=\max \left\{\left|\hat{\mathbf{b}}_{x y}^{I}\right|,\left|\grave{\mathbf{b}}_{x y}^{I}\right|\right\}, \quad \tilde{\alpha}_{x y}^{J}=\max \left\{\left|\hat{\mathbf{b}}_{x y}^{J}\right|,\left|\grave{\mathbf{b}}_{x y}^{J}\right|\right\}, \quad \tilde{\alpha}_{x y}^{K}=$ $\max \left\{\left|\hat{\mathbf{b}}_{x y}^{K}\right|,\left|\grave{\mathbf{b}}_{x y}^{K}\right|\right\}$, then system (16) is globally Mittag-Leffler stable. That is, under the condition of control law (14), system (16) can realize global Mittag-Leffler stabilization.

Proof. Take the same Lyapunov function defined in Theorem 1, and by the similar procedure presented in the proof of Theorem 1 , the conditions (18)-(21) can be easily derived by replacing $\mathbf{a}_{x}$ by $\left(\mathbf{a}_{x}-\right.$ $\left.\left|\mu_{x}\right|\right), x \in N$. Thus, it is omitted.

Remark 4. Theorem 1 reveals that the global Mittag-Leffler stability of NN (7) and Theorem 2 reveals that the global Mittag-Leffler stabilization of NN (16) with the free-weighting parameters $v_{p}(p=1, \ldots, 12)$. It is easy to know that the use of free-weighting parameters $v_{p}(p=1, \ldots 12)$ can reduce the conservativeness of theoretical results. Thus, compared with that in $[37,38,40]$, our approach is more effective and feasible.

Remark 5. In the existing works of literature, very few results have been published about FOQVMNNs. Unlike the research methods in [32,33], the QVNN model (3) is a fractional-order QVNN rather than the integer-order QVNN model in [32,33]. However, we know that the analysis methods for integer-order NNs cannot be directly used for fractional-order NNs, and, consequently, it raises many difficult problems for the design and analysis of system (3). From this point, this paper offers a better approach for studying the stability of FOQVMNNs.

\section{Illustrative Examples}

In this section, two numerical examples are given to validate the effectiveness of the obtained conditions. 
Example 1. Consider the following two-neurons FOQVMNNs:

$$
\left\{\begin{aligned}
{ }^{C} \mathcal{D}_{0, t}^{\sigma} w_{1}(t)= & -\mathbf{a}_{1} w_{1}(t)+\left[\mathbf{b}_{11}^{R}\left(w_{1}(t)\right)+\mathbf{b}_{11}^{I}\left(w_{1}(t)\right)+\mathbf{b}_{11}^{J}\left(w_{1}(t)\right)+\mathbf{b}_{11}^{K}\left(w_{1}(t)\right)\right] g_{1}\left(w_{1}(t)\right) \\
& +\left[\mathbf{b}_{12}^{R}\left(w_{1}(t)\right)+\mathbf{b}_{12}^{I}\left(w_{1}(t)\right)+\mathbf{b}_{12}^{J}\left(w_{1}(t)\right)+\mathbf{b}_{12}^{K}\left(w_{1}(t)\right)\right] g_{2}\left(w_{2}(t)\right) \\
{ }^{C} \mathcal{D}_{0, t}^{\sigma} w_{2}(t)= & -\mathbf{a}_{2} w_{2}(t)+\left[\mathbf{b}_{21}^{R}\left(w_{2}(t)\right)+\mathbf{b}_{21}^{I}\left(w_{2}(t)\right)+\mathbf{b}_{21}^{J}\left(w_{2}(t)\right)+\mathbf{b}_{21}^{K}\left(w_{2}(t)\right)\right] g_{1}\left(w_{1}(t)\right) \\
& +\left[\mathbf{b}_{22}^{R}\left(w_{2}(t)\right)+\mathbf{b}_{22}^{I}\left(w_{2}(t)\right)+\mathbf{b}_{22}^{J}\left(w_{2}(t)\right)+\mathbf{b}_{22}^{K}\left(w_{2}(t)\right)\right] g_{2}\left(w_{2}(t)\right)
\end{aligned}\right.
$$

where $\sigma=0.98, g_{x}\left(w_{x}(t)\right)=0.5 \tanh (w(t)), \mathbf{a}_{1}=1.8, \mathbf{a}_{2}=2.2$, and

$$
\begin{aligned}
& \mathbf{b}_{11}^{R}\left(w_{1}(t)\right)=\left\{\begin{array}{l}
0.2,\left|w_{1}(t)\right|<1 \\
-0.2,\left|w_{1}(t)\right|>1,
\end{array} \quad \mathbf{b}_{11}^{I}\left(w_{1}(t)\right)=\left\{\begin{array}{l}
0.5, \quad\left|w_{1}(t)\right|<1 \\
-0.5, \quad\left|w_{1}(t)\right|>1,
\end{array}\right.\right. \\
& \mathbf{b}_{11}^{J}\left(w_{1}(t)\right)=\left\{\begin{array}{l}
0.7,\left|w_{1}(t)\right|<1 \\
-0.7,\left|w_{1}(t)\right|>1,
\end{array} \quad \mathbf{b}_{11}^{K}\left(w_{1}(t)\right)=\left\{\begin{array}{l}
0.9, \quad\left|w_{1}(t)\right|<1 \\
-0.9,\left|w_{1}(t)\right|>1,
\end{array}\right.\right. \\
& \mathbf{b}_{12}^{R}\left(w_{1}(t)\right)=\left\{\begin{array}{l}
0.4,\left|w_{1}(t)\right|<1 \\
-0.4,\left|w_{1}(t)\right|>1,
\end{array} \quad \mathbf{b}_{12}^{I}\left(w_{1}(t)\right)=\left\{\begin{array}{l}
0.5, \quad\left|w_{1}(t)\right|<1 \\
-0.5,\left|w_{1}(t)\right|>1,
\end{array}\right.\right. \\
& \mathbf{b}_{12}^{J}\left(w_{1}(t)\right)=\left\{\begin{array}{l}
0.6, \quad\left|w_{1}(t)\right|<1 \\
-0.6, \quad\left|w_{1}(t)\right|>1,
\end{array} \quad \mathbf{b}_{12}^{K}\left(w_{1}(t)\right)=\left\{\begin{array}{l}
0.7,\left|w_{1}(t)\right|<1 \\
-0.7,\left|w_{1}(t)\right|>1,
\end{array}\right.\right. \\
& \mathbf{b}_{21}^{R}\left(w_{2}(t)\right)=\left\{\begin{array}{l}
0.3,\left|w_{2}(t)\right|<1 \\
-0.3,\left|w_{2}(t)\right|>1,
\end{array} \quad \mathbf{b}_{21}^{I}\left(w_{2}(t)\right)=\left\{\begin{array}{l}
0.2, \quad\left|w_{2}(t)\right|<1 \\
-0.2, \quad\left|w_{2}(t)\right|>1,
\end{array}\right.\right. \\
& \mathbf{b}_{21}^{J}\left(w_{2}(t)\right)=\left\{\begin{array}{l}
0.4,\left|w_{2}(t)\right|<1 \\
-0.4,\left|w_{2}(t)\right|>1,
\end{array} \quad \mathbf{b}_{21}^{K}\left(w_{2}(t)\right)=\left\{\begin{array}{l}
0.5, \quad\left|w_{2}(t)\right|<1 \\
-0.5, \quad\left|w_{2}(t)\right|>1,
\end{array}\right.\right. \\
& \mathbf{b}_{22}^{R}\left(w_{2}(t)\right)=\left\{\begin{array}{l}
0.14,\left|w_{2}(t)\right|<1 \\
-0.14,\left|w_{2}(t)\right|>1,
\end{array} \quad \mathbf{b}_{22}^{I}\left(w_{2}(t)\right)=\left\{\begin{array}{l}
0.33,\left|w_{2}(t)\right|<1 \\
-0.33,\left|w_{2}(t)\right|>1,
\end{array}\right.\right. \\
& \mathbf{b}_{22}^{J}\left(w_{2}(t)\right)=\left\{\begin{array}{l}
0.20,\left|w_{2}(t)\right|<1 \\
-0.20,\left|w_{2}(t)\right|>1,
\end{array} \quad \mathbf{b}_{22}^{K}\left(w_{2}(t)\right)=\left\{\begin{array}{l}
0.6,\left|w_{2}(t)\right|<1 \\
-0.6, \quad\left|w_{2}(t)\right|>1 .
\end{array}\right.\right.
\end{aligned}
$$

In addition, choose $\wp_{y}^{R R}=\wp_{y}^{I I}=\wp_{y}^{J J}=\wp_{y}^{K K}=1, \wp_{y}^{R I}=\wp_{y}^{R J}=\wp_{y}^{R K}=0, \wp_{y}^{I R}=\wp_{y}^{I J}=\wp_{y}^{I K}=0$, $\wp_{y}^{J R}=\wp_{y}^{J I}=\wp_{y}^{J K}=0, \wp_{y}^{K R}=\wp_{y}^{K I}=\wp_{y}^{K J}=0, y=1,2$. and $\varsigma=2, v_{\ell}=1, \ell=1,2, \ldots, 16$. According to Theorem 1, the NN (22) can realize global Mittag-Leffler stable. The initial values are taken as $w_{1}(0)=0.3+0.4 i+0.5 j-0.6 k$ and $w_{2}(0)=-0.1-0.3 i-0.2 j+0.3 k$. Figures $1-4$ show the state trajectory of NN (22). As plotted in Figures 1-4, state trajectory of NN (22) converges to the equilibrium point. 


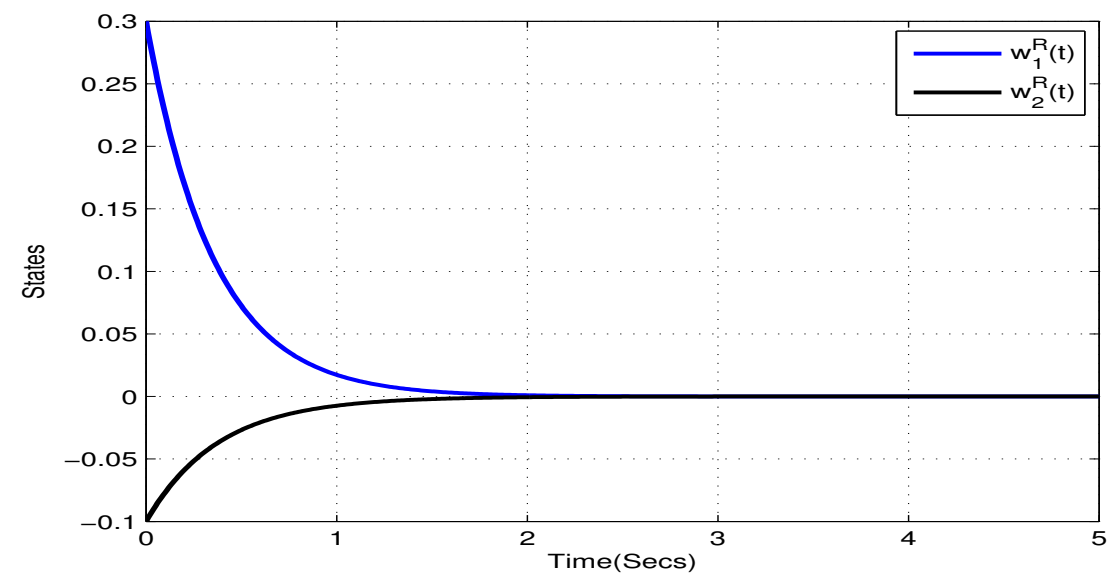

Figure 1. State trajectory of $w_{1}^{R}(t), w_{2}^{R}(t)$ of systems (22) in Example 1.

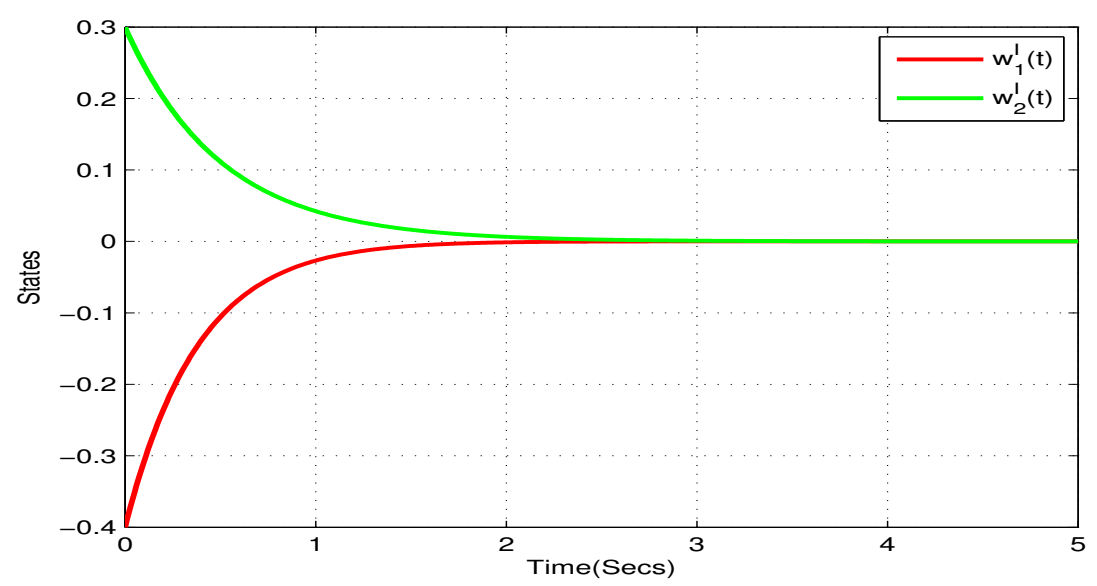

Figure 2. State trajectory of $w_{1}^{I}(t), w_{2}^{I}(t)$ of systems (22) in Example 1.

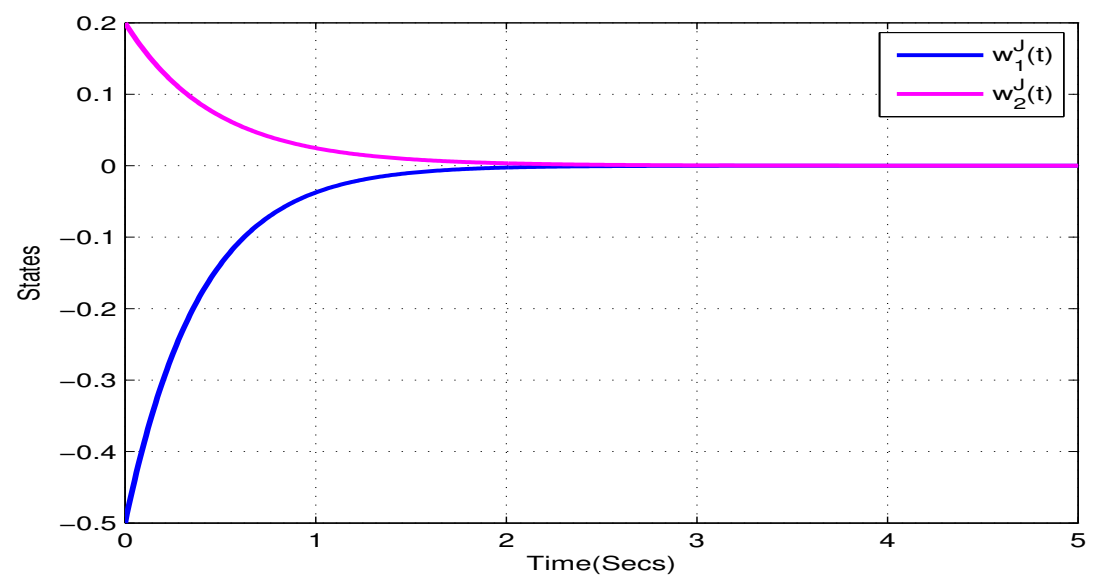

Figure 3. State trajectory of $w_{1}^{K}(t), w_{2}^{K}(t)$ of systems (22) in Example 1. 


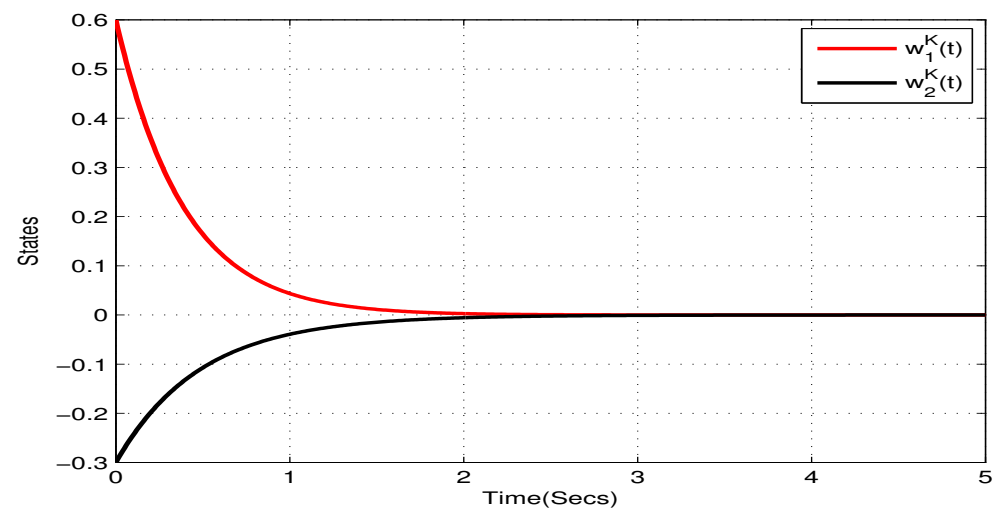

Figure 4. State trajectory of $w_{1}^{K}(t), w_{2}^{K}(t)$ of systems (22) in Example 1.

Example 2. Consider the following two-neurons FOQVMNNs:

$$
\left\{\begin{aligned}
{ }^{C} \mathcal{D}_{0, t}^{\sigma} w_{1}(t)= & -\mathbf{a}_{1} w_{1}(t)+\left[\mathbf{b}_{11}^{R}\left(w_{1}(t)\right)+\mathbf{b}_{11}^{I}\left(w_{1}(t)\right)+\mathbf{b}_{11}^{J}\left(w_{1}(t)\right)+\mathbf{b}_{11}^{K}\left(w_{1}(t)\right)\right] g_{1}\left(w_{1}(t)\right) \\
& +\left[\mathbf{b}_{12}^{R}\left(w_{1}(t)\right)+\mathbf{b}_{12}^{I}\left(w_{1}(t)\right)+\mathbf{b}_{12}^{J}\left(w_{1}(t)\right)+\mathbf{b}_{12}^{K}\left(w_{1}(t)\right)\right] g_{2}\left(w_{2}(t)\right)+u_{1}(t) \\
{ }^{C} \mathcal{D}_{0, t}^{\sigma} w_{2}(t)= & -\mathbf{a}_{2} w_{2}(t)+\left[\mathbf{b}_{21}^{R}\left(w_{2}(t)\right)+\mathbf{b}_{21}^{I}\left(w_{2}(t)\right)+\mathbf{b}_{21}^{J}\left(w_{2}(t)\right)+\mathbf{b}_{21}^{K}\left(w_{2}(t)\right)\right] g_{1}\left(w_{1}(t)\right) \\
& +\left[\mathbf{b}_{22}^{R}\left(w_{2}(t)\right)+\mathbf{b}_{22}^{I}\left(w_{2}(t)\right)+\mathbf{b}_{22}^{J}\left(w_{2}(t)\right)+\mathbf{b}_{22}^{K}\left(w_{2}(t)\right)\right] g_{2}\left(w_{2}(t)\right)+u_{2}(t),
\end{aligned}\right.
$$

where $\sigma=0.98, g_{x}\left(w_{x}(t)\right)=0.5 \tanh (w(t)), \mathbf{a}_{1}=0.8, \mathbf{a}_{2}=1.2$, and

$$
\begin{aligned}
& \mathbf{b}_{11}^{R}\left(w_{1}(t)\right)=\left\{\begin{array}{l}
0.4,\left|w_{1}(t)\right|<1 \\
-0.4,\left|w_{1}(t)\right|>1,
\end{array} \quad \mathbf{b}_{11}^{I}\left(w_{1}(t)\right)=\left\{\begin{array}{l}
0.4,\left|w_{1}(t)\right|<1 \\
-0.4,\left|w_{1}(t)\right|>1,
\end{array}\right.\right. \\
& \mathbf{b}_{11}^{J}\left(w_{1}(t)\right)=\left\{\begin{array}{l}
0.18,\left|w_{1}(t)\right|<1 \\
-0.18,\left|w_{1}(t)\right|>1,
\end{array} \quad \mathbf{b}_{11}^{K}\left(w_{1}(t)\right)=\left\{\begin{array}{l}
0.6,\left|w_{1}(t)\right|<1 \\
-0.6,\left|w_{1}(t)\right|>1,
\end{array}\right.\right. \\
& \mathbf{b}_{12}^{R}\left(w_{1}(t)\right)=\left\{\begin{array}{l}
0.2,\left|w_{1}(t)\right|<1 \\
-0.2,\left|w_{1}(t)\right|>1,
\end{array} \quad \mathbf{b}_{12}^{I}\left(w_{1}(t)\right)=\left\{\begin{array}{l}
0.5, \quad\left|w_{1}(t)\right|<1 \\
-0.5, \quad\left|w_{1}(t)\right|>1,
\end{array}\right.\right. \\
& \mathbf{b}_{12}^{J}\left(w_{1}(t)\right)=\left\{\begin{array}{l}
0.6,\left|w_{1}(t)\right|<1 \\
-0.6,\left|w_{1}(t)\right|>1,
\end{array} \quad \mathbf{b}_{12}^{K}\left(w_{1}(t)\right)=\left\{\begin{array}{l}
0.7,\left|w_{1}(t)\right|<1 \\
-0.7,\left|w_{1}(t)\right|>1,
\end{array}\right.\right. \\
& \mathbf{b}_{21}^{R}\left(w_{2}(t)\right)=\left\{\begin{array}{l}
0.2,\left|w_{2}(t)\right|<1 \\
-0.2,\left|w_{2}(t)\right|>1,
\end{array} \quad \mathbf{b}_{21}^{I}\left(w_{2}(t)\right)=\left\{\begin{array}{l}
0.12,\left|w_{2}(t)\right|<1 \\
-0.12,\left|w_{2}(t)\right|>1,
\end{array}\right.\right. \\
& \mathbf{b}_{21}^{J}\left(w_{2}(t)\right)=\left\{\begin{array}{l}
0.24,\left|w_{2}(t)\right|<1 \\
-0.24,\left|w_{2}(t)\right|>1,
\end{array} \quad \mathbf{b}_{21}^{K}\left(w_{2}(t)\right)=\left\{\begin{array}{l}
0.35,\left|w_{2}(t)\right|<1 \\
-0.35,\left|w_{2}(t)\right|>1,
\end{array}\right.\right. \\
& \mathbf{b}_{22}^{R}\left(w_{2}(t)\right)=\left\{\begin{array}{l}
0.4,\left|w_{2}(t)\right|<1 \\
-0.4,\left|w_{2}(t)\right|>1,
\end{array} \quad \mathbf{b}_{22}^{I}\left(w_{2}(t)\right)=\left\{\begin{array}{l}
0.24,\left|w_{2}(t)\right|<1 \\
-0.24,\left|w_{2}(t)\right|>1,
\end{array}\right.\right. \\
& \mathbf{b}_{22}^{J}\left(w_{2}(t)\right)=\left\{\begin{array}{l}
0.10,\left|w_{2}(t)\right|<1 \\
-0.10,\left|w_{2}(t)\right|>1,
\end{array} \quad \mathbf{b}_{22}^{K}\left(w_{2}(t)\right)=\left\{\begin{array}{l}
0.20,\left|w_{2}(t)\right|<1 \\
-0.20,\left|w_{2}(t)\right|>1 .
\end{array}\right.\right.
\end{aligned}
$$

Take $\wp_{y}^{R R}=\wp_{y}^{I I}=\wp_{y}^{J I}=\wp_{y}^{K K}=1, \wp_{y}^{R I}=\wp_{y}^{R J}=\wp_{y}^{R K}=0, \wp_{y}^{I R}=\wp_{y}^{I J}=\wp_{y}^{I K}=0, \wp_{y}^{J R}=\wp_{y}^{J I}=$ $\wp_{y}^{J K}=0, \wp_{y}^{K R}=\wp_{y}^{K I}=\wp_{y}^{K J}=0, y=1,2$. and $\varsigma=2, v_{\ell}=1, \ell=1,2, \ldots, 16$. Figures $5-8$ show the state trajectory of $N N$ (23) without control input, respectively. As plotted in Figures 5-8, state trajectory of $N N(23)$ is divergent under the initial values $w_{1}(0)=0.6-0.9 i+0.4 j-0.5 k$ and $w_{2}(0)=0.8+1.2 i-0.7 j-0.8 k$. On the basis of above values, we can conclude $\left|\mu_{1}\right|<0.14$ and $\left|\mu_{2}\right|<0.44$. According to Theorem 2 , the NN 
(23) can realize global Mittag-Leffler stabilization when we choose the state feedback control law $u_{1}(t)=$ $0.14 w_{1}(t), u_{2}(t)=0.44 w_{2}(t)$. Figure 9 depicts the state trajectory of real parts of $N N(23)$ with state feedback control law $u_{1}(t)=0.12 a_{1}(t), u_{2}(t)=0.42 a_{2}(t)$. Similarly, Figures 10-12 shows the state trajectory of imaginary parts of NN (23) with state feedback control law, respectively $u_{1}(t)=0.12 b_{1}(t), u_{2}(t)=0.42 b_{2}(t)$, $u_{1}(t)=0.12 c_{1}(t), u_{2}(t)=0.42 c_{2}(t), u_{1}(t)=0.12 d_{1}(t), u_{2}(t)=0.42 d_{2}(t)$.

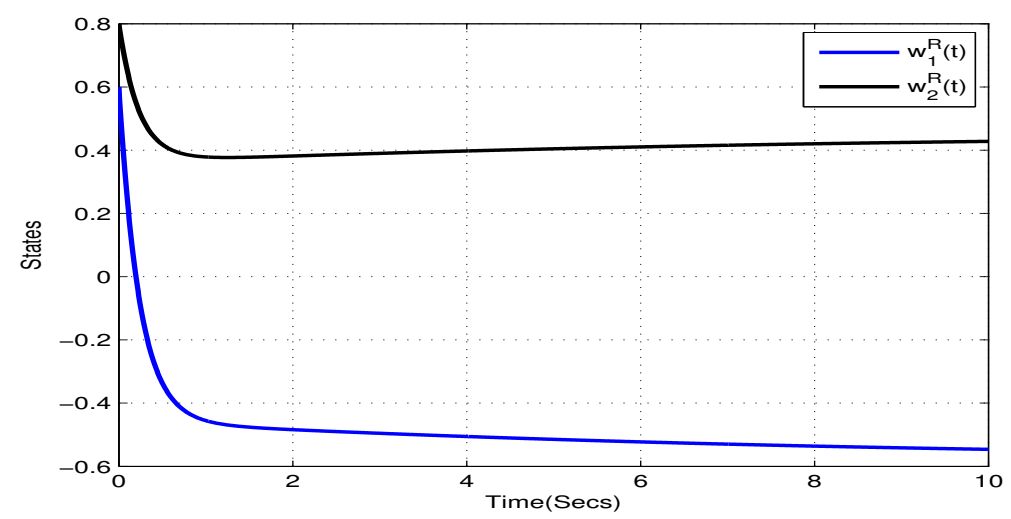

Figure 5. State trajectory of $w_{1}^{R}(t), w_{2}^{R}(t)$ of systems (23) with state feedback control $u_{1}=0, u_{2}=0$ in Example 2.

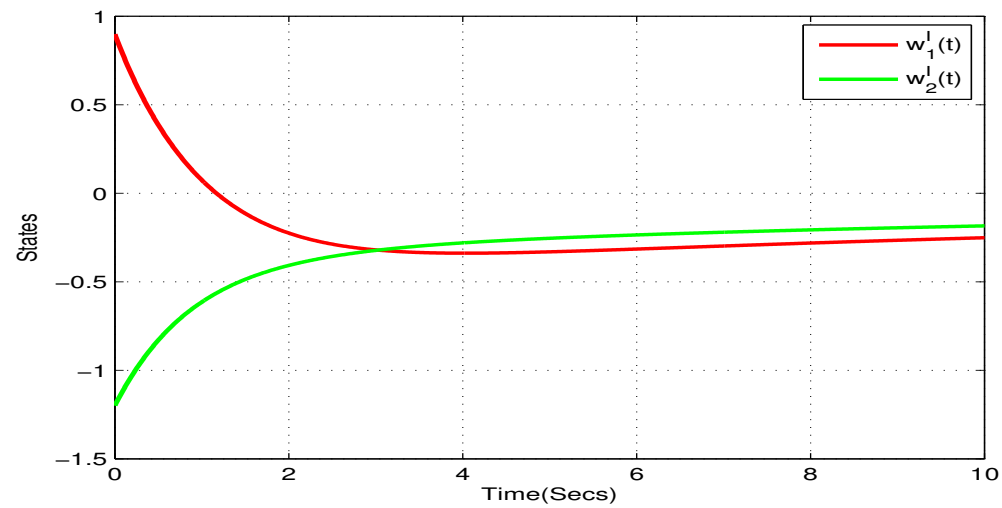

Figure 6. State trajectory of $w_{1}^{I}(t), w_{2}^{I}(t)$ of systems (23) with state feedback control $u_{1}=0, u_{2}=0$ in Example 2.

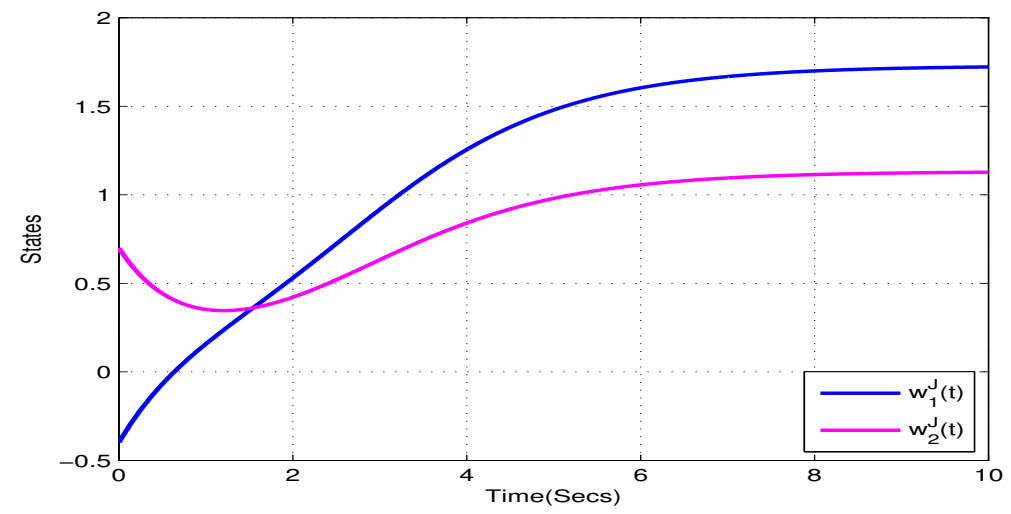

Figure 7. State trajectory of $w_{1}^{J}(t), w_{2}^{J}(t)$ of systems (23) with state feedback control $u_{1}=0, u_{2}=0$ in Example 2. 


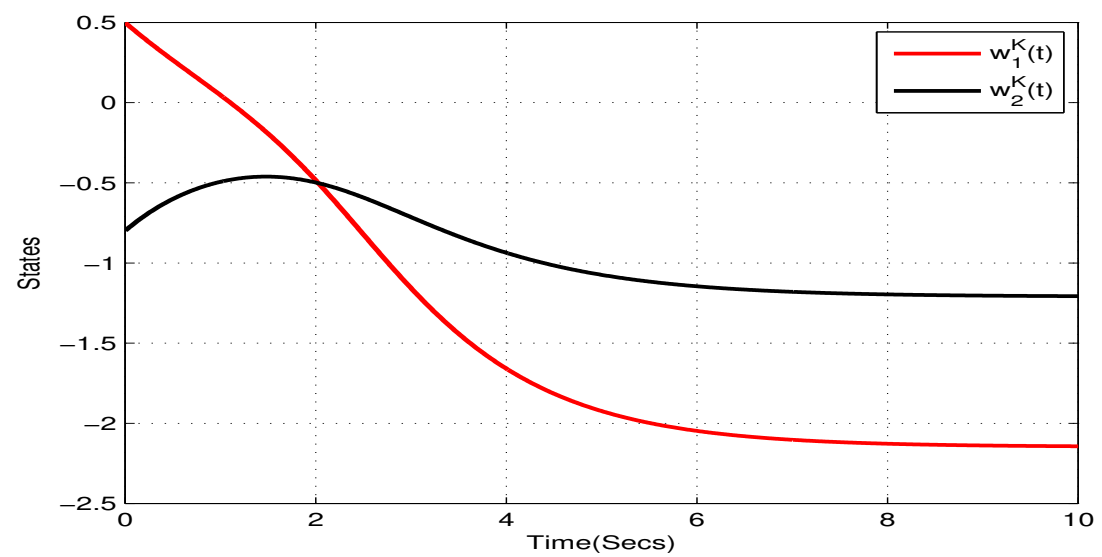

Figure 8. State trajectory of $w_{1}^{K}(t), w_{2}^{K}(t)$ of systems (23) with state feedback control $u_{1}=0, u_{2}=0$ in Example 2.

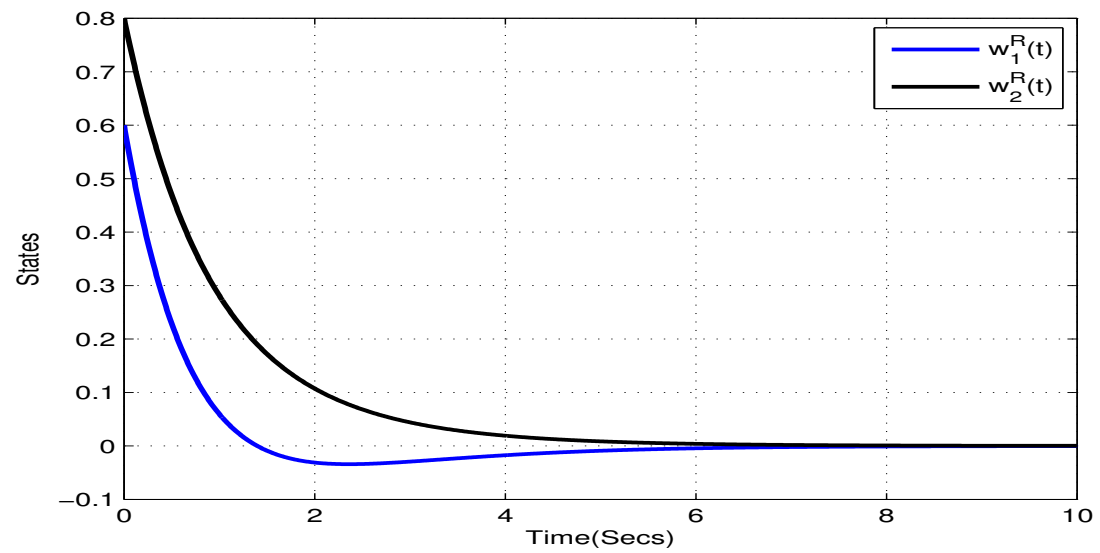

Figure 9. State trajectory of $w_{1}^{R}(t), w_{2}^{R}(t)$ of systems (23) with state feedback control $u_{1}=$ $0.12 a_{1}(t), u_{2}=0.42 a_{2}(t)$ in Example 2 .

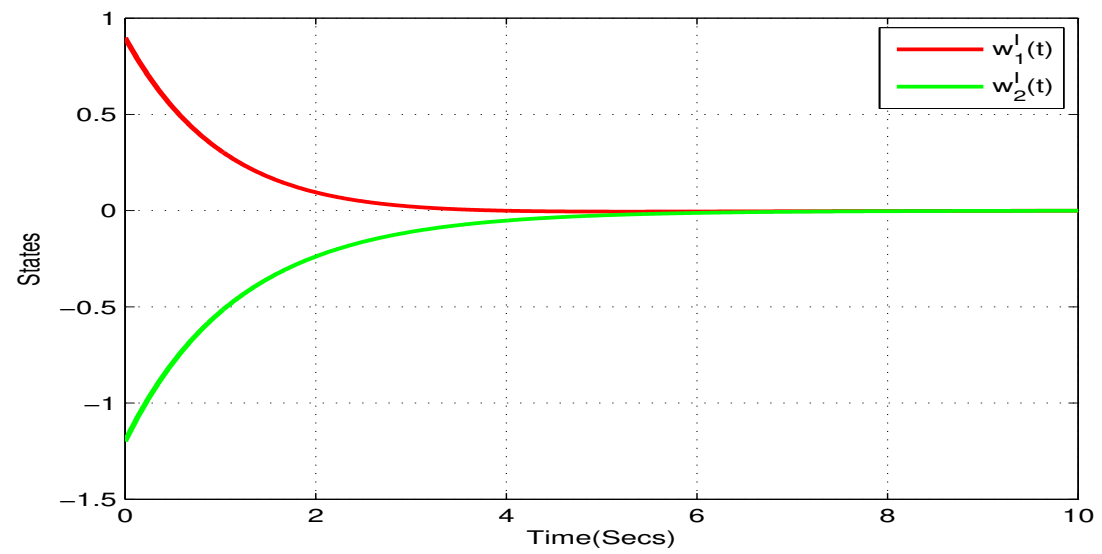

Figure 10. State trajectory of $w_{1}^{I}(t), w_{2}^{I}(t)$ of systems (23) with state feedback control $u_{1}=$ $0.12 b_{1}(t), u_{2}=0.42 b_{2}(t)$ in Example 2 . 


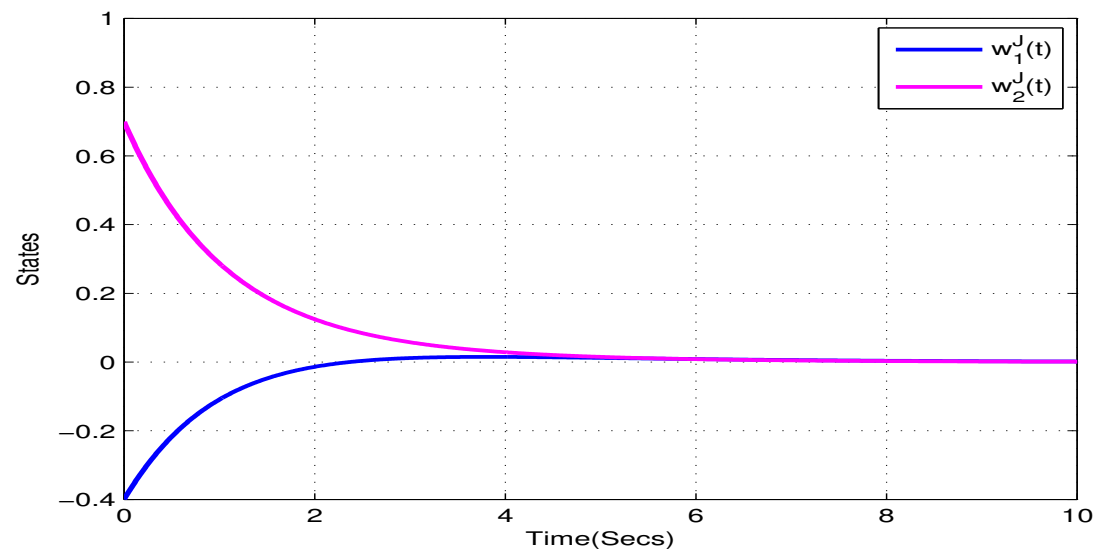

Figure 11. State trajectory of $w_{1}^{J}(t), w_{2}^{J}(t)$ of systems (23) with state feedback control $u_{1}=$ $0.12 c_{1}(t), u_{2}=0.42 c_{2}(t)$ in Example 2 .

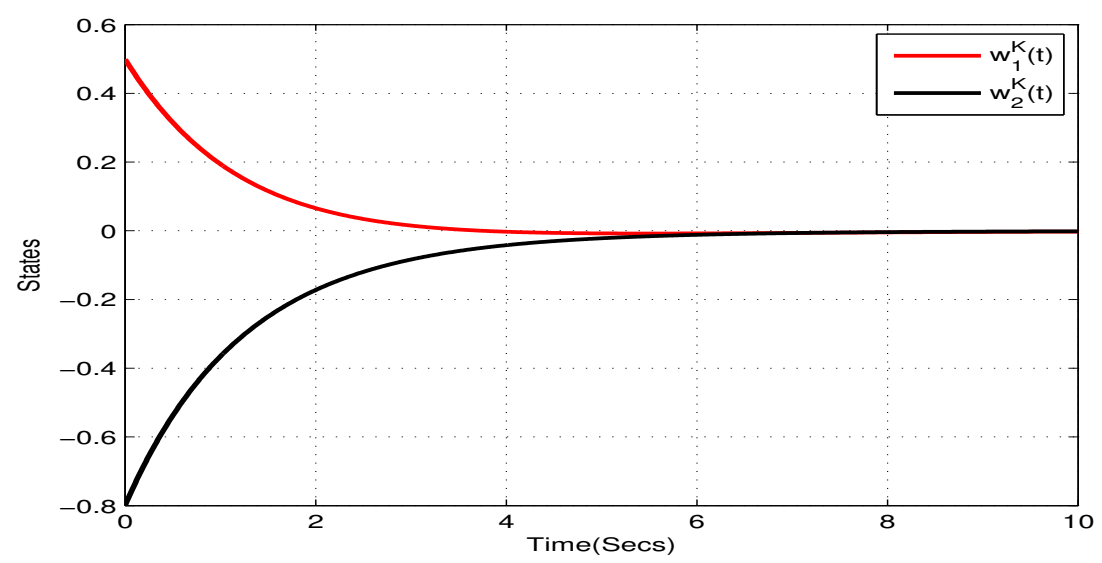

Figure 12. State trajectory of $w_{1}^{K}(t), w_{2}^{K}(t)$ of systems (23) with state feedback control $u_{1}=$ $0.12 d_{1}(t), u_{2}=0.42 d_{2}(t)$ in Example 2 .

\section{Conclusions}

This article dealt with the global Mittag-Leffler stability and stabilization analysis of FOQVMNNs. The state feedback stabilizing control law is designed to stabilize the considered NNs. To solve this problem easily, by applying Hamilton rules in quaternion multiplication, the FOQVMNN is divided into four fractional-order real-valued FOQVMNNs. By using the method of Lyapunov fractional-order derivative, fractional-order differential inclusions, set-valued maps, several global Mittag-Leffler stability, and stabilization conditions of considered FOQVMNNs are established. The feasibility and advantages of the results obtained are illustrated in two numerical examples. We wish to point out that our main results will lead to the generalization of more QVNNs such as discrete-time QVNNs, stochastic QVNNs, and uncertain QVNNs. In the near future, we will focus on the robust stability of discrete-time QVNNs.

Author Contributions: Funding acquisition, P.C.; Conceptualization, G.R.; Software, P.C., P.K. and R.S.; Formal analysis, G.R.; Methodology, G.R.; Supervision, C.P.L.; Writing-original draft, G.R.; Validation, G.R.; Writing-review and editing, G.R. All authors have read and agreed to the published version of the manuscript.

Funding: This research was supported by Chiang Mai University.

Acknowledgments: This research was supported by Chiang Mai University.

Conflicts of Interest: The authors declare no conflict of interest. 


\section{Appendix A}

\section{Proof. Proof of Theorem 1}

We define the following Lyapunov function:

$$
\mathscr{V}\left(w_{x}(t)\right)=\sum_{x=1}^{4 n} \frac{1}{\varsigma}\left|w_{x}(t)\right|^{\varsigma}=\sum_{x=1}^{n} \frac{1}{\varsigma}\left|a_{x}(t)\right|^{\varsigma}+\sum_{x=1}^{n} \frac{1}{\varsigma}\left|b_{x}(t)\right|^{\varsigma}+\sum_{x=1}^{n} \frac{1}{\varsigma}\left|c_{x}(t)\right|^{\varsigma}+\sum_{x=1}^{n} \frac{1}{\varsigma}\left|d_{x}(t)\right|^{\varsigma} .
$$

From (7), it follows that

$$
\begin{aligned}
&{ }^{C} \mathcal{D}_{0, t}^{\sigma} \mathscr{V}\left(w_{x}(t)\right) \leq \sum_{x=1}^{n}\left|a_{x}(t)\right|^{\zeta-1}\left[-\mathbf{a}_{x}\left|a_{x}(t)\right|+\sum_{y=1}^{n} \tilde{\alpha}_{x y}^{R}\left|\mathscr{G}_{y}^{R}\right|-\sum_{y=1}^{n} \tilde{\alpha}_{x y}^{I}\left|\mathscr{G}_{y}^{I}\right|-\sum_{y=1}^{n} \tilde{\alpha}_{x y}^{J}\left|\mathscr{G}_{y}^{J}\right|\right. \\
&\left.-\sum_{y=1}^{n} \tilde{\alpha}_{x y}^{K}\left|\mathscr{G}_{y}^{K}\right|\right]+\sum_{x=1}^{n}\left|b_{x}(t)\right|^{\zeta-1}\left[-\mathbf{a}_{x}\left|b_{x}(t)\right|+\sum_{y=1}^{n} \tilde{\alpha}_{x y}^{R}\left|\mathscr{G}_{y}^{I}\right|+\sum_{y=1}^{n} \tilde{\alpha}_{x y}^{I}\left|\mathscr{G}_{y}^{R}\right|\right. \\
&\left.+\sum_{y=1}^{n} \tilde{\alpha}_{x y}^{J}\left|\mathscr{G}_{y}^{K}\right|-\sum_{y=1}^{n} \tilde{\alpha}_{x y}^{K}\left|\mathscr{G}_{y}^{J}\right|\right]+\sum_{x=1}^{n}\left|c_{x}(t)\right|^{\varsigma-1}\left[-\mathbf{a}_{x}\left|c_{x}(t)\right|+\sum_{y=1}^{n} \tilde{\alpha}_{x y}^{R}\left|\mathscr{G}_{y}^{J}\right|\right. \\
&\left.+\sum_{y=1}^{n} \tilde{\alpha}_{x y}^{J}\left|\mathscr{G}_{y}^{R}\right|+\sum_{y=1}^{n} \tilde{\alpha}_{x y}^{K}\left|\mathscr{G}_{y}^{I}\right|-\sum_{y=1}^{n} \tilde{\alpha}_{x y}^{I}\left|\mathscr{G}_{y}^{K}\right|\right]+\sum_{x=1}^{n}\left|d_{x}(t)\right|^{\zeta-1}\left[-\mathbf{a}_{x}\left|d_{x}(t)\right|\right. \\
&\left.+\sum_{y=1}^{n} \tilde{\alpha}_{x y}^{R}\left|\mathscr{G}_{y}^{K}\right|+\sum_{y=1}^{n} \tilde{\alpha}_{x y}^{I}\left|\mathscr{G}_{y}^{J}\right|+\sum_{y=1}^{n} \tilde{\alpha}_{x y}^{K}\left|\mathscr{G}_{y}^{R}\right|-\sum_{y=1}^{n} \tilde{\alpha}_{x y}^{J}\left|\mathscr{G}_{y}^{I}\right|\right] \\
&{ }^{C} \mathcal{D}_{0, t}^{\sigma} \mathscr{V}\left(w_{x}(t)\right) \leq \sum_{x=1}^{n}\left|a_{x}(t)\right|^{\varsigma-1}\left[-\mathbf{a}_{x}\left|a_{x}(t)\right|+\sum_{y=1}^{n} \tilde{\alpha}_{x y}^{R}\left(\mathscr{G}_{y}^{*}\right)-\sum_{y=1}^{n} \tilde{\alpha}_{x y}^{I}\left(\mathscr{G}_{y}^{* *}\right)-\sum_{y=1}^{n} \tilde{\alpha}_{x y}^{J}\left(\mathscr{G}_{y}^{* * *}\right)\right. \\
&\left.-\sum_{y=1}^{n} \tilde{\alpha}_{x y}^{K}\left(\mathscr{G}_{y}^{* * * *}\right)\right]+\sum_{x=1}^{n}\left|b_{x}(t)\right|^{\varsigma-1}\left[-\mathbf{a}_{x}\left|b_{x}(t)\right|+\sum_{y=1}^{n} \tilde{\alpha}_{x y}^{R}\left(\mathscr{G}_{y}^{* *}\right)+\sum_{y=1}^{n} \tilde{\alpha}_{x y}^{I}\left(\mathscr{G}_{y}^{*}\right)\right. \\
&\left.+\sum_{y=1}^{n} \tilde{\alpha}_{x y}^{J}\left(\mathscr{G}_{y}^{* * * *}\right)-\sum_{y=1}^{n} \tilde{\alpha}_{x y}^{K}\left(\mathscr{G}_{y}^{* * *}\right)\right]+\sum_{x=1}^{n}\left|c_{x}(t)\right|^{\varsigma-1}\left[-\mathbf{a}_{x}\left|c_{x}(t)\right|+\sum_{y=1}^{n} \tilde{\alpha}_{x y}^{R}\left(\mathscr{G}_{y}^{* * *}\right)\right. \\
&\left.+\sum_{y=1}^{n} \tilde{\alpha}_{x y}^{J}\left(\mathscr{G}_{y}^{*}\right)+\sum_{y=1}^{n} \tilde{\alpha}_{x y}^{K}\left(\mathscr{G}_{y}^{* *}\right)-\sum_{y=1}^{n} \tilde{\alpha}_{x y}^{I}\left(\mathscr{G}_{y}^{* * * *}\right)\right]+\sum_{x=1}^{n}\left|d_{x}(t)\right|^{\zeta-1}\left[-\mathbf{a}_{x}\left|d_{x}(t)\right|\right. \\
&\left.+\sum_{y=1}^{n} \tilde{\alpha}_{x y}^{R}\left(\mathscr{G}_{y}^{* * * *}\right)+\sum_{y=1}^{n} \tilde{\alpha}_{x y}^{I}\left(\mathscr{G}_{y}^{* * *}\right)+\sum_{y=1}^{n} \tilde{\alpha}_{x y}^{K}\left(\mathscr{G}_{y}^{*}\right)-\sum_{y=1}^{n} \tilde{\alpha}_{x y}^{J}\left(\mathscr{G}_{y}^{* *}\right)\right] \\
&
\end{aligned}
$$




$$
\begin{aligned}
& { }^{C} \mathcal{D}_{0, t}^{\sigma} \mathscr{V}\left(w_{x}(t)\right) \leq-\sum_{x=1}^{n} \mathbf{a}_{x}\left|a_{x}(t)\right|^{\varsigma}+\sum_{x=1}^{n} \sum_{y=1}^{n} \tilde{\alpha}_{x y}^{R} \wp_{y}^{R R}\left|a_{x}\right|^{*}\left|a_{y}\right|+\sum_{x=1}^{n} \sum_{y=1}^{n} \tilde{\alpha}_{x y}^{R} \wp_{y}^{R I}\left|a_{x}\right|^{*}\left|b_{y}\right| \\
& +\sum_{x=1}^{n} \sum_{y=1}^{n} \tilde{\alpha}_{x y}^{R} \wp_{y}^{R J}\left|a_{x}\right|^{*}\left|c_{y}\right|+\sum_{x=1}^{n} \sum_{y=1}^{n} \tilde{\alpha}_{x y}^{R} \wp_{y}^{R K}\left|a_{x}\right|^{*}\left|d_{y}\right|-\sum_{x=1}^{n} \sum_{y=1}^{n} \tilde{\alpha}_{x y}^{I} \wp_{y}^{I R}\left|a_{x}\right|^{*}\left|a_{y}\right| \\
& -\sum_{x=1}^{n} \sum_{y=1}^{n} \tilde{\alpha}_{x y}^{I} \wp_{y}^{I I}\left|a_{x}\right|^{*}\left|b_{y}\right|-\left.\sum_{x=1}^{n} \sum_{y=1}^{n} \tilde{\alpha}_{x y}^{I} \wp_{y}^{I J}\left|a_{x}(t)\right| a_{x}\right|^{*}\left|c_{y}\right|-\sum_{x=1}^{n} \sum_{y=1}^{n} \tilde{\alpha}_{x y}^{I} \wp_{y}^{I K}\left|a_{x}\right|^{*}\left|d_{y}\right| \\
& -\sum_{x=1}^{n} \sum_{y=1}^{n} \tilde{\alpha}_{x y}^{J} \wp_{y}^{J R}\left|a_{x}\right|^{*}\left|a_{y}\right|-\sum_{x=1}^{n} \sum_{y=1}^{n} \tilde{\alpha}_{x y}^{J} \wp_{y}^{J I}\left|a_{x}\right|^{*}\left|b_{y}\right|-\sum_{x=1}^{n} \sum_{y=1}^{n} \tilde{\alpha}_{x y}^{J} \wp_{y}^{J J}\left|a_{x}\right|^{*}\left|c_{y}\right| \\
& -\sum_{x=1}^{n} \sum_{y=1}^{n} \tilde{\alpha}_{x y}^{J} \wp_{y}^{J K}\left|a_{x}\right|^{*}\left|d_{y}\right|-\sum_{x=1}^{n} \sum_{y=1}^{n} \tilde{\alpha}_{x y}^{K} \wp_{y}^{K R}\left|a_{x}\right|^{*}\left|a_{y}\right|-\sum_{x=1}^{n} \sum_{y=1}^{n} \tilde{\alpha}_{x y}^{K} \wp_{y}^{K I}\left|a_{x}\right|^{*}\left|b_{y}\right| \\
& -\sum_{x=1}^{n} \sum_{y=1}^{n} \tilde{\alpha}_{x y}^{K} \wp_{y}^{K J}\left|a_{x}\right|^{*}\left|c_{y}\right|-\sum_{x=1}^{n} \sum_{y=1}^{n} \tilde{\alpha}_{x y}^{K} \wp_{y}^{K K}\left|a_{x}\right|^{*}\left|d_{y}\right|-\sum_{x=1}^{n} \mathbf{a}_{x}\left|a_{x}\right|^{*}\left|a_{y}\right|\left|b_{x}(t)\right|^{\varsigma} \\
& +\sum_{x=1}^{n} \sum_{y=1}^{n} \tilde{\alpha}_{x y}^{R} \wp_{y}^{I R}\left|b_{x}\right|^{*}\left|a_{y}\right|+\sum_{x=1}^{n} \sum_{y=1}^{n} \tilde{\alpha}_{x y}^{R} \wp_{y}^{I I}\left|b_{x}\right|^{*}\left|b_{y}\right|+\sum_{x=1}^{n} \sum_{y=1}^{n} \tilde{\alpha}_{x y}^{R} \wp_{y}^{I J}\left|b_{x}\right|^{*}\left|c_{y}\right| \\
& +\sum_{x=1}^{n} \sum_{y=1}^{n} \tilde{\alpha}_{x y}^{R} \wp_{y}^{I K}\left|b_{x}\right|^{*}\left|d_{y}\right|+\sum_{x=1}^{n} \sum_{y=1}^{n} \tilde{\alpha}_{x y}^{I} \wp_{y}^{R R}\left|b_{x}\right|^{*}\left|a_{y}\right|+\sum_{x=1}^{n} \sum_{y=1}^{n} \tilde{\alpha}_{x y}^{I} \wp_{y}^{R I}\left|b_{x}\right|^{*}\left|b_{y}\right| \\
& +\sum_{x=1}^{n} \sum_{y=1}^{n} \tilde{\alpha}_{x y}^{I} \wp_{y}^{R J}\left|b_{x}\right|^{*}\left|c_{y}\right|+\sum_{x=1}^{n} \sum_{y=1}^{n} \tilde{\alpha}_{x y}^{I} \wp_{y}^{R K}\left|b_{x}\right|^{*}\left|d_{y}\right|+\sum_{x=1}^{n} \sum_{y=1}^{n} \tilde{\alpha}_{x y}^{J} \wp_{y}^{K R}\left|b_{x}\right|^{*}\left|a_{y}\right| \\
& +\sum_{x=1}^{n} \sum_{y=1}^{n} \tilde{\alpha}_{x y}^{J} \wp_{y}^{K I}\left|b_{x}\right|^{*}\left|b_{y}\right|+\sum_{x=1}^{n} \sum_{y=1}^{n} \tilde{\alpha}_{x y}^{J} \wp_{y}^{K J}\left|b_{x}\right|^{*}\left|c_{y}\right|+\sum_{x=1}^{n} \sum_{y=1}^{n} \tilde{\alpha}_{x y}^{J} \wp_{y}^{K K}\left|b_{x}\right|^{*}\left|d_{y}\right| \\
& -\sum_{x=1}^{n} \sum_{y=1}^{n} \tilde{\alpha}_{x y}^{K} \wp_{y}^{J R}\left|b_{x}\right|^{*}\left|a_{y}\right|-\sum_{x=1}^{n} \sum_{y=1}^{n} \tilde{\alpha}_{x y}^{K} \wp_{y}^{J I}\left|b_{x}\right|^{*}\left|b_{y}\right|-\sum_{x=1}^{n} \sum_{y=1}^{n} \tilde{\alpha}_{x y}^{K} \wp_{y}^{J J}\left|b_{x}\right|^{*}\left|c_{y}\right| \\
& -\sum_{x=1}^{n} \sum_{y=1}^{n} \tilde{\alpha}_{x y}^{K} \wp_{y}^{J K}\left|b_{x}\right|^{*}\left|d_{y}\right|-\sum_{x=1}^{n} \mathbf{a}_{x}\left|c_{x}(t)\right|^{\varsigma}+\sum_{x=1}^{n} \sum_{y=1}^{n} \tilde{\alpha}_{x y}^{R} \wp_{y}^{J R}\left|c_{x}\right|^{*}\left|a_{y}\right| \\
& +\sum_{x=1}^{n} \sum_{y=1}^{n} \tilde{\alpha}_{x y}^{R} \wp_{y}^{J I}\left|c_{x}\right|^{*}\left|b_{y}\right|+\sum_{x=1}^{n} \sum_{y=1}^{n} \tilde{\alpha}_{x y}^{R} \wp_{y}^{J J}\left|c_{x}\right|^{*}\left|c_{y}\right|+\sum_{x=1}^{n} \sum_{y=1}^{n} \tilde{\alpha}_{x y}^{R} \wp_{y}^{J K}\left|c_{x}\right|^{*}\left|d_{y}\right| \\
& +\sum_{x=1}^{n} \sum_{y=1}^{n} \tilde{\alpha}_{x y}^{J} \wp_{y}^{R R}\left|c_{x}\right|^{*}\left|a_{y}\right|+\sum_{x=1}^{n} \sum_{y=1}^{n} \tilde{\alpha}_{x y}^{J} \wp_{y}^{R I}\left|c_{x}\right|^{*}\left|b_{y}\right|+\sum_{x=1}^{n} \sum_{y=1}^{n} \tilde{\alpha}_{x y}^{J} \wp_{y}^{R J}\left|c_{x}\right|^{*}\left|c_{y}\right| \\
& +\sum_{x=1}^{n} \sum_{y=1}^{n} \tilde{\alpha}_{x y}^{J} \wp_{y}^{R K}\left|c_{x}\right|^{*}\left|d_{y}\right|+\sum_{x=1}^{n} \sum_{y=1}^{n} \tilde{\alpha}_{x y}^{K} \wp_{y}^{I R}\left|c_{x}\right|^{*}\left|a_{y}\right|+\sum_{x=1}^{n} \sum_{y=1}^{n} \tilde{\alpha}_{x y}^{K} \wp_{y}^{I I}\left|c_{x}\right|^{*}\left|b_{y}\right| \\
& +\sum_{x=1}^{n} \sum_{y=1}^{n} \tilde{\alpha}_{x y}^{K} \wp_{y}^{I J}\left|c_{x}\right|^{*}\left|c_{y}\right|+\sum_{x=1}^{n} \sum_{y=1}^{n} \tilde{\alpha}_{x y}^{K} \wp_{y}^{I K}\left|c_{x}\right|^{*}\left|d_{y}\right|-\sum_{x=1}^{n} \sum_{y=1}^{n} \tilde{\alpha}_{x y}^{I} \wp_{y}^{K R}\left|c_{x}\right|^{*}\left|a_{y}\right| \\
& -\sum_{x=1}^{n} \sum_{y=1}^{n} \tilde{\alpha}_{x y}^{I} \wp_{y}^{K I}\left|c_{x}\right|^{*}\left|b_{y}\right|-\sum_{x=1}^{n} \sum_{y=1}^{n} \tilde{\alpha}_{x y}^{I} \wp_{y}^{K J}\left|c_{x}\right|^{*}\left|c_{y}\right|-\sum_{x=1}^{n} \sum_{y=1}^{n} \tilde{\alpha}_{x y}^{I} \wp_{y}^{K K}\left|c_{x}\right|^{*}\left|d_{y}\right|
\end{aligned}
$$




$$
\begin{aligned}
& -\sum_{x=1}^{n} \sum_{x=1}^{n} \mathbf{a}_{x}\left|d_{x}(t)\right|^{\varsigma}+\sum_{x=1}^{n} \sum_{y=1}^{n} \tilde{\alpha}_{x y}^{R} \wp_{y}^{K R}\left|d_{x}\right|^{*}\left|a_{y}\right|+\sum_{x=1}^{n} \sum_{y=1}^{n} \tilde{\alpha}_{x y}^{R} \wp_{y}^{K I}\left|d_{x}\right|^{*}\left|b_{y}\right| \\
& +\sum_{x=1}^{n} \sum_{y=1}^{n} \tilde{\alpha}_{x y}^{R} \wp_{y}^{K J}\left|d_{x}\right|^{*}\left|c_{y}\right|+\sum_{x=1}^{n} \sum_{y=1}^{n} \tilde{\alpha}_{x y}^{R} \wp_{y}^{K K}\left|d_{x}\right|^{*}\left|d_{y}\right|+\sum_{x=1}^{n} \sum_{y=1}^{n} \tilde{\alpha}_{x y}^{I} \wp_{y}^{J R}\left|d_{x}\right|^{*}\left|a_{y}\right| \\
& +\sum_{x=1}^{n} \sum_{y=1}^{n} \tilde{\alpha}_{x y}^{I} \wp_{y}^{J I}\left|d_{x}\right|^{*}\left|b_{y}\right|+\sum_{x=1}^{n} \sum_{y=1}^{n} \tilde{\alpha}_{x y}^{I} \wp_{y}^{J J}\left|d_{x}\right|^{*}\left|c_{y}\right|+\sum_{x=1}^{n} \sum_{y=1}^{n} \tilde{\alpha}_{x y}^{I} \wp_{y}^{J K}\left|d_{x}\right|^{*}\left|d_{y}\right| \\
& +\sum_{x=1}^{n} \sum_{y=1}^{n} \tilde{\alpha}_{x y}^{K} \wp_{y}^{R R}\left|d_{x}\right|^{*}\left|a_{y}\right|+\sum_{x=1}^{n} \sum_{y=1}^{n} \tilde{\alpha}_{x y}^{K} \wp_{y}^{R I}\left|d_{x}\right|^{*}\left|b_{y}\right|+\sum_{x=1}^{n} \sum_{y=1}^{n} \tilde{\alpha}_{x y}^{K} \wp_{y}^{R J}\left|d_{x}\right|^{*}\left|c_{y}\right| \\
& +\sum_{x=1}^{n} \sum_{y=1}^{n} \tilde{\alpha}_{x y}^{K} \wp_{y}^{R K}\left|d_{x}\right|^{*}\left|d_{y}\right|-\sum_{x=1}^{n} \sum_{y=1}^{n} \tilde{\alpha}_{x y}^{J} \wp_{y}^{I R}\left|d_{x}\right|^{*}\left|a_{y}\right|-\sum_{x=1}^{n} \sum_{y=1}^{n} \tilde{\alpha}_{x y}^{J} \wp_{y}^{I I}\left|d_{x}\right|^{*}\left|b_{y}\right| \\
& -\sum_{x=1}^{n} \sum_{y=1}^{n} \tilde{\alpha}_{x y}^{I} \wp_{y}^{I J}\left|d_{x}\right|^{*}\left|c_{y}\right|-\sum_{x=1}^{n} \sum_{y=1}^{n} \tilde{\alpha}_{x y}^{J} \wp_{y}^{I K}\left|d_{x}\right|^{*}\left|d_{y}\right| .
\end{aligned}
$$

By Lemma 1, it follows that

$$
\begin{aligned}
\left|a_{x}\right|^{*}\left|a_{y}\right| & \leq \frac{\varsigma-1}{\varsigma}\left[\left|a_{x}(t)\right|^{\varsigma-1} v_{1}^{\frac{\varsigma-1}{\zeta}}\right]^{\frac{\varsigma}{\zeta-1}}+\frac{1}{\zeta}\left[\left|a_{y}(t)\right| v_{1}^{-\frac{\varsigma-1}{\zeta}}\right]^{\varsigma} \\
& =\left|a^{*} a\right|_{y} .
\end{aligned}
$$

In the same way, we have

$$
\begin{aligned}
& \left|a_{x}\right|^{*}\left|b_{y}\right| \leq\left|a^{*} b\right|_{y}, \\
& \left|a_{x}\right|^{*}\left|c_{y}\right| \leq\left|a^{*} c\right|_{y,} \\
& \left|a_{x}\right|^{*}\left|d_{y}\right| \leq\left|a^{*} d\right|_{y,} \\
& \left|b_{x}\right|^{*}\left|a_{y}\right| \leq\left|b^{*} a\right|_{y,} \\
& \left|b_{x}\right|^{*}\left|b_{y}\right| \leq\left|b^{*} b\right|_{y}, \\
& \left|b_{x}\right|^{*}\left|c_{y}\right| \leq\left|b^{*} c\right|_{y,} \\
& \left|b_{x}\right|^{*}\left|d_{y}\right| \leq\left|b^{*} d\right|_{y}, \\
& \left|c_{x}\right|^{*}\left|a_{y}\right| \leq\left|c^{*} a\right|_{y}, \\
& \left|c_{x}\right|^{*}\left|b_{y}\right| \leq\left|c^{*} b\right|_{y}, \\
& \left|c_{x}\right|^{*}\left|c_{y}\right| \leq\left|c^{*} c\right|_{y,} \\
& \left|c_{x}\right|^{*}\left|d_{y}\right| \leq\left|c^{*} d\right|_{y}, \\
& \left|d_{x}\right|^{*}\left|a_{y}\right| \leq\left|d^{*} a\right|_{y}, \\
& \left|d_{x}\right|^{*}\left|b_{y}\right| \leq\left|d^{*} b\right|_{y,} \\
& \left|d_{x}\right|^{*}\left|c_{y}\right| \leq\left|d^{*} c\right|_{y,} \\
& \left|d_{x}\right|^{*}\left|d_{y}\right| \leq\left|d^{*} d\right|_{y} .
\end{aligned}
$$


Substituting (A5)-(A20) into (A4), then

$$
\begin{aligned}
& { }^{C} \mathcal{D}_{0, t}^{\sigma} \mathscr{V}\left(w_{x}(t)\right) \leq-\sum_{x=1}^{n} \mathbf{a}_{x}\left|a_{x}(t)\right|^{\varsigma}+\sum_{x=1}^{n} \sum_{y=1}^{n} \tilde{\alpha}_{x y}^{R} \wp_{y}^{R R}\left|a^{*} a\right|_{y}+\sum_{x=1}^{n} \sum_{y=1}^{n} \tilde{\alpha}_{x y}^{R} \wp_{y}^{R I}\left|a^{*} b\right|_{y}+\sum_{x=1}^{n} \sum_{y=1}^{n} \tilde{\alpha}_{x y}^{R} \wp_{y}^{R J}\left|a^{*} c\right|_{y} \\
& +\sum_{x=1}^{n} \sum_{y=1}^{n} \tilde{\alpha}_{x y}^{R} \wp_{y}^{R K}\left|a^{*} d\right|_{y}-\sum_{x=1}^{n} \sum_{y=1}^{n} \tilde{\alpha}_{x y}^{I} \wp_{y}^{I R}\left|a^{*} a\right|_{y}-\sum_{x=1}^{n} \sum_{y=1}^{n} \tilde{\alpha}_{x y}^{I} \wp_{y}^{I I}\left|a^{*} b\right|_{y}-\sum_{x=1}^{n} \sum_{y=1}^{n} \tilde{\alpha}_{x y}^{I} \wp_{y}^{I I}\left|a^{*} c\right|_{y} \\
& -\sum_{x=1}^{n} \sum_{y=1}^{n} \tilde{\alpha}_{x y}^{I} \wp_{y}^{I K}\left|a^{*} d\right|_{y}-\sum_{x=1}^{n} \sum_{y=1}^{n} \tilde{\alpha}_{x y}^{J} \wp_{y}^{J R}\left|a^{*} a\right|_{y}-\sum_{x=1}^{n} \sum_{y=1}^{n} \tilde{\alpha}_{x y}^{J} \wp_{y}^{J I}\left|a^{*} b\right|_{y}-\sum_{x=1}^{n} \sum_{y=1}^{n} \tilde{\alpha}_{x y}^{J} \wp_{y}^{J J}\left|a^{*} c\right|_{y} \\
& -\sum_{x=1}^{n} \sum_{y=1}^{n} \tilde{\alpha}_{x y}^{J} \wp_{y}^{J K}\left|a^{*} d\right|_{y}-\sum_{x=1}^{n} \sum_{y=1}^{n} \tilde{\alpha}_{x y}^{K} \wp_{y}^{K R}\left|a^{*} a\right|_{y}-\sum_{x=1}^{n} \sum_{y=1}^{n} \tilde{\alpha}_{x y}^{K} \wp_{y}^{K I}\left|a^{*} b\right|_{y}-\sum_{x=1}^{n} \sum_{y=1}^{n} \tilde{\alpha}_{x y}^{K} \wp_{y}^{K J}\left|a^{*} c\right|_{y} \\
& -\sum_{x=1}^{n} \sum_{y=1}^{n} \tilde{\alpha}_{x y}^{K} \wp_{y}^{K K}\left|a^{*} d\right|_{y}-\sum_{x=1}^{n} \mathbf{a}_{x}\left|b_{x}(t)\right|^{\varsigma}+\sum_{x=1}^{n} \sum_{y=1}^{n} \tilde{\alpha}_{x y}^{R} \wp_{y}^{I R}\left|b^{*} a\right|_{y}+\sum_{x=1}^{n} \sum_{y=1}^{n} \tilde{\alpha}_{x y}^{R} \wp_{y}^{I I}\left|b^{*} b\right|_{y} \\
& +\sum_{x=1}^{n} \sum_{y=1}^{n} \tilde{\alpha}_{x y}^{R} \wp_{y}^{I I}\left|b^{*} c\right|_{y}+\sum_{x=1}^{n} \sum_{y=1}^{n} \tilde{\alpha}_{x y}^{R} \wp_{y}^{I K}\left|b^{*} d\right|_{y}+\sum_{x=1}^{n} \sum_{y=1}^{n} \tilde{\alpha}_{x y}^{I} \wp_{y}^{R R}\left|b^{*} a\right|_{y}+\sum_{x=1}^{n} \sum_{y=1}^{n} \tilde{\alpha}_{x y}^{I} \wp_{y}^{R I}\left|b^{*} b\right|_{y} \\
& +\sum_{x=1}^{n} \sum_{y=1}^{n} \tilde{\alpha}_{x y}^{I} \wp_{y}^{R J}\left|b^{*} c\right|_{y}+\sum_{x=1}^{n} \sum_{y=1}^{n} \tilde{\alpha}_{x y}^{I} \wp_{y}^{R K}\left|b^{*} d\right|_{y}+\sum_{x=1}^{n} \sum_{y=1}^{n} \tilde{\alpha}_{x y}^{J} \wp_{y}^{K R}\left|b^{*} a\right|_{y}+\sum_{x=1}^{n} \sum_{y=1}^{n} \tilde{\alpha}_{x y}^{J} \wp_{y}^{K I}\left|b^{*} b\right|_{y} \\
& +\sum_{x=1}^{n} \sum_{y=1}^{n} \tilde{\alpha}_{x y}^{J} \wp_{y}^{K J}\left|b^{*} c\right|_{y}+\sum_{x=1}^{n} \sum_{y=1}^{n} \tilde{\alpha}_{x y}^{J} \wp_{y}^{K K}\left|b^{*} d\right|_{y}-\sum_{x=1}^{n} \sum_{y=1}^{n} \tilde{\alpha}_{x y}^{K} \wp_{y}^{J R}\left|b^{*} a\right|_{y}-\sum_{x=1}^{n} \sum_{y=1}^{n} \tilde{\alpha}_{x y}^{K} \wp_{y}^{J I}\left|b^{*} b\right|_{y} \\
& -\sum_{x=1}^{n} \sum_{y=1}^{n} \tilde{\alpha}_{x y}^{K} \wp_{y}^{J J}\left|b^{*} c\right|_{y}-\sum_{x=1}^{n} \sum_{y=1}^{n} \tilde{\alpha}_{x y}^{K} \wp_{y}^{J K}\left|b^{*} d\right|_{y}-\sum_{x=1}^{n} \mathbf{a}_{x}\left|c_{x}(t)\right|^{\varsigma}+\sum_{x=1}^{n} \sum_{y=1}^{n} \tilde{\alpha}_{x y}^{R} \wp_{y}^{J R}\left|b^{*} a\right|_{y} \\
& +\sum_{x=1}^{n} \sum_{y=1}^{n} \tilde{\alpha}_{x y}^{R} \wp_{y}^{J I}\left|b^{*} b\right|_{y}+\sum_{x=1}^{n} \sum_{y=1}^{n} \tilde{\alpha}_{x y}^{R} \wp_{y}^{J J}\left|b^{*} c\right|_{y}+\sum_{x=1}^{n} \sum_{y=1}^{n} \tilde{\alpha}_{x y}^{R} \wp_{y}^{J K}\left|b^{*} d\right|_{y}+\sum_{x=1}^{n} \sum_{y=1}^{n} \tilde{\alpha}_{x y}^{J} \wp_{y}^{R R}\left|b^{*} a\right|_{y} \\
& +\sum_{x=1}^{n} \sum_{y=1}^{n} \tilde{\alpha}_{x y}^{J} \wp_{y}^{R I}\left|b^{*} b\right|_{y}+\sum_{x=1}^{n} \sum_{y=1}^{n} \tilde{\alpha}_{x y}^{J} \wp_{y}^{R J}\left|b^{*} c\right|_{y}+\sum_{x=1}^{n} \sum_{y=1}^{n} \tilde{\alpha}_{x y}^{J} \wp_{y}^{R K}\left|b^{*} d\right|_{y}+\sum_{x=1}^{n} \sum_{y=1}^{n} \tilde{\alpha}_{x y}^{K} \wp_{y}^{I R}\left|c^{*} a\right|_{y} \\
& +\sum_{x=1}^{n} \sum_{y=1}^{n} \tilde{\alpha}_{x y}^{K} \wp_{y}^{I I}\left|c^{*} b\right|_{y}+\sum_{x=1}^{n} \sum_{y=1}^{n} \tilde{\alpha}_{x y}^{K} \wp_{y}^{I J}\left|c^{*} c\right|_{y}+\sum_{x=1}^{n} \sum_{y=1}^{n} \tilde{\alpha}_{x y}^{K} \wp_{y}^{I K}\left|c^{*} d\right|_{y}-\sum_{x=1}^{n} \sum_{y=1}^{n} \tilde{\alpha}_{x y}^{I} \wp_{y}^{K R}\left|c^{*} a\right|_{y} \\
& -\sum_{x=1}^{n} \sum_{y=1}^{n} \tilde{\alpha}_{x y}^{I} \wp_{y}^{K I}\left|c^{*} b\right|_{y}-\sum_{x=1}^{n} \sum_{y=1}^{n} \tilde{\alpha}_{x y}^{I} \wp_{y}^{K J}\left|c^{*} c\right|_{y}-\sum_{x=1}^{n} \sum_{y=1}^{n} \tilde{\alpha}_{x y}^{I} \wp_{y}^{K K}\left|c^{*} d\right|_{y}-\sum_{x=1}^{n} \sum_{x=1}^{n} \mathbf{a}_{x}\left|d_{x}(t)\right|^{\varsigma} \\
& +\sum_{x=1}^{n} \sum_{y=1}^{n} \tilde{\alpha}_{x y}^{R} \wp_{y}^{K R}\left|d^{*} a\right|_{y}+\sum_{x=1}^{n} \sum_{y=1}^{n} \tilde{\alpha}_{x y}^{R} \wp_{y}^{K I}\left|d^{*} b\right|_{y}+\sum_{x=1}^{n} \sum_{y=1}^{n} \tilde{\alpha}_{x y}^{R} \wp_{y}^{K J}\left|d^{*} c\right|_{y}+\sum_{x=1}^{n} \sum_{y=1}^{n} \tilde{\alpha}_{x y}^{R} \wp_{y}^{K K}\left|d^{*} d\right|_{y} \\
& +\sum_{x=1}^{n} \sum_{y=1}^{n} \tilde{\alpha}_{x y}^{I} \wp_{y}^{J R}\left|d^{*} a\right|_{y}+\sum_{x=1}^{n} \sum_{y=1}^{n} \tilde{\alpha}_{x y}^{I} \wp_{y}^{I I}\left|d^{*} b\right|_{y}+\sum_{x=1}^{n} \sum_{y=1}^{n} \tilde{\alpha}_{x y}^{I} \wp_{y}^{J J}\left|d^{*} c\right|_{y}+\sum_{x=1}^{n} \sum_{y=1}^{n} \tilde{\alpha}_{x y}^{I} \wp_{y}^{J K}\left|d^{*} d\right|_{y} \\
& +\sum_{x=1}^{n} \sum_{y=1}^{n} \tilde{\alpha}_{x y}^{K} \wp_{y}^{R R}\left|d^{*} a\right|_{y}+\sum_{x=1}^{n} \sum_{y=1}^{n} \tilde{\alpha}_{x y}^{K} \wp_{y}^{R I}\left|d^{*} b\right|_{y}+\sum_{x=1}^{n} \sum_{y=1}^{n} \tilde{\alpha}_{x y}^{K} \wp_{y}^{R J}\left|d^{*} c\right|_{y}+\sum_{x=1}^{n} \sum_{y=1}^{n} \tilde{\alpha}_{x y}^{K} \wp_{y}^{R K}\left|d^{*} d\right|_{y} \\
& -\sum_{x=1}^{n} \sum_{y=1}^{n} \tilde{\alpha}_{x y}^{J} \wp_{y}^{I R}\left|d^{*} a\right|_{y}-\sum_{x=1}^{n} \sum_{y=1}^{n} \tilde{\alpha}_{x y}^{I} \wp_{y}^{I I}\left|d^{*} b\right|_{y}-\sum_{x=1}^{n} \sum_{y=1}^{n} \tilde{\alpha}_{x y}^{J} \wp_{y}^{I J}\left|d^{*} c\right|_{y}-\sum_{x=1}^{n} \sum_{y=1}^{n} \tilde{\alpha}_{x y}^{I} \wp_{y}^{I K}\left|d^{*} d\right|_{y} .
\end{aligned}
$$




$$
\begin{aligned}
& =\sum_{x=1}^{n}\left\{-\mathbf{a}_{x} \varsigma+\sum_{y=1}^{n} \tilde{\alpha}_{x y}^{R} \wp_{y}^{R R}(\varsigma-1) v_{1}+\sum_{y=1}^{n} \tilde{\alpha}_{y x}^{R} \wp_{x}^{R R} \frac{1}{v_{1}^{\varsigma-1}}+\sum_{y=1}^{n} \tilde{\alpha}_{x y}^{R} \wp_{y}^{R I}(\varsigma-1) v_{2}\right. \\
& +\sum_{y=1}^{n} \tilde{\alpha}_{x y}^{R} \wp_{y}^{R J}(\varsigma-1) v_{3}+\sum_{y=1}^{n} \tilde{\alpha}_{x y}^{R} \wp_{y}^{R K}(\varsigma-1) v_{4}-\sum_{y=1}^{n} \tilde{\alpha}_{x y}^{I} \wp_{y}^{I R}(\varsigma-1) v_{1}-\sum_{y=1}^{n} \tilde{\alpha}_{y x}^{I} \wp_{x}^{I R} \frac{1}{v_{1}^{\zeta-1}} \\
& -\sum_{y=1}^{n} \tilde{\alpha}_{x y}^{I} \wp_{y}^{I I}(\varsigma-1) v_{2}-\sum_{y=1}^{n} \tilde{\alpha}_{x y}^{I} \wp_{y}^{I J}(\varsigma-1) v_{3}-\sum_{y=1}^{n} \tilde{\alpha}_{x y}^{I} \wp_{y}^{I K}(\varsigma-1) v_{4}-\sum_{y=1}^{n} \tilde{\alpha}_{x y}^{I} \wp_{y}^{J R}(\varsigma-1) v_{1} \\
& -\sum_{y=1}^{n} \tilde{\alpha}_{y x}^{J} \wp_{x}^{J R} \frac{1}{v_{1}^{\varsigma-1}}-\sum_{y=1}^{n} \tilde{\alpha}_{x y}^{J} \wp_{y}^{J I}(\varsigma-1) v_{2}-\sum_{y=1}^{n} \tilde{\alpha}_{x y}^{J} \wp_{y}^{J J}(\varsigma-1) v_{3}-\sum_{y=1}^{n} \tilde{\alpha}_{x y}^{J} \wp_{y}^{J K}(\varsigma-1) v_{4} \\
& -\sum_{y=1}^{n} \tilde{\alpha}_{x y}^{K} \wp_{y}^{K R}(\varsigma-1) v_{1}-\sum_{y=1}^{n} \tilde{\alpha}_{y x}^{K} \wp_{x}^{K R} \frac{1}{v_{1}^{\varsigma-1}}-\sum_{y=1}^{n} \tilde{\alpha}_{x y}^{K} \wp_{y}^{K I}(\varsigma-1) v_{2}-\sum_{y=1}^{n} \tilde{\alpha}_{x y}^{K} \wp_{y}^{K J}(\varsigma-1) v_{3} \\
& -\sum_{y=1}^{n} \tilde{\alpha}_{x y}^{K} \wp_{y}^{K K}(\varsigma-1) v_{4}+\sum_{y=1}^{n} \tilde{\alpha}_{y x}^{R} \wp_{x}^{I R} \frac{1}{v_{5}^{\varsigma-1}}+\sum_{y=1}^{n} \tilde{\alpha}_{y x}^{I} \wp_{x}^{R R} \frac{1}{v_{5}^{\varsigma-1}}+\sum_{y=1}^{n} \tilde{\alpha}_{y x}^{J} \wp_{x}^{K R} \frac{1}{v_{5}^{\varsigma-1}}-\sum_{y=1}^{n} \tilde{\alpha}_{y x}^{K} \wp_{x}^{J R} \frac{1}{v_{5}^{\varsigma-1}} \\
& +\sum_{y=1}^{n} \tilde{\alpha}_{y x}^{R} \wp_{x}^{J R} \frac{1}{v_{9}^{\varsigma-1}}+\sum_{y=1}^{n} \tilde{\alpha}_{y x}^{J} \wp_{x}^{R R} \frac{1}{v_{9}^{\varsigma-1}}+\sum_{y=1}^{n} \tilde{\alpha}_{y x}^{K} \wp_{x}^{I R} \frac{1}{v_{9}^{\varsigma-1}}-\sum_{y=1}^{n} \tilde{\alpha}_{y x}^{I} \wp_{x}^{K R} \frac{1}{v_{9}^{\varsigma-1}}+\sum_{y=1}^{n} \tilde{\alpha}_{y x}^{R} \wp_{x}^{K R} \frac{1}{v_{13}^{\varsigma-1}} \\
& \left.+\sum_{y=1}^{n} \tilde{\alpha}_{y x}^{I} \wp_{x}^{J R} \frac{1}{v_{13}^{\varsigma-1}}+\sum_{y=1}^{n} \tilde{\alpha}_{y x}^{K} \wp_{x}^{R R} \frac{1}{v_{13}^{\varsigma-1}}-\sum_{y=1}^{n} \tilde{\alpha}_{y x}^{J} \wp_{x}^{I R} \frac{1}{v_{13}^{S-1}}\right\} \frac{\left|a_{x}(t)\right|^{\varsigma}}{\varsigma} \\
& +\sum_{x=1}^{n}\left\{-\mathbf{a}_{x} \varsigma+\sum_{y=1}^{n} \tilde{\alpha}_{y x}^{R} \wp_{x}^{R I} \frac{1}{v_{2}^{\zeta-1}}-\sum_{y=1}^{n} \tilde{\alpha}_{y x}^{I} \wp_{x}^{I I} \frac{1}{v_{2}^{\zeta-1}}-\sum_{y=1}^{n} \tilde{\alpha}_{y x}^{I} \wp_{x}^{J I} \frac{1}{v_{2}^{\zeta-1}}-\sum_{y=1}^{n} \tilde{\alpha}_{y x}^{K} \wp_{x}^{K I} \frac{1}{v_{2}^{\zeta-1}}\right. \\
& +\sum_{y=1}^{n} \tilde{\alpha}_{x y}^{R} \wp_{y}^{I R}(\varsigma-1) v_{5}+\sum_{y=1}^{n} \tilde{\alpha}_{x y}^{R} \wp_{y}^{I I}(\varsigma-1) v_{6}+\sum_{y=1}^{n} \tilde{\alpha}_{y x}^{R} \wp_{x}^{I I} \frac{1}{v_{6}^{\varsigma-1}}+\sum_{y=1}^{n} \tilde{\alpha}_{x y}^{R} \wp_{y}^{I J}(\varsigma-1) v_{7} \\
& +\sum_{y=1}^{n} \tilde{\alpha}_{x y}^{R} \wp_{y}^{I K}(\varsigma-1) v_{8}+\sum_{y=1}^{n} \tilde{\alpha}_{x y}^{I} \wp_{y}^{R R}(\varsigma-1) v_{5}+\sum_{y=1}^{n} \tilde{\alpha}_{x y}^{I} \wp_{y}^{R I}(\varsigma-1) v_{6}+\sum_{y=1}^{n} \tilde{\alpha}_{y x}^{I} \wp_{x}^{R I} \frac{1}{v_{6}^{\varsigma-1}} \\
& +\sum_{y=1}^{n} \tilde{\alpha}_{x y}^{I} \wp_{y}^{R J}(\varsigma-1) v_{7}+\sum_{y=1}^{n} \tilde{\alpha}_{x y}^{I} \wp_{y}^{R K}(\varsigma-1) v_{8}+\sum_{y=1}^{n} \tilde{\alpha}_{x y}^{I} \wp_{y}^{K R}(\varsigma-1) v_{5}+\sum_{y=1}^{n} \tilde{\alpha}_{x y}^{I} \wp_{y}^{R I}(\varsigma-1) v_{6} \\
& +\sum_{y=1}^{n} \tilde{\alpha}_{y x}^{J} \wp_{x}^{K I} \frac{1}{v_{6}^{\varsigma-1}}+\sum_{y=1}^{n} \tilde{\alpha}_{x y}^{J} \wp_{y}^{K J}(\varsigma-1) v_{7}+\sum_{y=1}^{n} \tilde{\alpha}_{x y}^{J} \wp_{y}^{K K}(\varsigma-1) v_{8}-\sum_{y=1}^{n} \tilde{\alpha}_{x y}^{K} \wp_{y}^{J R}(\varsigma-1) v_{5} \\
& -\sum_{y=1}^{n} \tilde{\alpha}_{x y}^{K} \wp_{y}^{J I}(\varsigma-1) v_{6}-\sum_{y=1}^{n} \tilde{\alpha}_{y x}^{K} \wp_{x}^{J I} \frac{1}{v_{6}^{\varsigma-1}}-\sum_{y=1}^{n} \tilde{\alpha}_{x y}^{K} \wp_{y}^{J J}(\varsigma-1) v_{7}-\sum_{y=1}^{n} \tilde{\alpha}_{x y}^{K} \wp_{y}^{J K}(\varsigma-1) v_{8}
\end{aligned}
$$




$$
\begin{aligned}
& +\sum_{y=1}^{n} \tilde{\alpha}_{y x}^{R} \wp_{x}^{J I} \frac{1}{v_{10}^{\varsigma-1}}+\sum_{y=1}^{n} \tilde{\alpha}_{y x}^{J} \wp_{x}^{R I} \frac{1}{v_{10}^{\varsigma-1}}+\sum_{y=1}^{n} \tilde{\alpha}_{y x}^{K} \wp_{x}^{I I} \frac{1}{v_{10}^{\varsigma-1}}-\sum_{y=1}^{n} \tilde{\alpha}_{y x}^{I} \wp_{x}^{K I} \frac{1}{v_{10}^{\varsigma-1}} \\
& \left.+\sum_{y=1}^{n} \tilde{\alpha}_{y x}^{R} \wp_{x}^{K I} \frac{1}{v_{14}^{\varsigma-1}}+\sum_{y=1}^{n} \tilde{\alpha}_{y x}^{I} \wp_{x}^{J I} \frac{1}{v_{14}^{\varsigma-1}}+\sum_{y=1}^{n} \tilde{\alpha}_{y x}^{K} \wp_{x}^{R I} \frac{1}{v_{14}^{\varsigma-1}}+\sum_{y=1}^{n} \tilde{\alpha}_{y x}^{J} \wp_{x}^{I I} \frac{1}{v_{14}^{\varsigma-1}}\right\} \frac{\left|b_{x}(t)\right|^{\varsigma}}{\varsigma} \\
& +\sum_{x=1}^{n}\left\{-\mathbf{a}_{x} \varsigma+\sum_{y=1}^{n} \tilde{\alpha}_{y x}^{R} \wp_{x}^{R J} \frac{1}{v_{3}^{\varsigma-1}}-\sum_{y=1}^{n} \tilde{\alpha}_{y x}^{I} \wp_{x}^{I J} \frac{1}{v_{3}^{\varsigma-1}}-\sum_{y=1}^{n} \tilde{\alpha}_{y x}^{J} \wp_{x}^{J J} \frac{1}{v_{3}^{\varsigma-1}}-\sum_{y=1}^{n} \tilde{\alpha}_{y x}^{K} \wp_{x}^{K J} \frac{1}{v_{3}^{\varsigma-1}}\right. \\
& +\sum_{y=1}^{n} \tilde{\alpha}_{y x}^{R} \wp_{x}^{I J} \frac{1}{v_{7}^{\varsigma-1}}+\sum_{y=1}^{n} \tilde{\alpha}_{y x}^{I} \wp_{x}^{R J} \frac{1}{v_{7}^{\varsigma-1}}+\sum_{y=1}^{n} \tilde{\alpha}_{y x}^{J} \wp_{x}^{K J} \frac{1}{v_{7}^{\varsigma-1}}-\sum_{y=1}^{n} \tilde{\alpha}_{y x}^{K} \wp_{x}^{J J} \frac{1}{v_{7}^{\varsigma-1}}+\sum_{y=1}^{n} \tilde{\alpha}_{x y}^{R} \wp_{y}^{J R}(\varsigma-1) v_{9} \\
& +\sum_{y=1}^{n} \tilde{\alpha}_{x y}^{R} \wp_{y}^{J I}(\varsigma-1) v_{10}+\sum_{y=1}^{n} \tilde{\alpha}_{x y}^{R} \wp_{y}^{J J}(\varsigma-1) v_{11}+\sum_{y=1}^{n} \tilde{\alpha}_{y x}^{R} \wp_{x}^{J J} \frac{1}{v_{11}^{s-1}}+\sum_{y=1}^{n} \tilde{\alpha}_{x y}^{R} \wp_{y}^{J K}(\varsigma-1) v_{12} \\
& +\sum_{y=1}^{n} \tilde{\alpha}_{x y}^{J} \wp_{y}^{R R}(\varsigma-1) v_{9}+\sum_{y=1}^{n} \tilde{\alpha}_{x y}^{J} \wp_{y}^{R I}(\varsigma-1) v_{10}+\sum_{y=1}^{n} \tilde{\alpha}_{x y}^{J} \wp_{y}^{R J}(\varsigma-1) v_{11}+\sum_{y=1}^{n} \tilde{\alpha}_{y x}^{J} \wp_{x}^{R J} \frac{1}{v_{11}^{\varsigma-1}} \\
& +\sum_{y=1}^{n} \tilde{\alpha}_{x y}^{I} \wp_{y}^{R K}(\varsigma-1) v_{12}+\sum_{y=1}^{n} \tilde{\alpha}_{x y}^{K} \wp_{y}^{I R}(\varsigma-1) v_{9}+\sum_{y=1}^{n} \tilde{\alpha}_{x y}^{K} \wp_{y}^{I I}(\varsigma-1) v_{10}+\sum_{y=1}^{n} \tilde{\alpha}_{x y}^{K} \wp_{y}^{I J}(\varsigma-1) v_{11} \\
& +\sum_{y=1}^{n} \tilde{\alpha}_{y x}^{K} \wp_{x}^{I J} \frac{1}{v_{11}^{S-1}}+\sum_{y=1}^{n} \tilde{\alpha}_{x y}^{K} \wp_{y}^{I K}(\varsigma-1) v_{12}-\sum_{y=1}^{n} \tilde{\alpha}_{x y}^{I} \wp_{y}^{K R}(\varsigma-1) v_{9}-\sum_{y=1}^{n} \tilde{\alpha}_{x y}^{I} \wp_{y}^{K I}(\varsigma-1) v_{10} \\
& -\sum_{y=1}^{n} \tilde{\alpha}_{x y}^{I} \wp_{y}^{K J}(\varsigma-1) v_{11}-\sum_{y=1}^{n} \tilde{\alpha}_{y x}^{I} \wp_{x}^{K J} \frac{1}{v_{11}^{\varsigma-1}}-\sum_{y=1}^{n} \tilde{\alpha}_{x y}^{I} \wp_{y}^{K K}(\varsigma-1) v_{12}+\sum_{y=1}^{n} \tilde{\alpha}_{y x}^{R} \wp_{x}^{K J} \frac{1}{v_{15}^{\varsigma-1}} \\
& \left.+\sum_{y=1}^{n} \tilde{\alpha}_{y x}^{I} \wp_{x}^{J J} \frac{1}{v_{15}^{\varsigma-1}}+\sum_{y=1}^{n} \tilde{\alpha}_{y x}^{K} \wp_{x}^{R J} \frac{1}{v_{15}^{\varsigma-1}}-\sum_{y=1}^{n} \tilde{\alpha}_{y x}^{J} \wp_{x}^{I J} \frac{1}{v_{15}^{\varsigma-1}}\right\} \frac{\left|c_{x}(t)\right|^{\varsigma}}{\varsigma} \\
& +\sum_{x=1}^{n}\left\{-\mathbf{a}_{x} \varsigma+\sum_{y=1}^{n} \tilde{\alpha}_{y x}^{R} \wp_{x}^{R K} \frac{1}{v_{4}^{\varsigma-1}}-\sum_{y=1}^{n} \tilde{\alpha}_{y x}^{I} \wp_{x}^{I K} \frac{1}{v_{4}^{\varsigma-1}}-\sum_{y=1}^{n} \tilde{\alpha}_{y x}^{J} \wp_{x}^{J K} \frac{1}{v_{4}^{\varsigma-1}}-\sum_{y=1}^{n} \tilde{\alpha}_{y x}^{K} \wp_{x}^{K K} \frac{1}{v_{4}^{\varsigma-1}}\right. \\
& +\sum_{y=1}^{n} \tilde{\alpha}_{y x}^{R} \wp_{x}^{I K} \frac{1}{v_{8}^{\varsigma-1}}+\sum_{y=1}^{n} \tilde{\alpha}_{y x}^{I} \wp_{x}^{R K} \frac{1}{v_{8}^{S-1}}+\sum_{y=1}^{n} \tilde{\alpha}_{y x}^{J} \wp_{x}^{K K} \frac{1}{v_{8}^{\varsigma-1}}-\sum_{y=1}^{n} \tilde{\alpha}_{y x}^{K} \wp_{x}^{J K} \frac{1}{v_{8}^{S-1}}+\sum_{y=1}^{n} \tilde{\alpha}_{y x}^{R} \wp_{x}^{J K} \frac{1}{v_{12}^{\varsigma-1}} \\
& +\sum_{y=1}^{n} \tilde{\alpha}_{y x}^{J} \wp_{x}^{R K} \frac{1}{v_{12}^{\varsigma-1}}+\sum_{y=1}^{n} \tilde{\alpha}_{y x}^{K} \wp_{x}^{I K} \frac{1}{v_{12}^{\varsigma-1}}-\sum_{y=1}^{n} \tilde{\alpha}_{y x}^{I} \wp_{x}^{K K} \frac{1}{v_{12}^{\varsigma-1}}+\sum_{y=1}^{n} \tilde{\alpha}_{x y}^{R} \wp_{y}^{K R}(\varsigma-1) v_{13} \\
& +\sum_{y=1}^{n} \tilde{\alpha}_{x y}^{R} \wp_{y}^{K I}(\varsigma-1) v_{14}+\sum_{y=1}^{n} \tilde{\alpha}_{x y}^{R} \wp_{y}^{K J}(\varsigma-1) v_{15}+\sum_{y=1}^{n} \tilde{\alpha}_{x y}^{R} \wp_{y}^{K K}(\varsigma-1) v_{16}+\sum_{y=1}^{n} \tilde{\alpha}_{y x}^{R} \wp_{x}^{K K} \frac{1}{v_{16}^{\varsigma-1}} \\
& +\sum_{y=1}^{n} \tilde{\alpha}_{x y}^{I} \wp_{y}^{J R}(\varsigma-1) v_{13}+\sum_{y=1}^{n} \tilde{\alpha}_{x y}^{I} \wp_{y}^{J I}(\varsigma-1) v_{14}+\sum_{y=1}^{n} \tilde{\alpha}_{x y}^{I} \wp_{y}^{J J}(\varsigma-1) v_{15}+\sum_{y=1}^{n} \tilde{\alpha}_{x y}^{I} \wp_{y}^{J K}(\varsigma-1) v_{16} \\
& +\sum_{y=1}^{n} \tilde{\alpha}_{y x}^{I} \wp_{x}^{J K} \frac{1}{v_{16}^{\varsigma-1}}+\sum_{y=1}^{n} \tilde{\alpha}_{x y}^{K} \wp_{y}^{R R}(\varsigma-1) v_{13}+\sum_{y=1}^{n} \tilde{\alpha}_{x y}^{K} \wp_{y}^{R I}(\varsigma-1) v_{14}+\sum_{y=1}^{n} \tilde{\alpha}_{x y}^{K} \wp_{y}^{R J}(\varsigma-1) v_{15} \\
& +\sum_{y=1}^{n} \tilde{\alpha}_{x y}^{K} \wp_{y}^{R K}(\varsigma-1) v_{16}+\sum_{y=1}^{n} \tilde{\alpha}_{y x}^{K} \wp_{x}^{R K} \frac{1}{v_{16}^{\varsigma-1}}-\sum_{y=1}^{n} \tilde{\alpha}_{x y}^{I} \wp_{y}^{I R}(\varsigma-1) v_{13}-\sum_{y=1}^{n} \tilde{\alpha}_{x y}^{I} \wp_{y}^{I I}(\varsigma-1) v_{14} \\
& \left.-\sum_{y=1}^{n} \tilde{\alpha}_{x y}^{J} \wp_{y}^{I J}(\varsigma-1) v_{15}-\sum_{y=1}^{n} \tilde{\alpha}_{x y}^{J} \wp_{y}^{I K}(\varsigma-1) v_{16}-\sum_{y=1}^{n} \tilde{\alpha}_{y x}^{J} \wp_{x}^{I K} \frac{1}{v_{16}^{\zeta-1}}\right\} \frac{\left|d_{x}(t)\right|^{\varsigma}}{\varsigma} \\
& { }^{C} \mathcal{D}_{0, t}^{\sigma} \mathscr{V}\left(w_{x}(t)\right) \leq-\min _{1 \leq x \leq 1}\left\{\mathbf{a}_{x} \varsigma-\sum_{y=1}^{n} \tilde{\alpha}_{x y}^{R} \wp_{y}^{R R}(\varsigma-1) v_{1}-\sum_{y=1}^{n} \tilde{\alpha}_{y x}^{R} \wp_{x}^{R R} \frac{1}{v_{1}^{\varsigma-1}}-\sum_{y=1}^{n} \tilde{\alpha}_{x y}^{R} \wp_{y}^{R I}(\varsigma-1) v_{2}\right. \\
& -\sum_{y=1}^{n} \tilde{\alpha}_{x y}^{R} \wp_{y}^{R J}(\varsigma-1) v_{3}-\sum_{y=1}^{n} \tilde{\alpha}_{x y}^{R} \wp_{y}^{R K}(\varsigma-1) v_{4}+\sum_{y=1}^{n} \tilde{\alpha}_{x y}^{I} \wp_{y}^{I R}(\varsigma-1) v_{1}+\sum_{y=1}^{n} \tilde{\alpha}_{y x}^{I} \wp_{x}^{I R} \frac{1}{v_{1}^{\varsigma-1}} \\
& +\sum_{y=1}^{n} \tilde{\alpha}_{x y}^{I} \wp_{y}^{I I}(\varsigma-1) v_{2}+\sum_{y=1}^{n} \tilde{\alpha}_{x y}^{I} \wp_{y}^{I J}(\varsigma-1) v_{3}+\sum_{y=1}^{n} \tilde{\alpha}_{x y}^{I} \wp_{y}^{I K}(\varsigma-1) v_{4}+\sum_{y=1}^{n} \tilde{\alpha}_{x y}^{I} \wp_{y}^{J R}(\varsigma-1) v_{1}
\end{aligned}
$$


$+\sum_{y=1}^{n} \tilde{\alpha}_{y x}^{J} \wp_{x}^{J R} \frac{1}{v_{1}^{\varsigma-1}}+\sum_{y=1}^{n} \tilde{\alpha}_{x y}^{J} \wp_{y}^{J I}(\varsigma-1) v_{2}+\sum_{y=1}^{n} \tilde{\alpha}_{x y}^{J} \wp_{y}^{J J}(\varsigma-1) v_{3}+\sum_{y=1}^{n} \tilde{\alpha}_{x y}^{J} \wp_{y}^{J K}(\varsigma-1) v_{4}$

$+\sum_{y=1}^{n} \tilde{\alpha}_{x y}^{K} \wp_{y}^{K R}(\varsigma-1) v_{1}-\sum_{y=1}^{n} \tilde{\alpha}_{y x}^{K} \wp_{x}^{K R} \frac{1}{v_{1}^{\varsigma-1}}+\sum_{y=1}^{n} \tilde{\alpha}_{x y}^{K} \wp_{y}^{K I}(\varsigma-1) v_{2}+\sum_{y=1}^{n} \tilde{\alpha}_{x y}^{K} \wp_{y}^{K J}(\varsigma-1) v_{3}$

$+\sum_{y=1}^{n} \tilde{\alpha}_{x y}^{K} \wp_{y}^{K K}(\varsigma-1) v_{4}-\sum_{y=1}^{n} \tilde{\alpha}_{y x}^{R} \wp_{x}^{I R} \frac{1}{v_{5}^{S-1}}-\sum_{y=1}^{n} \tilde{\alpha}_{y x}^{I} \wp_{x}^{R R} \frac{1}{v_{5}^{S-1}}-\sum_{y=1}^{n} \tilde{\alpha}_{y x}^{I} \wp_{x}^{K R} \frac{1}{v_{5}^{S-1}}+\sum_{y=1}^{n} \tilde{\alpha}_{y x}^{K} \wp_{x}^{J R} \frac{1}{v_{5}^{S-1}}$

$-\sum_{y=1}^{n} \tilde{\alpha}_{y x}^{R} \wp_{x}^{J R} \frac{1}{v_{9}^{\varsigma-1}}-\sum_{y=1}^{n} \tilde{\alpha}_{y x}^{J} \wp_{x}^{R R} \frac{1}{v_{9}^{\varsigma-1}}-\sum_{y=1}^{n} \tilde{\alpha}_{y x}^{K} \wp_{x}^{I R} \frac{1}{v_{9}^{\varsigma-1}}+\sum_{y=1}^{n} \tilde{\alpha}_{y x}^{I} \wp_{x}^{K R} \frac{1}{v_{9}^{\varsigma-1}}-\sum_{y=1}^{n} \tilde{\alpha}_{y x}^{R} \wp_{x}^{K R} \frac{1}{v_{13}^{\varsigma-1}}$

$\left.-\sum_{y=1}^{n} \tilde{\alpha}_{y x}^{I} \wp_{x}^{J R} \frac{1}{v_{13}^{S-1}}-\sum_{y=1}^{n} \tilde{\alpha}_{y x}^{K} \wp_{x}^{R R} \frac{1}{v_{13}^{\varsigma-1}}+\sum_{y=1}^{n} \tilde{\alpha}_{y x}^{J} \wp_{x}^{I R} \frac{1}{v_{13}^{\varsigma-1}}\right\} \sum_{x=1}^{n} \frac{\left|a_{x}(t)\right|^{\varsigma}}{\varsigma}$

$-\min _{1 \leq x \leq 1}\left\{\mathbf{a}_{x} \varsigma-\sum_{y=1}^{n} \tilde{\alpha}_{y x}^{R} \wp_{x}^{R I} \frac{1}{v_{2}^{\varsigma-1}}+\sum_{y=1}^{n} \tilde{\alpha}_{y x}^{I} \wp_{x}^{I I} \frac{1}{v_{2}^{\varsigma-1}}+\sum_{y=1}^{n} \tilde{\alpha}_{y x}^{J} \wp_{x}^{J I} \frac{1}{v_{2}^{\varsigma-1}}+\sum_{y=1}^{n} \tilde{\alpha}_{y x}^{K} \wp_{x}^{K I} \frac{1}{v_{2}^{\varsigma-1}}\right.$

$-\sum_{y=1}^{n} \tilde{\alpha}_{x y}^{R} \wp_{y}^{I R}(\varsigma-1) v_{5}-\sum_{y=1}^{n} \tilde{\alpha}_{x y}^{R} \wp_{y}^{I I}(\varsigma-1) v_{6}-\sum_{y=1}^{n} \tilde{\alpha}_{y x}^{R} \wp_{x}^{I I} \frac{1}{v_{6}^{\varsigma-1}}-\sum_{y=1}^{n} \tilde{\alpha}_{x y}^{R} \wp_{y}^{I J}(\varsigma-1) v_{7}$

$-\sum_{y=1}^{n} \tilde{\alpha}_{x y}^{R} \wp_{y}^{I K}(\varsigma-1) v_{8}-\sum_{y=1}^{n} \tilde{\alpha}_{x y}^{I} \wp_{y}^{R R}(\varsigma-1) v_{5}-\sum_{y=1}^{n} \tilde{\alpha}_{x y}^{I} \wp_{y}^{R I}(\varsigma-1) v_{6}-\sum_{y=1}^{n} \tilde{\alpha}_{y x}^{I} \wp_{x}^{R I} \frac{1}{v_{6}^{\zeta-1}}$

$-\sum_{y=1}^{n} \tilde{\alpha}_{x y}^{I} \wp_{y}^{R J}(\varsigma-1) v_{7}-\sum_{y=1}^{n} \tilde{\alpha}_{x y}^{I} \wp_{y}^{R K}(\varsigma-1) v_{8}-\sum_{y=1}^{n} \tilde{\alpha}_{x y}^{I} \wp_{y}^{K R}(\varsigma-1) v_{5}-\sum_{y=1}^{n} \tilde{\alpha}_{x y}^{I} \wp_{y}^{K I}(\varsigma-1) v_{6}$

$-\sum_{y=1}^{n} \tilde{\alpha}_{y x}^{J} \wp_{x}^{K I} \frac{1}{v_{6}^{\varsigma-1}}-\sum_{y=1}^{n} \tilde{\alpha}_{x y}^{J} \wp_{y}^{K J}(\varsigma-1) v_{7}-\sum_{y=1}^{n} \tilde{\alpha}_{x y}^{J} \wp_{y}^{K K}(\varsigma-1) v_{8}+\sum_{y=1}^{n} \tilde{\alpha}_{x y}^{K} \wp_{y}^{J R}(\varsigma-1) v_{5}$

$+\sum_{y=1}^{n} \tilde{\alpha}_{x y}^{K} \wp_{y}^{J I}(\varsigma-1) v_{6}+\sum_{y=1}^{n} \tilde{\alpha}_{y x}^{K} \wp_{x}^{J I} \frac{1}{v_{6}^{\varsigma-1}}+\sum_{y=1}^{n} \tilde{\alpha}_{x y}^{K} \wp_{y}^{J J}(\varsigma-1) v_{7}+\sum_{y=1}^{n} \tilde{\alpha}_{x y}^{K} \wp_{y}^{J K}(\varsigma-1) v_{8}$

$-\sum_{y=1}^{n} \tilde{\alpha}_{y x}^{R} \wp_{x}^{J I} \frac{1}{v_{10}^{\varsigma-1}}-\sum_{y=1}^{n} \tilde{\alpha}_{y x}^{J} \wp_{x}^{R I} \frac{1}{v_{10}^{\varsigma-1}}-\sum_{y=1}^{n} \tilde{\alpha}_{y x}^{K} \wp_{x}^{I I} \frac{1}{v_{10}^{\varsigma-1}}+\sum_{y=1}^{n} \tilde{\alpha}_{y x}^{I} \wp_{x}^{K I} \frac{1}{v_{10}^{\varsigma-1}}$

$\left.-\sum_{y=1}^{n} \tilde{\alpha}_{y x}^{R} \wp_{x}^{K I} \frac{1}{v_{14}^{S-1}}-\sum_{y=1}^{n} \tilde{\alpha}_{y x}^{I} \wp_{x}^{I I} \frac{1}{v_{14}^{S-1}}-\sum_{y=1}^{n} \tilde{\alpha}_{y x}^{K} \wp_{x}^{R I} \frac{1}{v_{14}^{S-1}}+\sum_{y=1}^{n} \tilde{\alpha}_{y x}^{I} \wp_{x}^{I I} \frac{1}{v_{14}^{S-1}}\right\} \sum_{x=1}^{n} \frac{\left|b_{x}(t)\right|^{\varsigma}}{\varsigma}$

$-\min _{1 \leq x \leq 1}\left\{\mathbf{a}_{x} \varsigma-\sum_{y=1}^{n} \tilde{\alpha}_{y x}^{R} \wp_{x}^{R J} \frac{1}{v_{3}^{\varsigma-1}}+\sum_{y=1}^{n} \tilde{\alpha}_{y x}^{I} \wp_{x}^{I J} \frac{1}{v_{3}^{\varsigma-1}}+\sum_{y=1}^{n} \tilde{\alpha}_{y x}^{J} \wp_{x}^{J J} \frac{1}{v_{3}^{\varsigma-1}}+\sum_{y=1}^{n} \tilde{\alpha}_{y x}^{K} \wp_{x}^{K J} \frac{1}{v_{3}^{\varsigma-1}}\right.$

$-\sum_{y=1}^{n} \tilde{\alpha}_{y x}^{R} \wp_{x}^{I J} \frac{1}{v_{7}^{S-1}}-\sum_{y=1}^{n} \tilde{\alpha}_{y x}^{I} \wp_{x}^{R J} \frac{1}{v_{7}^{S-1}}-\sum_{y=1}^{n} \tilde{\alpha}_{y x}^{J} \wp_{x}^{K J} \frac{1}{v_{7}^{S-1}}+\sum_{y=1}^{n} \tilde{\alpha}_{y x}^{K} \wp_{x}^{J J} \frac{1}{v_{7}^{S-1}}-\sum_{y=1}^{n} \tilde{\alpha}_{x y}^{R} \wp_{y}^{J R}(\varsigma-1) v_{9}$

$-\sum_{y=1}^{n} \tilde{\alpha}_{x y}^{R} \wp_{y}^{J I}(\varsigma-1) v_{10}-\sum_{y=1}^{n} \tilde{\alpha}_{x y}^{R} \wp_{y}^{J J}(\varsigma-1) v_{11}-\sum_{y=1}^{n} \tilde{\alpha}_{y x}^{R} \wp_{x}^{J J} \frac{1}{v_{11}^{\varsigma-1}}-\sum_{y=1}^{n} \tilde{\alpha}_{x y}^{R} \wp_{y}^{J K}(\varsigma-1) v_{12}$

$-\sum_{y=1}^{n} \tilde{\alpha}_{x y}^{J} \wp_{y}^{R R}(\varsigma-1) v_{9}-\sum_{y=1}^{n} \tilde{\alpha}_{x y}^{J} \wp_{y}^{R I}(\varsigma-1) v_{10}-\sum_{y=1}^{n} \tilde{\alpha}_{x y}^{I} \wp_{y}^{R J}(\varsigma-1) v_{11}-\sum_{y=1}^{n} \tilde{\alpha}_{y x}^{J} \wp_{x}^{R J} \frac{1}{v_{11}^{S-1}}$

$-\sum_{y=1}^{n} \tilde{\alpha}_{x y}^{J} \wp_{y}^{R K}(\varsigma-1) v_{12}-\sum_{y=1}^{n} \tilde{\alpha}_{x y}^{K} \wp_{y}^{I R}(\varsigma-1) v_{9}-\sum_{y=1}^{n} \tilde{\alpha}_{x y}^{K} \wp_{y}^{I I}(\varsigma-1) v_{10}-\sum_{y=1}^{n} \tilde{\alpha}_{x y}^{K} \wp_{y}^{I J}(\varsigma-1) v_{11}$

$-\sum_{y=1}^{n} \tilde{\alpha}_{y x}^{K} \wp_{x}^{I I} \frac{1}{v_{11}^{\varsigma-1}}-\sum_{y=1}^{n} \tilde{\alpha}_{x y}^{K} \wp_{y}^{I K}(\varsigma-1) v_{12}+\sum_{y=1}^{n} \tilde{\alpha}_{x y}^{I} \wp_{y}^{K R}(\varsigma-1) v_{9}+\sum_{y=1}^{n} \tilde{\alpha}_{x y}^{I} \wp_{y}^{K I}(\varsigma-1) v_{10}$

$+\sum_{y=1}^{n} \tilde{\alpha}_{x y}^{I} \wp_{y}^{K J}(\varsigma-1) v_{11}+\sum_{y=1}^{n} \tilde{\alpha}_{y x}^{I} \wp_{x}^{K J} \frac{1}{v_{11}^{S-1}}+\sum_{y=1}^{n} \tilde{\alpha}_{x y}^{I} \wp_{y}^{K K}(\varsigma-1) v_{12}-\sum_{y=1}^{n} \tilde{\alpha}_{y x}^{R} \wp_{x}^{K J} \frac{1}{v_{15}^{\varsigma-1}}$

$\left.-\sum_{y=1}^{n} \tilde{\alpha}_{y x}^{I} \wp_{x}^{J J} \frac{1}{v_{15}^{\varsigma-1}}-\sum_{y=1}^{n} \tilde{\alpha}_{y x}^{K} \wp_{x}^{R J} \frac{1}{v_{15}^{\varsigma-1}}+\sum_{y=1}^{n} \tilde{\alpha}_{y x}^{J} \wp_{x}^{I J} \frac{1}{v_{15}^{S-1}}\right\} \sum_{x=1}^{n} \frac{\left|c_{x}(t)\right|^{\varsigma}}{\varsigma}$ 


$$
\begin{aligned}
& -\min _{1 \leq x \leq 1}\left\{\mathbf{a}_{x} \varsigma-\sum_{y=1}^{n} \tilde{\alpha}_{y x}^{R} \wp_{x}^{R K} \frac{1}{v_{4}^{\varsigma-1}}+\sum_{y=1}^{n} \tilde{\alpha}_{y x}^{I} \wp_{x}^{I K} \frac{1}{v_{4}^{\varsigma-1}}+\sum_{y=1}^{n} \tilde{\alpha}_{y x}^{J} \wp_{x}^{J K} \frac{1}{v_{4}^{\varsigma-1}}+\sum_{y=1}^{n} \tilde{\alpha}_{y x}^{K} \wp_{x}^{K K} \frac{1}{v_{4}^{\varsigma-1}}\right. \\
& -\sum_{y=1}^{n} \tilde{\alpha}_{y x}^{R} \wp_{x}^{I K} \frac{1}{v_{8}^{\varsigma-1}}-\sum_{y=1}^{n} \tilde{\alpha}_{y x}^{I} \wp_{x}^{R K} \frac{1}{v_{8}^{\varsigma-1}}-\sum_{y=1}^{n} \tilde{\alpha}_{y x}^{J} \wp_{x}^{K K} \frac{1}{v_{8}^{\varsigma-1}}+\sum_{y=1}^{n} \tilde{\alpha}_{y x}^{K} \wp_{x}^{J K} \frac{1}{v_{8}^{\varsigma-1}}-\sum_{y=1}^{n} \tilde{\alpha}_{y x}^{R} \wp_{x}^{J K} \frac{1}{v_{12}^{\varsigma-1}} \\
& -\sum_{y=1}^{n} \tilde{\alpha}_{y x}^{J} \wp_{x}^{R K} \frac{1}{v_{12}^{\varsigma-1}}-\sum_{y=1}^{n} \tilde{\alpha}_{y x}^{K} \wp_{x}^{I K} \frac{1}{v_{12}^{\varsigma-1}}+\sum_{y=1}^{n} \tilde{\alpha}_{y x}^{I} \wp_{x}^{K K} \frac{1}{v_{12}^{\varsigma-1}}-\sum_{y=1}^{n} \tilde{\alpha}_{x y}^{R} \wp_{y}^{K R}(\varsigma-1) v_{13} \\
& -\sum_{y=1}^{n} \tilde{\alpha}_{x y}^{R} \wp_{y}^{K I}(\varsigma-1) v_{14}-\sum_{y=1}^{n} \tilde{\alpha}_{x y}^{R} \wp_{y}^{K J}(\varsigma-1) v_{15}-\sum_{y=1}^{n} \tilde{\alpha}_{x y}^{R} \wp_{y}^{K K}(\varsigma-1) v_{16}-\sum_{y=1}^{n} \tilde{\alpha}_{y x}^{R} \wp_{x}^{K K} \frac{1}{v_{16}^{\varsigma-1}} \\
& -\sum_{y=1}^{n} \tilde{\alpha}_{x y}^{I} \wp_{y}^{J R}(\varsigma-1) v_{13}-\sum_{y=1}^{n} \tilde{\alpha}_{x y}^{I} \wp_{y}^{J I}(\varsigma-1) v_{14}-\sum_{y=1}^{n} \tilde{\alpha}_{x y}^{I} \wp_{y}^{J J}(\varsigma-1) v_{15}-\sum_{y=1}^{n} \tilde{\alpha}_{x y}^{I} \wp_{y}^{J K}(\varsigma-1) v_{16} \\
& -\sum_{y=1}^{n} \tilde{\alpha}_{y x}^{I} \wp_{x}^{J K} \frac{1}{v_{16}^{\varsigma-1}}-\sum_{y=1}^{n} \tilde{\alpha}_{x y}^{K} \wp_{y}^{R R}(\varsigma-1) v_{13}-\sum_{y=1}^{n} \tilde{\alpha}_{x y}^{K} \wp_{y}^{R I}(\varsigma-1) v_{14}-\sum_{y=1}^{n} \tilde{\alpha}_{x y}^{K} \wp_{y}^{R J}(\varsigma-1) v_{15} \\
& -\sum_{y=1}^{n} \tilde{\alpha}_{x y}^{K} \wp_{y}^{R K}(\varsigma-1) v_{16}-\sum_{y=1}^{n} \tilde{\alpha}_{y x}^{K} \wp_{x}^{R K} \frac{1}{v_{16}^{\varsigma-1}}+\sum_{y=1}^{n} \tilde{\alpha}_{x y}^{J} \wp_{y}^{I R}(\varsigma-1) v_{13}+\sum_{y=1}^{n} \tilde{\alpha}_{x y}^{J} \wp_{y}^{I I}(\varsigma-1) v_{14} \\
& \left.+\sum_{y=1}^{n} \tilde{\alpha}_{x y}^{J} \wp_{y}^{I J}(\varsigma-1) v_{15}+\sum_{y=1}^{n} \tilde{\alpha}_{x y}^{J} \wp_{y}^{I K}(\varsigma-1) v_{16}+\sum_{y=1}^{n} \tilde{\alpha}_{y x}^{J} \wp_{x}^{I K} \frac{1}{v_{16}^{\varsigma-1}}\right\} \sum_{x=1}^{n} \frac{\left|d_{x}(t)\right|^{\varsigma}}{\varsigma}, \\
& :=-\omega_{1} \sum_{x=1}^{n} \frac{\left|a_{x}(t)\right|^{\varsigma}}{\varsigma}-\omega_{2} \sum_{x=1}^{n} \frac{\left|b_{x}(t)\right|^{\varsigma}}{\varsigma}-\omega_{3} \sum_{x=1}^{n} \frac{\left|c_{x}(t)\right|^{\varsigma}}{\varsigma}-\omega_{4} \sum_{x=1}^{n} \frac{\left|d_{x}(t)\right|^{\varsigma}}{\varsigma},
\end{aligned}
$$

where

$$
\begin{aligned}
\omega_{1}= & \min _{1 \leq x \leq 1}\left\{\mathbf{a}_{x} \varsigma-\sum_{y=1}^{n} \tilde{\alpha}_{x y}^{R} \wp_{y}^{R R}(\varsigma-1) v_{1}-\sum_{y=1}^{n} \tilde{\alpha}_{y x}^{R} \wp_{x}^{R R} \frac{1}{v_{1}^{\varsigma-1}}-\sum_{y=1}^{n} \tilde{\alpha}_{x y}^{R} \wp_{y}^{R I}(\varsigma-1) v_{2}\right. \\
& -\sum_{y=1}^{n} \tilde{\alpha}_{x y}^{R} \wp_{y}^{R J}(\varsigma-1) v_{3}-\sum_{y=1}^{n} \tilde{\alpha}_{x y}^{R} \wp_{y}^{R K}(\varsigma-1) v_{4}+\sum_{y=1}^{n} \tilde{\alpha}_{x y}^{I} \wp_{y}^{I R}(\varsigma-1) v_{1}+\sum_{y=1}^{n} \tilde{\alpha}_{y x}^{I} \wp_{x}^{I R} \frac{1}{v_{1}^{\varsigma-1}} \\
& +\sum_{y=1}^{n} \tilde{\alpha}_{x y}^{I} \wp_{y}^{I I}(\varsigma-1) v_{2}+\sum_{y=1}^{n} \tilde{\alpha}_{x y}^{I} \wp_{y}^{I J}(\varsigma-1) v_{3}+\sum_{y=1}^{n} \tilde{\alpha}_{x y}^{I} \wp_{y}^{I K}(\varsigma-1) v_{4}+\sum_{y=1}^{n} \tilde{\alpha}_{x y}^{J} \wp_{y}^{J R}(\varsigma-1) v_{1} \\
& +\sum_{y=1}^{n} \tilde{\alpha}_{y x}^{J} \wp_{x}^{J R} \frac{1}{v_{1}^{\varsigma-1}}+\sum_{y=1}^{n} \tilde{\alpha}_{x y}^{J} \wp_{y}^{J I}(\varsigma-1) v_{2}+\sum_{y=1}^{n} \tilde{\alpha}_{x y}^{J} \wp_{y}^{J J}(\varsigma-1) v_{3}+\sum_{y=1}^{n} \tilde{\alpha}_{x y}^{J} \wp_{y}^{J K}(\varsigma-1) v_{4} \\
& +\sum_{y=1}^{n} \tilde{\alpha}_{x y}^{K} \wp_{y}^{K R}(\varsigma-1) v_{1}+\sum_{y=1}^{n} \tilde{\alpha}_{x y}^{K} \wp_{y}^{K R}(\varsigma-1) v_{1}+\sum_{y=1}^{n} \tilde{\alpha}_{x y}^{K} \wp_{y}^{K I}(\varsigma-1) v_{2}+\sum_{y=1}^{n} \tilde{\alpha}_{x y}^{K} \wp_{y}^{K J}(\varsigma-1) v_{3} \\
& +\sum_{y=1}^{n} \tilde{\alpha}_{x y}^{K} \wp_{y}^{K K}(\varsigma-1) v_{4}-\sum_{y=1}^{n} \tilde{\alpha}_{y x}^{R} \wp_{x}^{I R} \frac{1}{v_{5}^{\varsigma-1}}-\sum_{y=1}^{n} \tilde{\alpha}_{y x}^{I} \wp_{x}^{R R} \frac{1}{v_{5}^{\varsigma-1}}-\sum_{y=1}^{n} \tilde{\alpha}_{y x}^{J} \wp_{x}^{K R} \frac{1}{v_{5}^{\varsigma-1}}+\sum_{y=1}^{n} \tilde{\alpha}_{y x}^{K} \wp_{x}^{J R} \frac{1}{v_{5}^{\varsigma-1}} \\
& -\sum_{y=1}^{n} \tilde{\alpha}_{y x}^{R} \wp_{x}^{J R} \frac{1}{v_{9}^{\varsigma-1}}-\sum_{y=1}^{n} \tilde{\alpha}_{y x}^{J} \wp_{x}^{R R} \frac{1}{v_{9}^{\varsigma-1}}-\sum_{y=1}^{n} \tilde{\alpha}_{y x}^{K} \wp_{x}^{I R} \frac{1}{v_{9}^{\varsigma-1}}+\sum_{y=1}^{n} \tilde{\alpha}_{y x}^{I} \wp_{x}^{K R} \frac{1}{v_{9}^{\varsigma-1}}-\sum_{y=1}^{n} \tilde{\alpha}_{y x}^{R} \wp_{x}^{K R} \frac{1}{v_{13}^{\varsigma-1}} \\
& \left.-\sum_{y=1}^{n} \tilde{\alpha}_{y x}^{I} \wp_{x}^{J R} \frac{1}{v_{13}^{\varsigma-1}}-\sum_{y=1}^{n} \tilde{\alpha}_{y x}^{K} \wp_{x}^{R R} \frac{1}{v_{13}^{\varsigma-1}}+\sum_{y=1}^{n} \tilde{\alpha}_{y x}^{J} \wp_{x}^{I R} \frac{1}{v_{13}^{\varsigma-1}}\right\},
\end{aligned}
$$




$$
\begin{aligned}
& \omega_{2}=\min _{1 \leq x \leq 1}\left\{\mathbf{a}_{x} \varsigma-\sum_{y=1}^{n} \tilde{\alpha}_{y x}^{R} \wp_{x}^{R I} \frac{1}{v_{2}^{\varsigma-1}}+\sum_{y=1}^{n} \tilde{\alpha}_{y x}^{I} \wp_{x}^{I I} \frac{1}{v_{2}^{\varsigma-1}}+\sum_{y=1}^{n} \tilde{\alpha}_{y x}^{J} \wp_{x}^{J I} \frac{1}{v_{2}^{\varsigma-1}}+\sum_{y=1}^{n} \tilde{\alpha}_{y x}^{K} \wp_{x}^{K I} \frac{1}{v_{2}^{\varsigma-1}}\right. \\
& -\sum_{y=1}^{n} \tilde{\alpha}_{x y}^{R} \wp_{y}^{I R}(\varsigma-1) v_{5}-\sum_{y=1}^{n} \tilde{\alpha}_{x y}^{R} \wp_{y}^{I I}(\varsigma-1) v_{6}-\sum_{y=1}^{n} \tilde{\alpha}_{y x}^{R} \wp_{x}^{I I} \frac{1}{v_{6}^{\zeta-1}}-\sum_{y=1}^{n} \tilde{\alpha}_{x y}^{R} \wp_{y}^{I J}(\varsigma-1) v_{7} \\
& -\sum_{y=1}^{n} \tilde{\alpha}_{x y}^{R} \wp_{y}^{I K}(\varsigma-1) v_{8}-\sum_{y=1}^{n} \tilde{\alpha}_{x y}^{I} \wp_{y}^{R R}(\varsigma-1) v_{5}-\sum_{y=1}^{n} \tilde{\alpha}_{x y}^{I} \wp_{y}^{R I}(\varsigma-1) v_{6}-\sum_{y=1}^{n} \tilde{\alpha}_{y x}^{I} \wp_{x}^{R I} \frac{1}{v_{6}^{\varsigma-1}} \\
& -\sum_{y=1}^{n} \tilde{\alpha}_{x y}^{I} \wp_{y}^{R J}(\varsigma-1) v_{7}-\sum_{y=1}^{n} \tilde{\alpha}_{x y}^{I} \wp_{y}^{R K}(\varsigma-1) v_{8}-\sum_{y=1}^{n} \tilde{\alpha}_{x y}^{I} \wp_{y}^{K R}(\varsigma-1) v_{5}-\sum_{y=1}^{n} \tilde{\alpha}_{x y}^{I} \wp_{y}^{R I}(\varsigma-1) v_{6} \\
& -\sum_{y=1}^{n} \tilde{\alpha}_{y x}^{J} \wp_{x}^{K I} \frac{1}{v_{6}^{\varsigma-1}}-\sum_{y=1}^{n} \tilde{\alpha}_{x y}^{J} \wp_{y}^{K J}(\varsigma-1) v_{7}-\sum_{y=1}^{n} \tilde{\alpha}_{x y}^{J} \wp_{y}^{K K}(\varsigma-1) v_{8}+\sum_{y=1}^{n} \tilde{\alpha}_{x y}^{K} \wp_{y}^{J R}(\varsigma-1) v_{5} \\
& +\sum_{y=1}^{n} \tilde{\alpha}_{x y}^{K} \wp_{y}^{J I}(\varsigma-1) v_{6}+\sum_{y=1}^{n} \tilde{\alpha}_{y x}^{K} \wp_{x}^{J I} \frac{1}{v_{6}^{\varsigma-1}}+\sum_{y=1}^{n} \tilde{\alpha}_{x y}^{K} \wp_{y}^{J J}(\varsigma-1) v_{7}+\sum_{y=1}^{n} \tilde{\alpha}_{x y}^{K} \wp_{y}^{J K}(\varsigma-1) v_{8} \\
& -\sum_{y=1}^{n} \tilde{\alpha}_{y x}^{R} \wp_{x}^{J I} \frac{1}{v_{10}^{\varsigma-1}}-\sum_{y=1}^{n} \tilde{\alpha}_{y x}^{J} \wp_{x}^{R I} \frac{1}{v_{10}^{\varsigma-1}}-\sum_{y=1}^{n} \tilde{\alpha}_{y x}^{K} \wp_{x}^{I I} \frac{1}{v_{10}^{S-1}}+\sum_{y=1}^{n} \tilde{\alpha}_{y x}^{I} \wp_{x}^{K I} \frac{1}{v_{10}^{S-1}} \\
& \left.-\sum_{y=1}^{n} \tilde{\alpha}_{y x}^{R} \wp_{x}^{K I} \frac{1}{v_{14}^{\varsigma-1}}-\sum_{y=1}^{n} \tilde{\alpha}_{y x}^{I} \wp_{x}^{J I} \frac{1}{v_{14}^{\varsigma-1}}-\sum_{y=1}^{n} \tilde{\alpha}_{y x}^{K} \wp_{x}^{R I} \frac{1}{v_{14}^{S-1}}-\sum_{y=1}^{n} \tilde{\alpha}_{y x}^{I} \wp_{x}^{I I} \frac{1}{v_{14}^{\varsigma-1}}\right\}, \\
& \omega_{3}=\min _{1 \leq x \leq 1}\left\{\mathbf{a}_{x} \varsigma-\sum_{y=1}^{n} \tilde{\alpha}_{y x}^{R} \wp_{x}^{R J} \frac{1}{v_{3}^{\zeta-1}}+\sum_{y=1}^{n} \tilde{\alpha}_{y x}^{I} \wp_{x}^{I J} \frac{1}{v_{3}^{\varsigma-1}}+\sum_{y=1}^{n} \tilde{\alpha}_{y x}^{J} \wp_{x}^{J J} \frac{1}{v_{3}^{\zeta-1}}+\sum_{y=1}^{n} \tilde{\alpha}_{y x}^{K} \wp_{x}^{K J} \frac{1}{v_{3}^{\zeta-1}}\right. \\
& -\sum_{y=1}^{n} \tilde{\alpha}_{y x}^{R} \wp_{x}^{I J} \frac{1}{v_{7}^{\varsigma-1}}-\sum_{y=1}^{n} \tilde{\alpha}_{y x}^{I} \wp_{x}^{R J} \frac{1}{v_{7}^{\varsigma-1}}-\sum_{y=1}^{n} \tilde{\alpha}_{y x}^{J} \wp_{x}^{K J} \frac{1}{v_{7}^{\varsigma-1}}+\sum_{y=1}^{n} \tilde{\alpha}_{y x}^{K} \wp_{x}^{J J} \frac{1}{v_{7}^{\varsigma-1}}-\sum_{y=1}^{n} \tilde{\alpha}_{x y}^{R} \wp_{y}^{J R}(\varsigma-1) v_{9} \\
& -\sum_{y=1}^{n} \tilde{\alpha}_{x y}^{R} \wp_{y}^{J I}(\varsigma-1) v_{10}-\sum_{y=1}^{n} \tilde{\alpha}_{x y}^{R} \wp_{y}^{J J}(\varsigma-1) v_{11}-\sum_{y=1}^{n} \tilde{\alpha}_{y x}^{R} \wp_{x}^{J J} \frac{1}{v_{11}^{\varsigma-1}}-\sum_{y=1}^{n} \tilde{\alpha}_{x y}^{R} \wp_{y}^{J K}(\varsigma-1) v_{12} \\
& -\sum_{y=1}^{n} \tilde{\alpha}_{x y}^{J} \wp_{y}^{R R}(\varsigma-1) v_{9}-\sum_{y=1}^{n} \tilde{\alpha}_{x y}^{J} \wp_{y}^{R I}(\varsigma-1) v_{10}-\sum_{y=1}^{n} \tilde{\alpha}_{x y}^{J} \wp_{y}^{R J}(\varsigma-1) v_{11}-\sum_{y=1}^{n} \tilde{\alpha}_{y x}^{J} \wp_{x}^{R J} \frac{1}{v_{11}^{\varsigma-1}} \\
& -\sum_{y=1}^{n} \tilde{\alpha}_{x y}^{J} \wp_{y}^{R K}(\varsigma-1) v_{12}-\sum_{y=1}^{n} \tilde{\alpha}_{x y}^{K} \wp_{y}^{I R}(\varsigma-1) v_{9}-\sum_{y=1}^{n} \tilde{\alpha}_{x y}^{K} \wp_{y}^{I I}(\varsigma-1) v_{10}-\sum_{y=1}^{n} \tilde{\alpha}_{x y}^{K} \wp_{y}^{I J}(\varsigma-1) v_{11} \\
& -\sum_{y=1}^{n} \tilde{\alpha}_{y x}^{K} \wp_{x}^{I J} \frac{1}{v_{11}^{\varsigma-1}}-\sum_{y=1}^{n} \tilde{\alpha}_{x y}^{K} \wp_{y}^{I K}(\varsigma-1) v_{12}+\sum_{y=1}^{n} \tilde{\alpha}_{x y}^{I} \wp_{y}^{K R}(\varsigma-1) v_{9}+\sum_{y=1}^{n} \tilde{\alpha}_{x y}^{I} \wp_{y}^{K I}(\varsigma-1) v_{10} \\
& +\sum_{y=1}^{n} \tilde{\alpha}_{x y}^{I} \wp_{y}^{K J}(\varsigma-1) v_{11}+\sum_{y=1}^{n} \tilde{\alpha}_{y x}^{I} \wp_{x}^{K J} \frac{1}{v_{11}^{\varsigma-1}}+\sum_{y=1}^{n} \tilde{\alpha}_{x y}^{I} \wp_{y}^{K K}(\varsigma-1) v_{12}-\sum_{y=1}^{n} \tilde{\alpha}_{y x}^{R} \wp_{x}^{K J} \frac{1}{v_{15}^{\varsigma-1}} \\
& \left.-\sum_{y=1}^{n} \tilde{\alpha}_{y x}^{I} \wp_{x}^{J J} \frac{1}{v_{15}^{\varsigma-1}}-\sum_{y=1}^{n} \tilde{\alpha}_{y x}^{K} \wp_{x}^{R J} \frac{1}{v_{15}^{\varsigma-1}}+\sum_{y=1}^{n} \tilde{\alpha}_{y x}^{J} \wp_{x}^{I J} \frac{1}{v_{15}^{\varsigma-1}}\right\} \\
& \boldsymbol{\omega}_{4}=\min _{1 \leq x \leq 1}\left\{\mathbf{a}_{x} \varsigma-\sum_{y=1}^{n} \tilde{\alpha}_{y x}^{R} \wp_{x}^{R K} \frac{1}{v_{4}^{\varsigma-1}}+\sum_{y=1}^{n} \tilde{\alpha}_{y x}^{I} \wp_{x}^{I K} \frac{1}{v_{4}^{\varsigma-1}}+\sum_{y=1}^{n} \tilde{\alpha}_{y x}^{J} \wp_{x}^{J K} \frac{1}{v_{4}^{\varsigma-1}}+\sum_{y=1}^{n} \tilde{\alpha}_{y x}^{K} \wp_{x}^{K K} \frac{1}{v_{4}^{\varsigma-1}}\right. \\
& -\sum_{y=1}^{n} \tilde{\alpha}_{y x}^{R} \wp_{x}^{I K} \frac{1}{v_{8}^{\varsigma-1}}-\sum_{y=1}^{n} \tilde{\alpha}_{y x}^{I} \wp_{x}^{R K} \frac{1}{v_{8}^{\varsigma-1}}-\sum_{y=1}^{n} \tilde{\alpha}_{y x}^{J} \wp_{x}^{K K} \frac{1}{v_{8}^{\varsigma-1}}+\sum_{y=1}^{n} \tilde{\alpha}_{y x}^{K} \wp_{x}^{J K} \frac{1}{v_{8}^{\varsigma-1}}-\sum_{y=1}^{n} \tilde{\alpha}_{y x}^{R} \wp_{x}^{J K} \frac{1}{v_{12}^{\varsigma-1}} \\
& -\sum_{y=1}^{n} \tilde{\alpha}_{y x x}^{J} \wp_{x}^{R K} \frac{1}{v_{12}^{\varsigma-1}}-\sum_{y=1}^{n} \tilde{\alpha}_{y x}^{K} \wp_{x}^{I K} \frac{1}{v_{12}^{\varsigma-1}}+\sum_{y=1}^{n} \tilde{\alpha}_{y x}^{I} \wp_{x}^{K K} \frac{1}{v_{12}^{\varsigma-1}}-\sum_{y=1}^{n} \tilde{\alpha}_{x y}^{R} \wp_{y}^{K R}(\varsigma-1) v_{13} \\
& -\sum_{y=1}^{n} \tilde{\alpha}_{x y}^{R} \wp_{y}^{K I}(\varsigma-1) v_{14}-\sum_{y=1}^{n} \tilde{\alpha}_{x y}^{R} \wp_{y}^{K J}(\varsigma-1) v_{15}-\sum_{y=1}^{n} \tilde{\alpha}_{x y}^{R} \wp_{y}^{K K}(\varsigma-1) v_{16}-\sum_{y=1}^{n} \tilde{\alpha}_{y x}^{R} \wp_{x}^{K K} \frac{1}{v_{16}^{\varsigma-1}} \\
& -\sum_{y=1}^{n} \tilde{\alpha}_{x y}^{I} \wp_{y}^{J R}(\varsigma-1) v_{13}-\sum_{y=1}^{n} \tilde{\alpha}_{x y}^{I} \wp_{y}^{J I}(\varsigma-1) v_{14}-\sum_{y=1}^{n} \tilde{\alpha}_{x y}^{I} \wp_{y}^{J J}(\varsigma-1) v_{15}-\sum_{y=1}^{n} \tilde{\alpha}_{x y}^{I} \wp_{y}^{J K}(\varsigma-1) v_{16} \\
& -\sum_{y=1}^{n} \tilde{\alpha}_{y x}^{I} \wp_{x}^{J K} \frac{1}{v_{16}^{\varsigma-1}}-\sum_{y=1}^{n} \tilde{\alpha}_{x y}^{K} \wp_{y}^{R R}(\varsigma-1) v_{13}-\sum_{y=1}^{n} \tilde{\alpha}_{x y}^{K} \wp_{y}^{R I}(\varsigma-1) v_{14}-\sum_{y=1}^{n} \tilde{\alpha}_{x y}^{K} \wp_{y}^{R J}(\varsigma-1) v_{15} \\
& -\sum_{y=1}^{n} \tilde{\alpha}_{x y}^{K} \wp_{y}^{R K}(\varsigma-1) v_{16}-\sum_{y=1}^{n} \tilde{\alpha}_{y x}^{K} \wp_{x}^{R K} \frac{1}{v_{16}^{S-1}}+\sum_{y=1}^{n} \tilde{\alpha}_{x y}^{I} \wp_{y}^{I R}(\varsigma-1) v_{13}+\sum_{y=1}^{n} \tilde{\alpha}_{x y}^{I} \wp_{y}^{I I}(\varsigma-1) v_{14} \\
& \left.+\sum_{y=1}^{n} \tilde{\alpha}_{x y}^{J} \wp_{y}^{I J}(\varsigma-1) v_{15}+\sum_{y=1}^{n} \tilde{\alpha}_{x y}^{I} \wp_{y}^{I K}(\varsigma-1) v_{16}+\sum_{y=1}^{n} \tilde{\alpha}_{y x}^{I} \wp_{x}^{I K} \frac{1}{v_{16}^{\varsigma-1}}\right\} .
\end{aligned}
$$

From (9)-(12), we can select a constant $\lambda>0$, such that $\min \left(\omega_{1}, \omega_{2}, \omega_{3}, \omega_{4}\right) \geq \lambda>0$. 
That is, we can conclude that

$$
{ }^{C} \mathcal{D}_{0, t}^{\sigma} \mathscr{V}\left(w_{x}(t)\right) \leq-\lambda \mathscr{V}\left(w_{x}(t)\right), t \geq 0 .
$$

Thus, there exists a nonnegative function $\mathscr{F}(t)$ satisfying

$$
{ }^{C} \mathcal{D}_{0, t}^{\sigma} \mathscr{V}\left(w_{x}(t)\right)+\mathscr{F}(t)=-\lambda \mathscr{V}\left(w_{x}(t)\right), t \geq 0 .
$$

Taking the Laplace transform on both sides of (A25), it follows that

$$
\mathscr{V}(\tau)=\frac{\tau^{\sigma-1}}{\tau^{\sigma}+\lambda} \mathscr{V}(0)-\frac{\mathscr{F}(\tau)}{\tau^{\sigma}+\lambda}
$$

where $\mathscr{V}(\tau)=\mathcal{L}\{\mathscr{V}(t)\}, \mathscr{F}(\tau)=\mathcal{L}\{\mathscr{F}(t)\}$

By the inverse Laplace transform, we obtain

$$
\mathscr{V}(t)=\mathscr{V}(0) \mathscr{E}_{\sigma}\left(-\lambda t^{\sigma}\right)-\mathscr{F}(t) * t^{\sigma-1} \mathscr{E}_{\sigma, \sigma}\left(-\lambda t^{\sigma}\right), t \geq 0,
$$

where $*$ denotes the convolution operator. From Lemma 2, $\mathscr{F}(t), t^{\sigma-1} \mathscr{E}_{\sigma, \sigma}\left(-\lambda t^{\sigma}\right)$ are all nonnegative functions.

Then, we can obtain

$$
\mathscr{V}(t) \leq \mathscr{V}(0) \mathscr{E}_{\sigma}\left(-\lambda t^{\sigma}\right), t \geq 0 .
$$

By Definitions (5) and (6), the error system (7) is globally Mittag-Leffler stable. This completes the proof.

\section{References}

1. Chua, L. Memristor: The missing circuit element. IEEE Trans. Circuits Theory 1971, 18, 507-519. [CrossRef]

2. Strukov, D.; Snider, G.; Stewart, D.; Williams, R. The missing memristor found. Nature 2008, 453, 80-83. [CrossRef] [PubMed]

3. Chua, L. Resistance switching memories are memristors. Appl. Phys. A 2011, 102, 765-783. [CrossRef]

4. Duan, S.; Hu, X.; Dong, Z.; Wang, L.; Mazumder, P. Memristor-based cellular nonlinear/neural network: design, analysis and applications. IEEE Trans. Neural Netw. Learn. Syst. 2015, 26, 1202-1213. [CrossRef] [PubMed]

5. Aubin, J.; Cellina, A. Differential Inclusions: Set-Valued Maps and Viability Theory; Springer: Berlin, Germany, 1984.

6. Jo, S.; Chang, T.; Ebong, I.; Bhadviya, B.; Mazumder, P.; Lu, W. Nanoscale memristor device as synapse in neuromorphic systems. Nano Lett. 2010, 10, 1297-1301. [CrossRef] [PubMed]

7. Sah, M.; Yang, C.; Kim, H.; Chua, L. A voltage mode memristor bridge synaptic circuit with memristor emulators. Sensors 2012, 12, 3587-3604. [CrossRef]

8. Adhikari, S.; Yang, C.; Kim, H.; Chua, L. Memristor bridge synapse-based neural network and its learning. IEEE Trans. Neural Netw. Learn. Syst. 2012, 23, 1426-1435. [CrossRef]

9. Chen, J.; Zeng, Z.; Jiang, P. Global Mittag-Leffler stability and synchronization of memristor-based fractional-order neural networks. Neural Netw. 2014, 51, 1-8. [CrossRef]

10. Chen, L.; Wu, R.; Cao, J.; Liu, J. Stability and synchronization of memristor-based fractional-order delayed neural networks. Neural Netw. 2015, 71, 37-44. [CrossRef]

11. Wu, A.; Zeng, Z. Global Mittag-Lefler stabilization of fractional-order memristive neural networks. IEEE Trans. Neural New. Learn. Syst. 2017, 28, 206-217. [CrossRef]

12. Liu, D.; Zhu, S.; Chang, W. Global exponential stability of stochastic memristor-based complex-valued neural networks with time delays. Nonlinear Dyn. 2017, 90, 915-934. [CrossRef]

13. Tu, Z.; Cao, J.; Alsaedi, A.; Alsaadi, F.E.; Hayat, T. Global Lagrange stability of complex-valued neural networks of neutral type with time-varying delays. Complexity 2016, 21, 438-450. [CrossRef] 
14. Zhang, Z.; Liu, X.; Chen, J.; Guo, R.; Zhou, S. Further stability analysis for delayed complex-valued recurrent neural networks. Neurocomputing 2017, 251, 81-89. [CrossRef]

15. Kwon, O.M.; Park, J.H. New delay-dependent robust stability criterion for uncertain neural networks with time-varying delays. Appl. Math. Comput. 2008, 205, 417-427. [CrossRef]

16. Guo, J.; Meng, Z.; Xiang, Z. Passivity analysis of stochastic memristor-based complex-valued recurrent neural networks with mixed time-varying delays. Neural Process. Lett. 2018, 47, 1097-1113. [CrossRef]

17. Nitta, T. Solving the XOR problem and the detection of symmetry using a single complex-valued neuron. Neural Netw. 2003, 16, 1101-1105. [CrossRef]

18. Goh, S.L.; Chen, M.; Popovic, D.H.; Aihara, K.; Obradovic, D.; Mandic, D.P. Complex-valued forecasting of wind profile. Renew. Energ. 2006, 31, 1733-1750. [CrossRef]

19. Samidurai, R.; Sriraman, R.; Cao, J.; Tu, Z. Effects of leakage delay on global asymptotic stability of complex-valued neural networks with interval time-varying delays via new complex-valued Jensen's inequality. Int. J. Adapt. Control and Signal Process. 2018, 32, 1294-1312. [CrossRef]

20. Guo, R.; Zhang, Z.; Liu, X.; Lin, C. Existence, uniqueness, and exponential stability analysis for complex-valued memristor-based BAM neural networks with time delays. Appl. Math. Comput. 2017, 311, 100-117. [CrossRef]

21. Zhang, Z.; Liu, X.; Guo, R.; Lin, C. Finite-time stability for delayed complex-valued BAM neural networks. Neural Process. Lett. 2018, 48, 179-193. [CrossRef]

22. Zhang, F. Quaternions and matrices of quaternions. Linear Algebra Appl. 1997, 251, 21-57. [CrossRef]

23. Arena, P.; Fortuna, L.; Muscato, G.; Xibilia, M.G. Multilayer perceptrons to approximate quaternion-valued functions. Neural Netw. 1997, 10, 335-342. [CrossRef]

24. Isokawa, T.; Nishimura, H.; Kamiura, N.; Matsui, N. Associative memory in quaternionic Hopfield neural network. Int. J. Neural Syst. 2008, 18, 135-145. [CrossRef] [PubMed]

25. Minemoto, T.; Isokawa, T.; Nishimura, H.; Matsui, N. Quaternionic multistate Hopfield neural network with extended projection rule. Artif. Life Robot. 2016, 21, 106-111. [CrossRef]

26. Liu, Y.; Zhang, D.; Lu, J. Global exponential stability for quaternion-valued recurrent neural networks with time-varying delays. Nonlinear Dyn. 2017, 87, 553-565. [CrossRef]

27. Li, H.L.; Jiang, H.; Cao, J. Global synchronization of fractional-order quaternion-valued neural networks with leakage and discrete delays. Neurocomputing 2019. [CrossRef]

28. Tu, Z.; Zhao, Y.; Ding, N.; Feng, Y.; Zhang, W. Stability analysis of quaternion-valued neural networks with both discrete and distributed delays. Appl. Math. Comput. 2019, 343, 342-353. [CrossRef]

29. You, X.; Song, Q.; Liang, J.; Liu, Y.G.; Alsaadi, F.E. Global $\mu$-stability of quaternion-valued neural networks with mixed time-varying delays. Neurocomputing 2018, 290, 12-25. [CrossRef]

30. Xiao, J.; Zhong, S. Synchronization and stability of delayed fractional-order memristive quaternion-valued neural networks with parameter uncertainties. Neurocomputing 2019, 363, 321-338. [CrossRef]

31. Yang, X.; Li, C.; Song, Q.; Chen, J.; Huang, J. Global Mittag-Leffler stability and synchronization analysis of fractional-order quaternion-valued neural networks with linear threshold neurons. Neural Netw. 2018, 105, 88-103. [CrossRef]

32. Qi, X.; Bao, H.; Cao, J. Exponential input-to-state stability of quaternion-valued neural networks with time delay. Appl. Mathe. Comput. 2019, 358, 382-393. [CrossRef]

33. Tu, Z.; Yang, X.; Wang, L.; Ding, N. Stability and stabilization of quaternion-valued neural networks with uncertain time-delayed impulses: Direct quaternion method. Phys. A Stat. Mech. Appl. 2019, 535, 122358. [CrossRef]

34. Li, C.; Zhang, F. A survey on the stability of fractional differential equations. Eur. Phys. J. Spec. Top. 2011, 193, 27-47. [CrossRef]

35. Filippov, A.F. Differential Equations with Discontinuous Righthand Sides; Kluwer Academic: Boston, MA, USA, 1988.

36. Magin, R. Fractional calculus models of complex dynamics in biological tissues. Comput. Math. Appl. 2010, 59, 1586-1593. [CrossRef]

37. Pratap, A.; Raja, R.; Cao, J.; Rajchakit, G.; Lim, C.P. Global robust synchronization of fractional order complex-valued neural networks with mixed time-varying delays and impulses. Int. J. Control Autom. Syst. $2019,17,509-520$. 
38. Xiao, J.; Wen, S.; Yang, X.; Zhong, S. New approach to global Mittag-Leffler synchronization problem of fractional-order quaternion-valued BAM neural networks based on a new inequality. Neural Netw. 2020, 122, 320-337. [CrossRef]

39. Pratap, A.; Raja, R.; Cao, J.; Rihan, F.A.; Seadawy, A.R. Quasi-pinning synchronization and stabilization of fractional-order BAM neural networks with delays and discontinuous neuron activations. Chaos Soliton Fract. 2019, 2019, 109491. [CrossRef]

40. Pratap, A.; Raja, R.; Alzabut, J.; Dianavinnarasi, J.; Cao, J.; Rajchakit, G. Finite-time Mittag-Leffler stability of fractional-order quaternion-valued memristive neural networks with impulses. Neural Process. Lett. 2019. [CrossRef]

41. Fan, Y.; Huang, X.; Wang, Z.; Xia, J.; Li, Y. Global Mittag-Leffler synchronization of delayed fractional-order memristive neural networks. Adv. Differ. Equ. 2018, 2018, 338. [CrossRef]

42. Chang, W.; Zhu, S.; Li, J.; Sun, K. Global Mittag-Leffler stabilization of fractional-order complex-valued memristive neural networks. Appl. Math. Comput. 2018, 338, 346-362. [CrossRef]

(C) 2020 by the authors. Licensee MDPI, Basel, Switzerland. This article is an open access article distributed under the terms and conditions of the Creative Commons Attribution (CC BY) license (http:/ / creativecommons.org/licenses/by/4.0/). 